Distributions of 14 Elements on 60 Selected Absorbers from Truo Simulant Solutions (Acid-Dissolved Sludge and Alkaline Supernate) for Hanford HLW Tank 102-SY

S. Fredric Marsh

Zita V.Svitra

Scott M. Borven 


\section{CONTENTS}

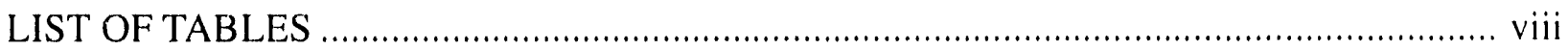

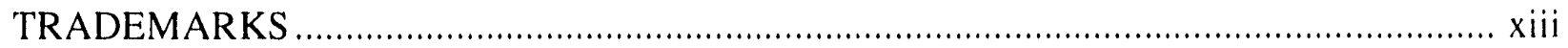

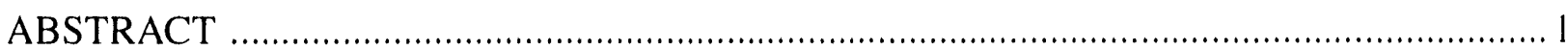

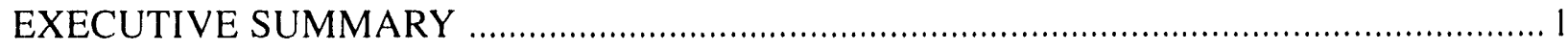

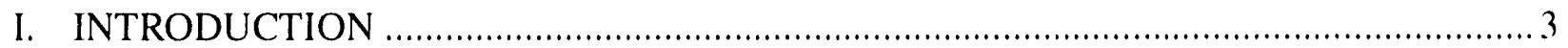

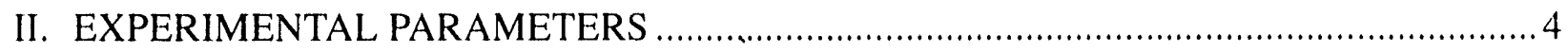

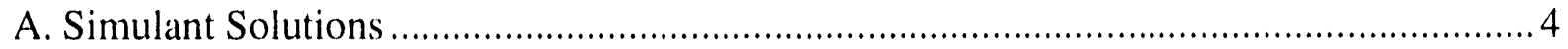

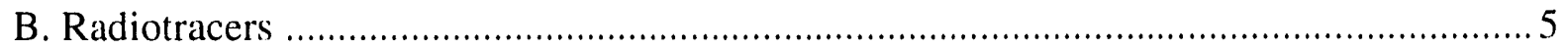

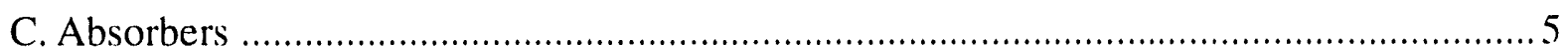

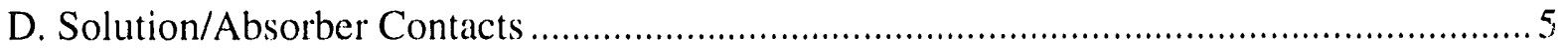

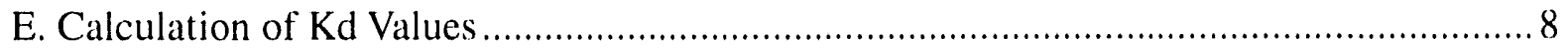

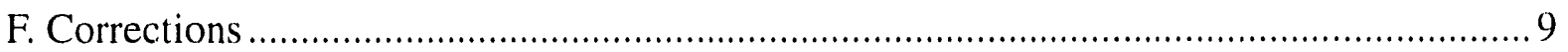

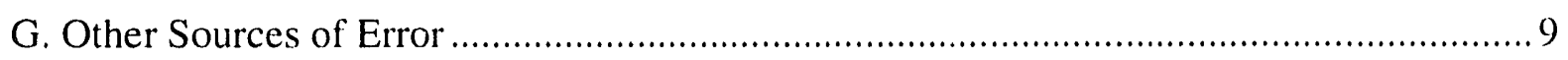

H. Data Transfer and Processing ................................................................................. 10

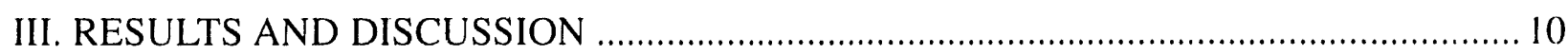

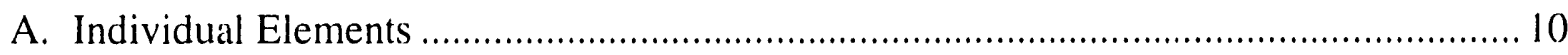

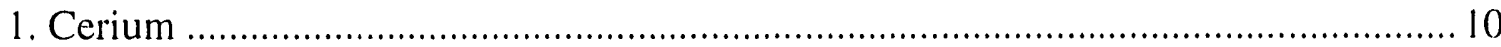

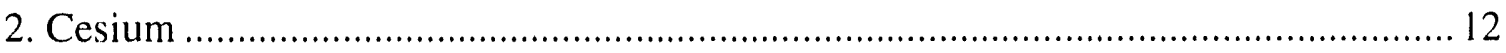

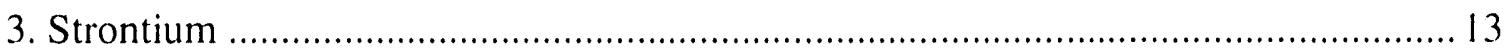

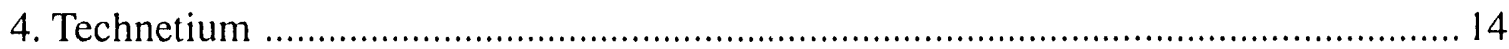

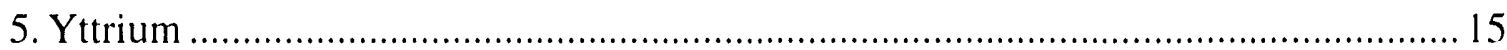

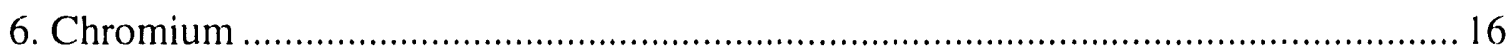

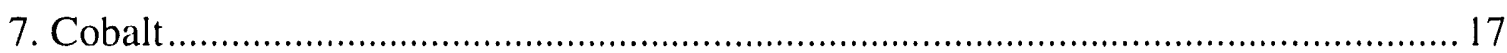

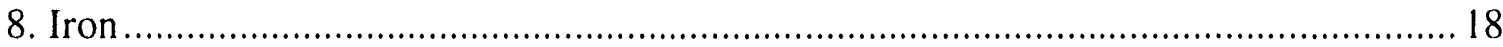

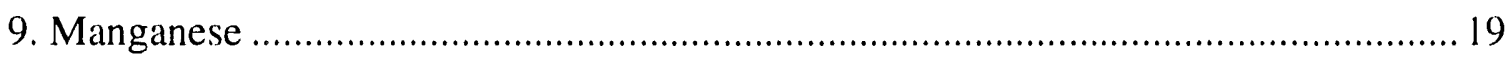

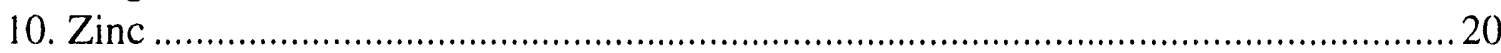

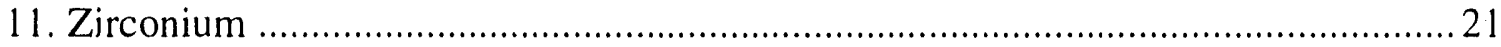

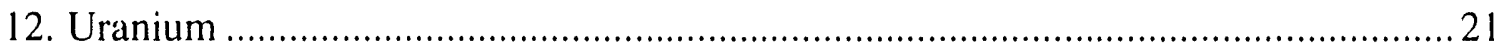

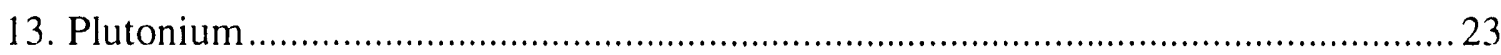

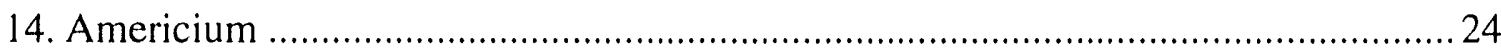

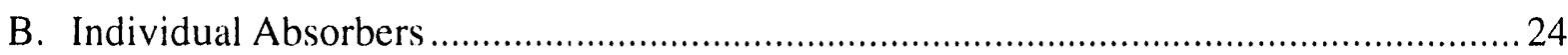

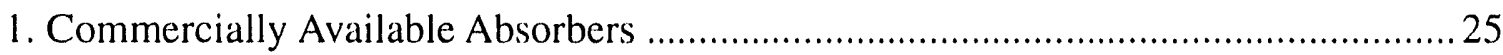

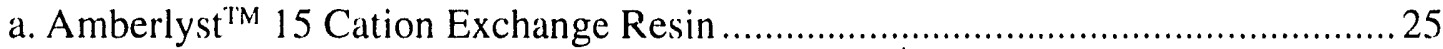

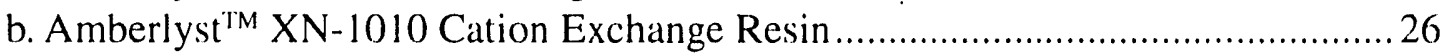

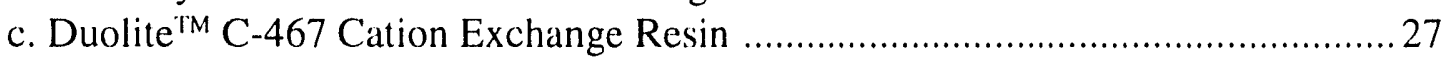

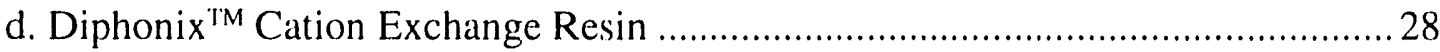

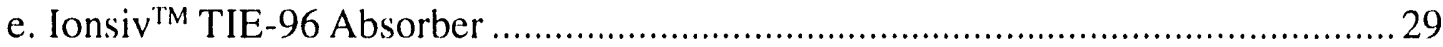

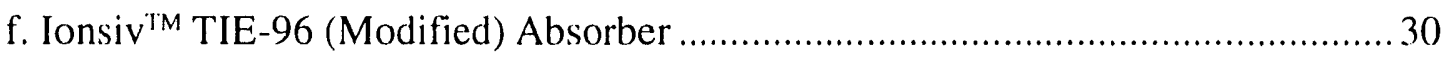

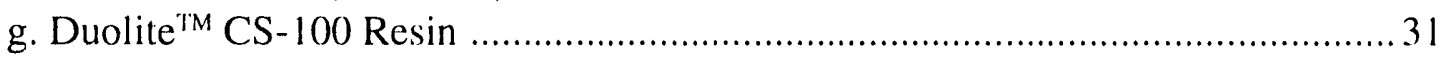




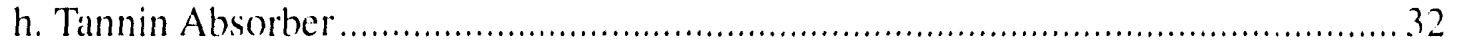

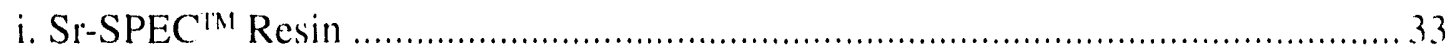

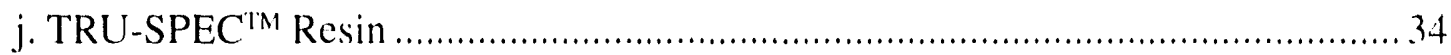

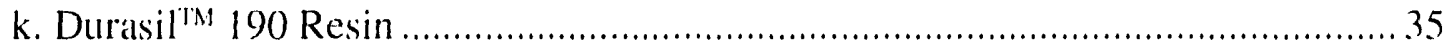

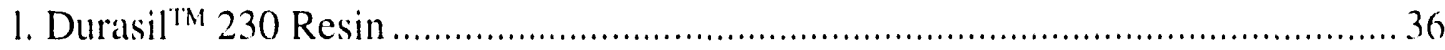

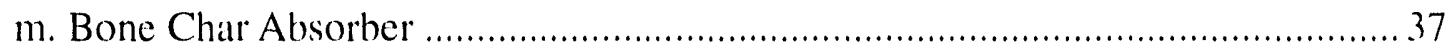

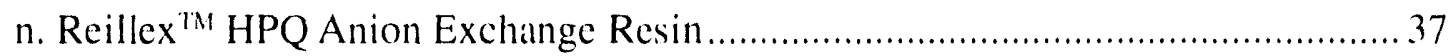

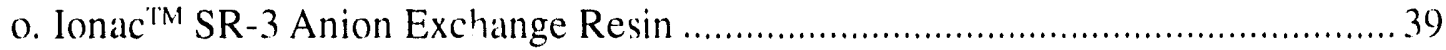

p. Purolite ${ }^{\mathrm{MM}}$ A-520-E Anion Exchange Resin ................................................. 40

q. Ionac ${ }^{\mathrm{TM}}$ SR-6 Anion Exchange Resin ................................................. 41

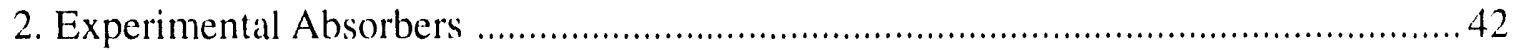

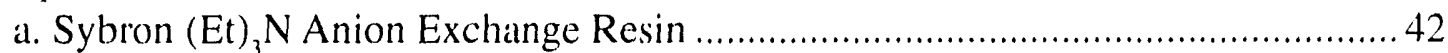

b. Sybron (Pr) $)_{3} \mathrm{~N}$ Anion Exchange Resin ................................................. 43

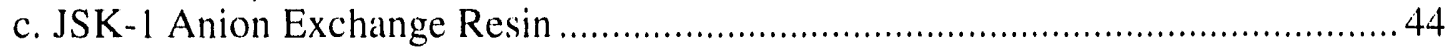

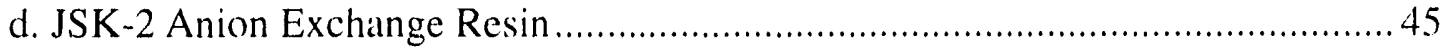

e. JSK-3 Anion Exchange Resin ...................................................................... 46

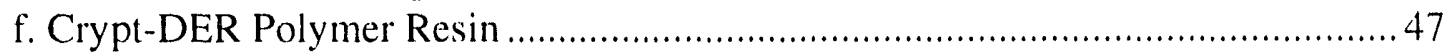

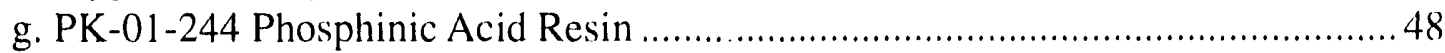

h. SRS Formaldehyde/Resorcinol Resin ................................................. 49

3. Polyacrylonitrile (PAN) Composite Absorbers ............................................5)

a. TiO-PAN Titanium Dioxide Composite .................................................... 50

b. NiFC-PAN Nickel Ferrocyanide Composite ....................................................... 5 I

c. MgO-PAN Magnesium Oxide Composite ......................................................... 52

d. MnO-PAN Manganese Dioxide Composite ........................................................... 53

e. NaY-PAN Sy'nthetic Zeolite Composite ...................................................... 54

f. M315-PAN Synthetic Mordenite Composite .................................................... 55

g. NaTiO-PAN Sodium Titanate Composite .......................................................... 56

h. $\mathrm{Ba}(\mathrm{Ca}) \mathrm{SO}_{4}$-PAN Barium/Calcium Sulfate Composite ...................................... 57

i. AMP-PAN Ammonium Molybdophosphate Composite ................................. 58

4. Phenolsulfonic-Formaldehyde Composite Absorbers ..............................................59

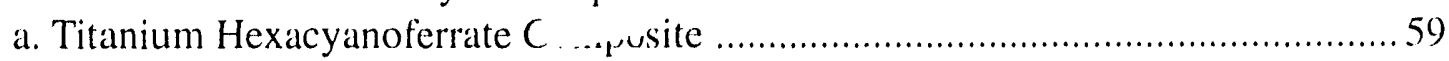

b. Cobalt Hexacyanoferrate Composite ......................................................6 60

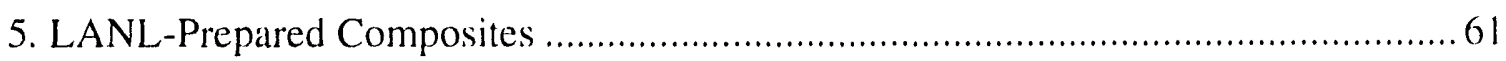

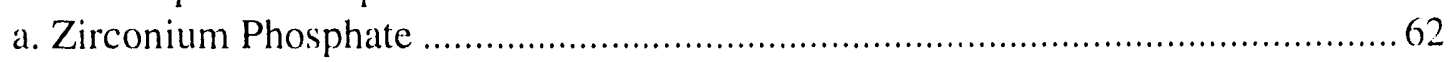

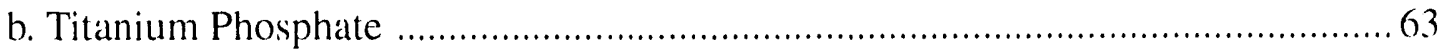

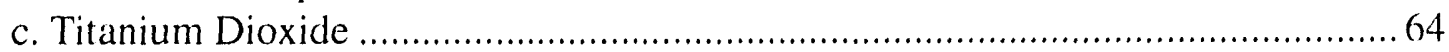

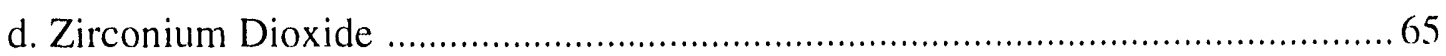

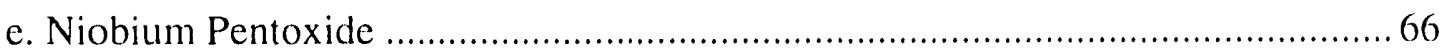

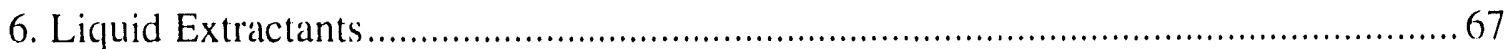

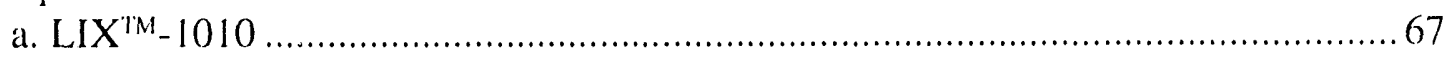

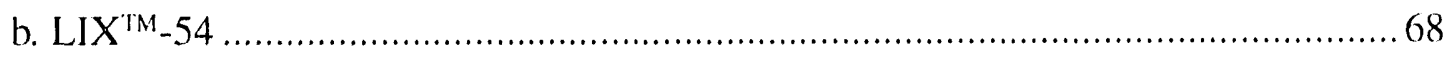




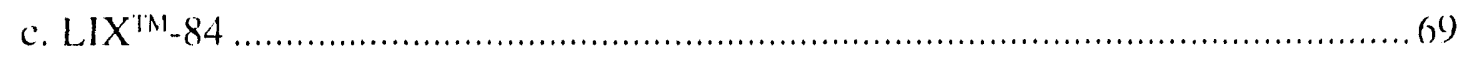

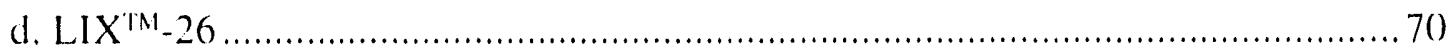

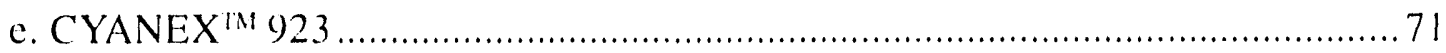

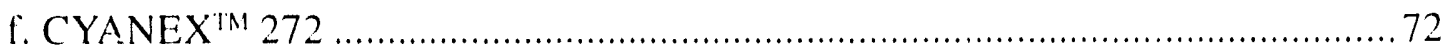

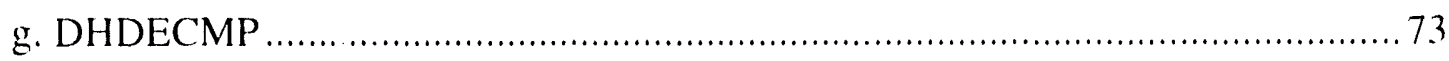

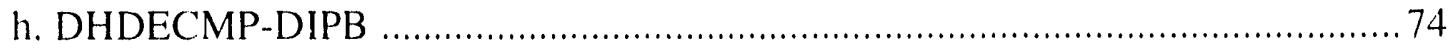

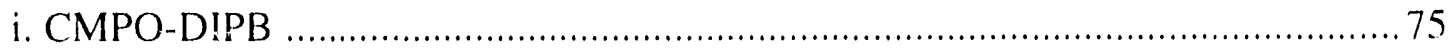

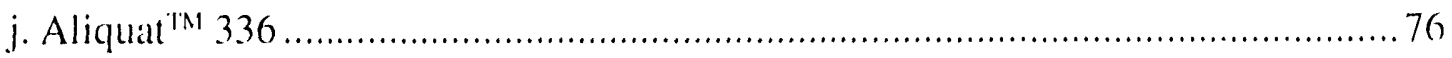

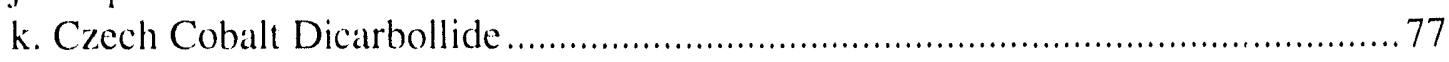

1. INC-Synthesized Cobalt Dicarbollide …………................................................ 78

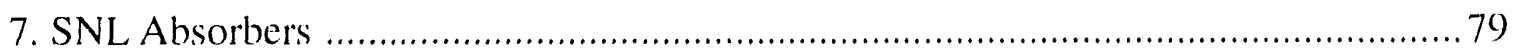

a. SNL/CST35 Crystalline Silico-Titanate …………........................................

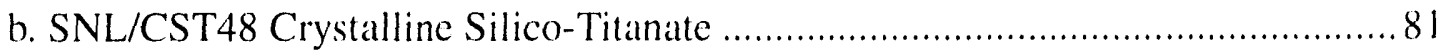

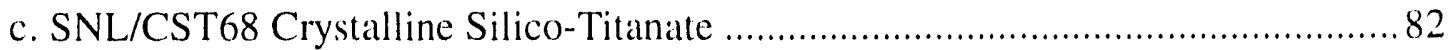

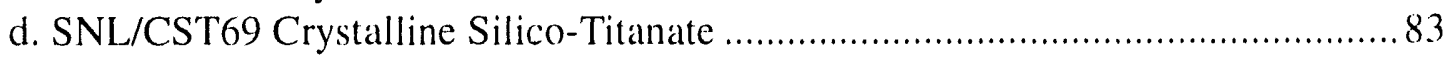

e. SNL/CST84 Crystalline Silico-Titanate …………….......................................... 84

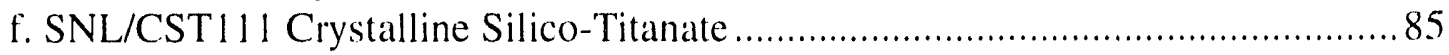

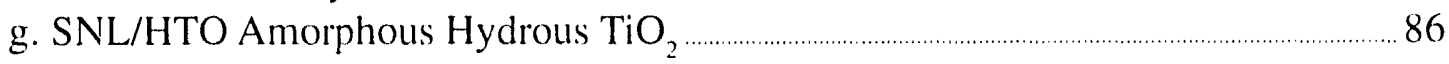

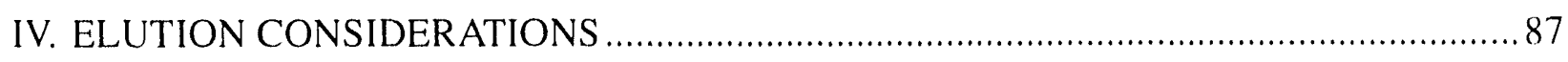

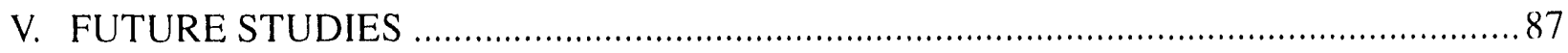

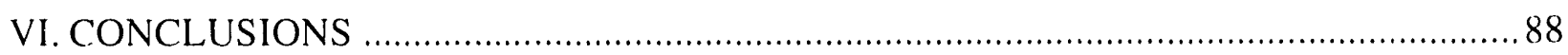

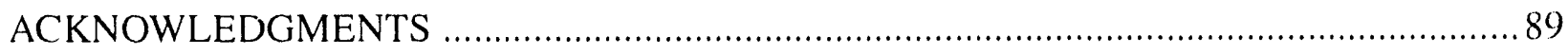

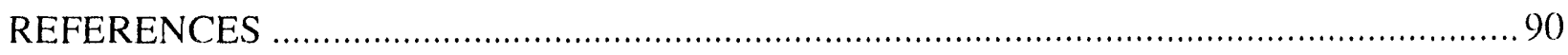

APPENDIX A. Preparation of Acid-Dissolved Sludge Simulant ..............................................92

APPENDIX B. Preparation of Simulated Alkaline Surernate Solution ........................................95 


\section{LIST OF TABLES}

I. Number of Identified Absorbers in Various Kd Categories, for Each of 14 Elements, from Two Simulant Solutions for Hanford HLW Tank 102-SY 2

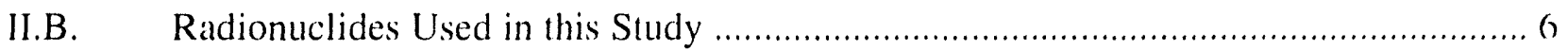

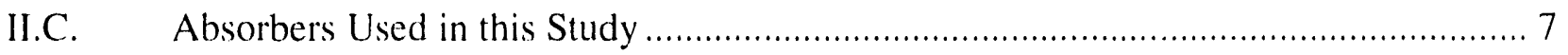

III.A.1. Cerium Distribution Data ............................................................................ i !

III.A.2. Cesium Distribution Data .................................................................. 12

III.A.3. Strontium Distribution Data .................................................................... 1.3

III.A.4. Technetium Distribution Data .......................................................... 14

III.A.5. Yttrium Distribution Data ................................................................................. 15

III.A.6. Chrominm Distribution Data.......................................................................... 16

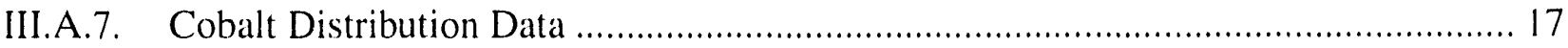

III.A.8. Iron Distribution Data .......................................................................... 18

III.A.9. Manganese Distribution Data ................................................................ 19

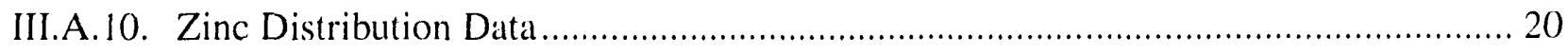

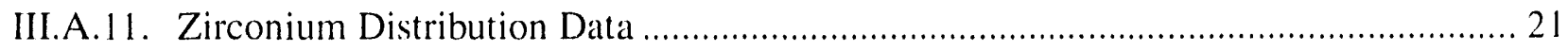

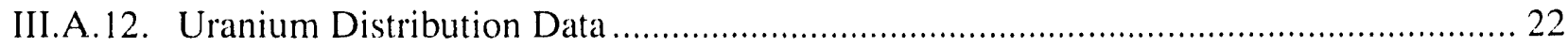

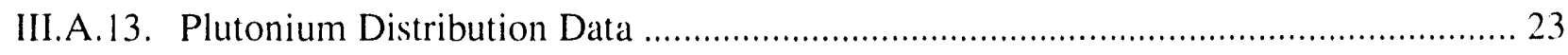

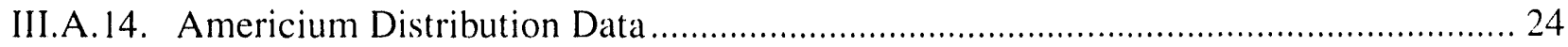

III.B.1.a. Distribution of 14 Elements onto Amberlyst ${ }^{\mathrm{TM}} 15$ Cation Exchange Resin from Two Simulant Solutions for Hanford HLW Tank 102-SY ...................................... 25

III.B.1.b. Distribution of 14 Elements onto Amberlyst ${ }^{\mathrm{TM}} \mathrm{XN}-1010$ Cation Exchange Resin from Two Simulant Solutions for Hanford HLW Tank 102-SY

III.B.1.c. Distribution of 14 Elements onto Duolite ${ }^{\mathrm{TM}} \mathrm{C}-467$ Cation Exchange Resin from Two Simulant Solutions for Hanford HLW Tank 102-SY

III.B.1.d. Distribution of 14 Elements onto Diphonix ${ }^{\mathrm{TM}}$ Cation Exchange Resin from Two Simulant Solutions for Hanford HLW Tank 102-SY

III.B.1.e. Distribution of 14 Elements onto Ionsiv ${ }^{\mathrm{TM}}$ TIE-96 Absorber from Two Simulant Solutions for Hanford HLW Tank 102-SY 
III.B.1.1. Distribution of 14 Elements onto Ionsivin TIE-96 (Modified) Absorber from Two Simulant Solutions for Hanford HLW Tank $102 \mathrm{SY}$

III.B.1.g. Distribution of 14 Elements onto Duolite ${ }^{\mathrm{TM}}$ CS-100 Cation Exchange Resin from Two Simulant Solutions for Hanford HLW Tank 102-SY2

III.B.1.h. Distribution of 14 Elements onto Tannin Absorber from Two Simulant Solutions for Hanford HLW Tank 102-SY

III.B.1.i. Distribution of 14 Elements onto Sr-Spec ${ }^{\mathrm{TM}}$ Resin from Two Simulant Solutions for Hanford HLW Tank 102-SY

III.B.1.j. Distribution of 14 Elements onto TRU-Spec ${ }^{\text {TM }}$ Resin from Two Simulant Solutions for Hanford HLW Tank 102-SY

III.B.1.k. Distribution of 14 Elements onto Durasil ${ }^{\mathrm{TM}} 190$ Resin from Two Simulant Solutions for Hanford HLW Tank 102-SY

III.B.1.1. Distribution of 14 Elements onto Durasil ${ }^{\mathrm{TM}} 230$ Resin from Two Simulant Solutions for Hanford HLW Tank 102-SY 36

III.B.1.m. Distribution of 14 Elements onto Bone Char Absorber from Two Simulant Solutions for Hanford HLW Tank 102-SY

III.B.1.n. Distribution of 14 Elements onto Reillex ${ }^{\mathrm{TM}}$ HPQ Anion Exchange Resin from Two Simulant Solutions for Hanford HLW Tank 102-SY 38

III.B.1.o. Distribution of 14 Elements onto Ionac ${ }^{\mathrm{TM}}$ SR-3 Anion Exchange Resin from Two Simulant Solutions for Hanford HLW Tank 102-SY

III.B.1.p. Distribution of 14 Elements onto Purolite ${ }^{\mathrm{TM}}$ A-520-E Anion Exchange Resin from Two Simulant Solutions for Hanford HLW Tank 102-SY

III.B.1.q. Distribution of 14 Elements onto Ionac ${ }^{\mathrm{TM}}$ SR-6 Anion Exchange Resin from Two Simulant Solutions for Hanford HLW Tank 102-SY

III.B.2.a. Distribution of 14 Elements onto Sybron (Et) ${ }_{3}$ Anion Exchange Resin from Two Simulant Solutions for Hanford HLW Tank 102-SY

III.B.2.b. Distribution of 14 Elements onto Sybron ( $\operatorname{Pr})_{3} \mathrm{~N}$ Anion Exchange Resin from Two Simulant Solutions for Hanford HLW Tank 102-SY

III.B.2.c. Distribution of 14 Elements onto JSK-1 Experimental Anion Exchange Resin from Two Simulant Solutions for Hanford HLW Tank 102-SY

III.B.2.d. Distribution of 14 Elements" onto JSK-2 Experimental Anion Exchange Resin from Two Simulant Solutions for Hanford HLW Tank 102-SY

III.B.2.e. Distribution of 14 Elements onto JSK-3 Experimental Anion Exchange Resin from Two Simulant Solutions for Hanford HLW Tank 102-SY 
III.B.2.f. Distribution of 14 Elements onto Crypt-DER Polymer Resin from Two Simulant Solutions for Hanford HLW Tank 102-SY

III.B.2.g. Distribution of 14 Elements onto PK-()1-244 Resin from Two Simulant Solutions for Hanford HLW Tank 102-SY 48

III.B.2.h. Distribution of 14 Elements onto SRS Formaldehyde/Resorcinol Resin from Two Simulant Solutions for Hanford HLW Tank 102-SY

III.B.3.a. Distribution of 14 Elements onto TiO-PAN Composite Resin from Two Simulant Solutions for Hanford HLW Tank 102-SY 50

III.B.3.b. Distribution of 14 Elements onto NiFC-PAN Composite Resin from Two Simulant Solutions for Hanford HLW Tank 102-SY

III.B.3.c. Distribution of 14 Elements onto MgO-PAN Composite Resin from AcidDissolved Sludge Simulant for Hanford HLW Tank 102-SY 52

III.B.3.d. Distribution of 14 Elements onto MnO-PAN Composite Resin from Two Simulant Solutions for Hanford HLW Tank 102-SY 53

III.B.3.e. Distribution of 14 Elements onto NaY-PAN Composite Resin from Two Simulant Solutions for Hanford HLW Tank 102-SY

III.B.3.f. Distribution of 14 Elements onto M315-PAN Composite Resin from Two Simulant Solutions for Hanford HLW Tank 102-SY 55

III.B.3.g. Distribution of 14 Elements onto NaTiO-PAN Composite Resin from Two Simulant Solutions for Hanford HLW Tank 102-SY 56

III.B.3.h. Distribution of 14 Elements onto $\mathrm{Ba}(\mathrm{Ca}) \mathrm{SO}_{4}-\mathrm{PAN}$ Composite Resin from Two Simulant Solutions for Hanford HLW Tank 102-SY 57

III.B.3.i. Distribution of 14 Elements onto AMP-PAN Composite Resin from Two Simulant Solutions for Hanford HLW Tank 102-SY 58

III.B.4.a. Distribution of 14 Elements onto Titanium Hexacyanoferrate Composite Resin from Two Simulant Solutions for Hanford HLW Tank 102-SY

III.B.4.b. Distribution of 14 Elements onto Cobalt Hexacyanoferrate Composite Resin from Two Simulant Solutions for Hanford HLW Tank 102-SY

III.B.5.a. Distribution of 14 Elements onto Zirconium Phosphate Composite from Two Simulant Solutions for Hanford HLW Tank 102-SY 62

III.B.5.b. Distribution of 14 Elements onto Titanium Phosphate Composite from Two Simulant Solutions for Hanford HLW Tank 102-SY 63 
III.B.5.c. Distribution of 14 Elements onto Titanium Dioxide Composite from 'Two Simulant Solutions for Hanford HLW Tank 1()2-SY

III.B.5.d. Distribution of 14 Elements onto Zirconium Dioxide Composite from Two Simulant Solutions for Hanford HLW Tank 102-SY

111.B.5.e. Distribution of 14 Elements onto Niobium Pentoxide Composite from Two Simulant Solutions for Hanford HLW Tank 102-SY 66

III.B.6.a. Distribution of 14 Elements into LIX ${ }^{\mathrm{TN}}-1010$ from Two Simulant Solutions for Hanford HLW Tank 102-SY

III.B.6.b. Distribution of 14 Elements into LIX ${ }^{\mathrm{TM}}-54$ from Two Simulant Solutions for Hanford HLW Tank 102-SY 68

III.B.6.c. Distribution of 14 Elements into LIX ${ }^{\mathrm{TM}}-84$ from Two Simulant Solutions for Hanford HLW Tank 102-SY

III.B.6.d. Distribution of 14 Elements into LIX ${ }^{\mathrm{TM}}-26$ from Two Simulant Solutions for Hanford HLW Tank 102-SY 70

III.B.6.e. Distribution of 14 Elements into Cyanex ${ }^{\mathrm{TM}} 923$ from Two Simulant Solutions for Hanford HLW Tank 102-SY

III.B.6.f. Distribution of 14 Elements into Cyanex ${ }^{\mathrm{TM}} 272$ from Two Simulant Solutions for Hanford HLW Tank 102-SY

III.B.6.g. Distribution of 14 Elements into DHDECMP from Two Simulant Solutions for Hanford HLW Tank 102-SY

III.B.6.h. Distribution of 14 Elements into DHDECMP-DIPB from Two Simulant Solutions for Hanford HLW Tank 102-SY 74

III.B.6.i. Distribution of 14 Elements into CMPO-DIPB from Two Simulant Solutions for Hanford HLW Tank 102-SY

III.B.6.j. Distribution of 14 Elements into Aliquat ${ }^{\mathrm{TM}} 336$ from Two Simulant Solutions for Hanford HLW Tank 102-SY 76

III.B.6.k. Distribution of 14 Elements into Czech Cobalt Dicarbollide from Two Simulant Solutions for Hanford HLW Tank 102-SY 77

III.B.6.1. Distribution of 14 Elements into INC-Synthesized Cobalt Dicarbollide from Two Simulant Solutions for Hanford HLW Tank 102-SY 78

III.B.7.a. Distribution of 14 Elements onto SNL/CST35 from Two Simulant Solutions for Hanford HLW Tank 102-SY 80

III.B.7.b. Distribution of 14 Elements onto SNL/CST48 from Two Simulant Solutions for Hanford HLW Tank 102-SY 
III.B.7.c. Distribution of 14 Elements onto SNL/CST68 from Two Simulant Solutions for Hanford HLW Tank 102-SY

III.B.7.d. Distribution of 14 Elements onto SNL/CST69 from Two Simulant Solutions for Hanford HLW Tank 102-SY

III.B.7.e. Distribution of 14 Elements onto SNL/CST84 from Two Simulant Solutions for Hanford HLW Tank 102-SY 84

III.B.7.f. Distribution of 14 Elements onto SNL/CST 111 from Two Simulant Solutions for Hanford HLW Tank 102-SY

III.B.7.g. Distribution of 14 Elements onto SNL/HTO from Simulated Alkaline Supernate Solution for Hanford HLW Tank 102-SY 86

A. Composition of Acid-Dissolved Sludge Simulant 92

B. Composition of Simulated Alkaline Supernate Solution 95 


\section{TRADEMARKS}

Aliquat is a registered trademark of the Henkel Corporation, Tucson, AZ,

Tel. 602-622-8891.

Amberlyst is a registered trademark of Rohm \& Haas, Philadelphia, PA,

Tel.215-592-3000.

Cyanex is a registered trademark of American Cyanamid Company, Wayne, NJ, Tel. 800-438-5615

Duolite is a registered trademark of Rohm \& Haas, Philadelphia, PA,

Tel. 215-592-3000.

Durasil is a registered trademark of the Duratek Corporation, Columbia, MD, Tel. 410-312-5100.

EXCEL is a registered trademark of Microsoft Corporation, Redmond, WA, Tel. 800-426-9400.

Ionac is a registered trademark of Sybron Chemicals, Birmingham, NJ, Tel. 609-893-1100.

Ionsiv is a registered trademark of UOP Molecular Sieves, Moorestown, NJ,

Tel. 609-778-6200.

LIX is a registered trademark of the Henkel Corporation, Tucson, AZ, Tel. 602-622-8891.

Purolite is a registered trademark of the Purolite Company, Bala Cynwyd, PA, Tel. 215-668-9090.

Sr-Spec is a registered trademark of EIChroM Industries, Darien, IL, Tel. 800-424-9300.

TRU-Spec is a registered trademark of EIChroM Industries, Darien, IL, Tel. 800-424-9300. 


\title{
Distributions of 14 Elements on 60 Selected Absorbers \\ from Two Simulant Solutions (Acid-Dissolved Sludge and Alkaline \\ Supernate) for Hanford HLW Tank 102-SY
}

by

\author{
S. Fredric Marsh, Zita V. Svitra, and Scott M. Bowen
}

\begin{abstract}
Sixty commercially available or experimental absorber materials were evaluated for partitioning high-level radioactive waste. These absorbers included cation and anion exchange resins, inorganic exchangers, composite absorbers, and a series of liquid extractants sorbed on porous support-beads. The distributions of 14 elements onto each absorber were measured from simulated solutions that represent acid-dissolved sludge and alkaline supernate solutions from Hanford high-level waste (HLW) Tank 102-SY. The selected elements, which represent fission products (Ce, Cs, Sr, Tc, and $\mathrm{Y}$ ); actinides (U, $\mathrm{Pu}$, and $\mathrm{Am}$ ); and matrix elements ( $\mathrm{Cr}, \mathrm{Co}$, $\mathrm{Fe}, \mathrm{Mn}, \mathrm{Zn}$, and $\mathrm{Zr}$ ), were traced by radionuclides and assayed by gamma spectrometry. Distribution coefficients for each of the 1680 element/absorber/solution combinations were measured for $\mathrm{dy}$ namic contact periods of $30 \mathrm{~min}, 2 \mathrm{~h}$, and $6 \mathrm{~h}$ to provide sorption kinetics information for the specified elements from these complex media. More than 5000 measured distribution coefficients are tabulated.
\end{abstract}

\section{EXECUTIVE SUMMARY}

Reliable partitioning agents and technologies are needed to remediate the large quantities of hazardous waste stored in underground tanks at the Hanford Reservation in Washington State. To address this need, we have measured the distribution of 14 elements on 60 different absorbers, from simulants that represent acid-dissolved sludge and alkaline supernate solutions from Hanford high-level waste (HLW) Tank 102-SY.

This screening study, which is intended to identify the most promising absorbers and extractants, included mainly absorbers that either are commercially available or appear that they could be produced in commercial quantities at an acceptable cost, within a reasonable time. Distribution coefficients for each element/ absorber/solution combination were measured for dynamic contact periods of $30 \mathrm{~min}, 2 \mathrm{~h}$, and $6 \mathrm{~h}$ to provide information about the sorption kinetics of each system. 
In many cases, the observed behavior of the absorber/element combination was signilicantly different from what had been expected, based on published meisurements from redaltively cleatn solutions, which demonstrates the value of using realistic simulants for such measurements. In some cases, we found inexpensive commercial materials outperform specially products whose costs are orders of magnitude higher.

Table I summarizes how well each of the 14 elements is sorbed, from both acidic and basic solutions, by showing the number of absorbers identified as effective in eatch of the six distribution coefficient $(\mathrm{Kd})$ categories.

Table I. Number of Identified Absorbers in Various Kd Categories, for Hach of I t likments. from Two Simulant Solutions for Hanford HLW Tank 102-SY

\begin{tabular}{|c|c|c|c|c|c|c|c|}
\hline Medium & Element & $\begin{array}{c}\mathrm{Kd} \\
>1000\end{array}$ & $\begin{array}{c}\text { Kd } \\
300.1000\end{array}$ & $\begin{array}{c}\text { Kd } \\
100-300\end{array}$ & $\begin{array}{c}K d \\
20-100\end{array}$ & $\begin{array}{c}\mathrm{Kd} \\
10.20\end{array}$ & $\begin{array}{c}\mathrm{Kd} \\
5.11\end{array}$ \\
\hline Acidic & $\mathrm{Ce}$ & 0 & 0 & 0 & 5 & 1 & 1 \\
\hline Dissolved & Cs & 7 & 0 & () & 11 & 1 & 4 \\
\hline \multirow[t]{12}{*}{ Sludge } & $\mathrm{Sr}$ & 0 & 0 & 0 & () & () & () \\
\hline & $\mathrm{Tc}$ & 0 & 0 & 1 & 11 & 2 & 5 \\
\hline & Y & 0 & 0 & 0 & 0 & () & () \\
\hline & $\mathrm{Cr}$ & 0 & 0 & 0 & 0 & 0 & () \\
\hline & $\mathrm{Co}$ & 0 & 0 & 0 & 0 & 1 & () \\
\hline & $\mathrm{Fe}$ & 0 & 0 & 0 & 0 & () & () \\
\hline & $\mathrm{Mn}$ & 0 & () & () & 0 & () & () \\
\hline & $\mathrm{Zn}$ & 0 & 0 & 0 & 0 & () & () \\
\hline & $\mathrm{Zr}$ & 0 & 1 & 3 & 10 & 4 & 3 \\
\hline & $\mathrm{U}$ & 0 & 0 & 0 & 6 & 8 & 5 \\
\hline & $\mathrm{Pu}$ & 2 & 5 & 6 & 2 & 8 & 2 \\
\hline & $\mathrm{Am}$ & 0 & 0 & 0 & 0 & () & 6 \\
\hline Alkaline & $\mathrm{Ce}$ & 1 & 3 & 3 & 10 & 3 & 6 \\
\hline \multirow[t]{13}{*}{ Supernate } & Cs & 8 & 1 & 5 & 2 & 4 & 2 \\
\hline & $\mathrm{Sr}$ & 0 & () & () & 0 & 3 & 4 \\
\hline & $\mathrm{Tc}$ & 0 & 7 & 7 & 9 & 3 & 1 \\
\hline & $\mathrm{Y}$ & 1 & 0 & 1 & 9 & () & 5 \\
\hline & $\mathrm{Cr}$ & () & 0 & 0 & 2 & 2 & 5 \\
\hline & Co & 0 & 0 & 0 & 1 & 3 & 4 \\
\hline & $\mathrm{Fe}$ & 0 & 0 & 0 & () & 0 & 3 \\
\hline & $\mathrm{Mn}$ & 0 & 0 & 0 & () & 1 & 5 \\
\hline & $\mathrm{Zn}$ & 0 & 0 & 0 & 5 & 3 & 2 \\
\hline & $\mathrm{Zr}$ & 0 & 0 & 0 & 1 & 4 & 3 \\
\hline & $\mathrm{U}$ & 0 & 1 & 4 & 1.5 & 8 & 8 \\
\hline & $\mathrm{Pu}$ & 0 & 0 & 0 & () & () & 1 \\
\hline & $\mathrm{Am}$ & 0 & 3 & 2 & 13 & 1 & 5 \\
\hline
\end{tabular}




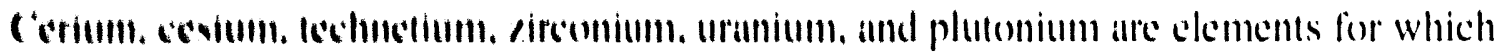

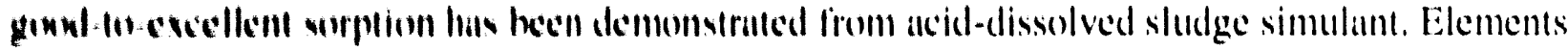
that slum low surption from acidic solution are strontium, yetrium, chromium, cobalt, iron. manganese. sinc: and allicriciuml.

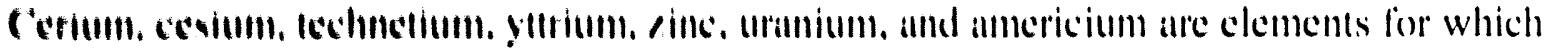

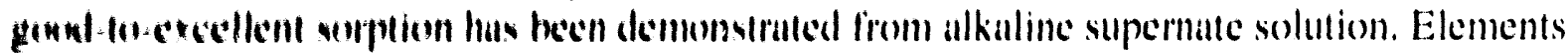
lhat dion pers:lo:marginal sorption from alkalime solution are strontium, chromium, cobalt, iron, mingances, sirconium, and plutumium.

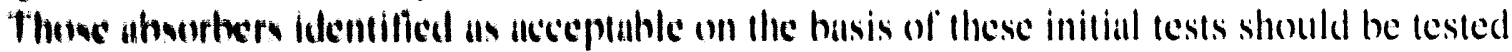
will whe vimulated lank componitions and with actual waste. In the case of elements for which

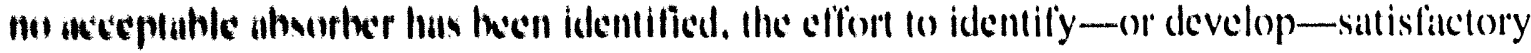
pattillowing agento slowila be accelerited.

Out evperimental revuls indiculc that many existing partitioning agents are suitable for HLW lank rennedialing. If reliahle partitioning agents can be identilied, Hanford HLW lank processing mingli tegin und be emmpleted sooner, and al a lower cost, than would otherwise be possible. We Inefefore feel that the lindings of our study could have a major beneficial impact on decommis- imniny and envirmmentul remediution ciforts ul Hanford and elsewhere within the US Depart-

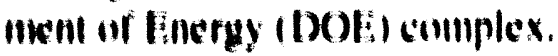

\section{INTR()I)U(CTION}

The Ilanford Renervation in Wishinglon Stale incorporates 177 underground storage tanks Ihat sontan more llan 16.5 million Curies of radionctivity in more than 6.5 million gallons of nave Many different proxesses have heen used al Hanflord for the past lifty years, during which llwe wave hate atedumuluted from nuclear mallerials processing for national defense requiremenl. A. a revull, muny different rengents and waste streams were neutralized and combined in umkefeund lunks, oflen will imullicient concern aboul the computibility of the components twing sorfal The revilling wavles are un extremely complicaled and sometimes unstable mixture if aludges, willeakes, vlurries, and wapermules. Adding significantly to the stored waste problem is Ita leat thal g7 lank, are known, or are suspected, to have leaked.

The It S Depurtment of linergy (DO): is cemmitted to the environmental remediation of

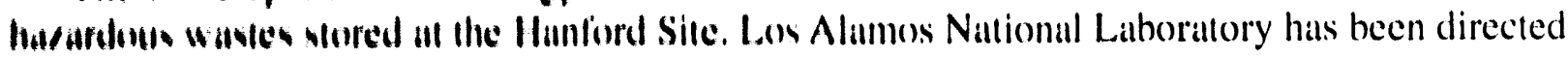
hy IX)I: In nupport llie Ilanliord Tunk Wuste Remediution System (TWRS) mission, which is to "vline, lfeal. and dispone ul all tank waste in a salle. cost-effective, and environmentally sound

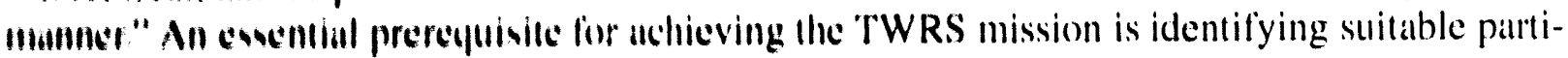
limming agenlo and lechmollogles lo mccomplish this challenging goal.

loin enchunge is a partilioning lechonlongy that has been extensively used in the nuclear

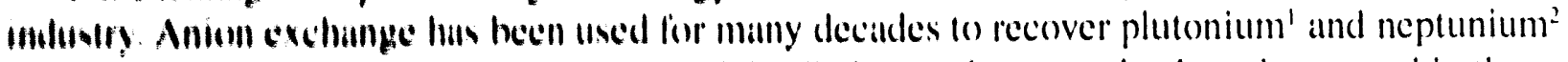
Ifilli a wale variely ul impure muclear muleriuls. Catton exchangers also have been used in the mielear induslry. ' hut generally are comsidered lo be less selective than anion exchange resins.

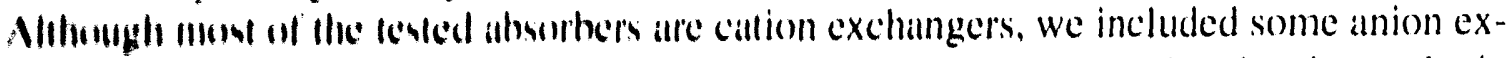
- hange rewill allil a veries of liyuid ion exchangers (sorbed on porous carbon beads) to obtain

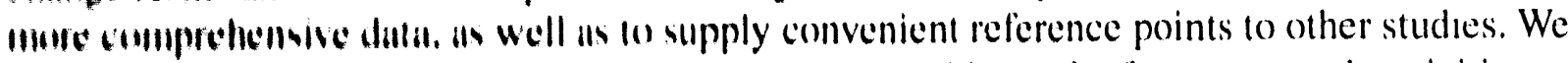

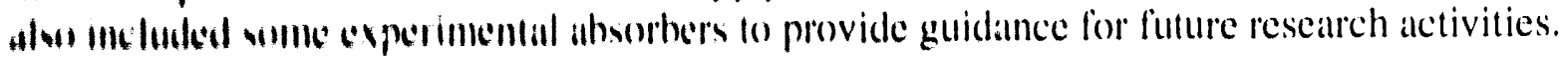


Some of the recently developed specialty products included in our study are reported to offer high selectivity for specific ions; however, few of these absorbers have been evaluated with media approaching the complexity of Hanford high-level waste (HLW) solutions. Nor have any other investigators, to our knowledge, measured the distribution of as many elements (14) onto so many different absorber materials (60).

Our objective was to evaluate a large number of potentially useful absorber materials, including many not previously studied, from realistic simulants of acid-dissolved sludge and alkaline supernate solutions from the Hanford HLW Tank 102-SY. We recognize that no simulant will exactly represent the contents of any HLW tank. Nevertheless, we have used the best information available in our effort to prepare realistic simulants. The most promising absorbers eventually must be tested with actual waste; however, because actual waste samples are expensive and difficult to obtain, we suggest that the preliminary screening of promising candidate absorbers precede tests with actual waste.

Although we include comments and observations about our experimental data, we intentionally do not recommend which absorbers should be used. Our purpose is to identify options from which reliable flowsheets can be developed. The preferred partitioning options will depend heavily on the particular objectives of the flowsheet. Although our study initially was intended to support the Los Alamos flowsheet development program for Hanford Tank 102-SY, ${ }^{4}$ many of our experimental findings are applicable to other flowsheet proposals, such as the so-called "Clean Option." "5ecisions about whether the targeted elements should be recovered individually, or grouped for subsequent disposal, are properly left to the flowsheet designers. Whichever flowsheet is adopted, however, we expect that our experimental data could allow HLW tank processing to begin and be completed sooner, and at a lower cost, than would otherwise be possible.

\section{EXPERIMENTAL PARAMETERS}

\section{A. Simulant Solutions}

The compositions of the two simulant solutions for Hanford HLW Tank 102-SY were based on consultation with and recommendations from Westinghouse Hanford Company (WHC) and Battelle Pacific Northwest Laboratories (PNL) personnel. ${ }^{6}$ The sludge composition was based on the assumption that soluble components had been leached out during thorough mixing with the supernate solution in Tank 102-SY. All our assumptions about tank contents were checked against composition data obtained by reconstruction, based on all available records for transfers into and out of each tank during its entire operating history. ${ }^{7}$

The sludge components that are expected to dissolve during sludge-washing operations, based on PNL sludge-washing studies, ${ }^{8}$ were subtracted from our best estimate of the initial sludge composition and added to our best estimate of the supernate composition. We then dissolved, in a minimum quantity of nitric acid, those components assumed to remain in the sludge. The simulated acid-dissolved sludge was filtered to remove insoluble residue.

The target compositions, actual compositions, and detailed procedure used to prepare the simulated acid-dissolved sludge for Hanford HLW Tank 102-SY are presented in Appendix A. The target compositions, actual compositions, and detailed procedure used to prepare the simulated alkaline supernate solution for Hanford HLW Tank 102-SY are presented in Appendix B. 
We recognize that these simulant solutions (and the actual waste solutions) could be modified and manipulated in various ways to optimize the performance of specific absorbers or extractants. However, each modification of the waste solution would increase the complexity of the process and the quantity of secondary waste, an increase we seek to avoid.

We intentionally avoided modifying the simulant solutions in any way that could bias our study in favor of specific partitioning agents. Instead, we chose to evaluate all the selected partitioning agents on an equal basis with "as-is" simulants. If adequate partitioning technology for "as-is" waste solution cannot be identified, the feed solution can always be modified as required for specific portions of any selected flowsheet; however, in the interests of process simplification and waste minimization, such modifications should be appiied only when they are essential.

\section{B. Radiotracers}

Because gamma spectrometry was selected as the technique for measuring the distribution of the selected elements, suitable radiotracers were obtained and added to the simulant solutions. The selected tracers and the gamma energies measured are listed in Table II.B. The actinides were isolated and purified in the Los Alamos Plutonium Facility. Chromium-51, ${ }^{141} \mathrm{Ce}$, and ${ }^{60} \mathrm{Co}$ were purchased from DuPont New England Nuclear Products, Boston, MA. All other radioisotopes were obtained from the Nuclear Chemistry and Analysis Group, INC-13, Los Alamos National Laboratory.

To minimize the possibility of interference among gamma rays with similar energies, we divided the 14 radiotracers into two easily resolved groups. Each group of tracers was added to a separate portion of each simulant solution. Each of the four resulting solutions was evaluated with each of the 60 absorbers for dynamic contact periods of $30 \mathrm{~min}, 2 \mathrm{~h}$ and $6 \mathrm{~h}$.

\section{Absorbers}

The 60 absorbers evaluated in this study are listed in Table II.C. Details about the supplier or preparation of the absorbers are provided in Section III.B. These absorbers, which encompassed a wide range of particle sizes and porosities, were air-dried and used without further treatment, except where otherwise noted.

\section{Solution/Absorber ontacts}

In most cases, a 250-mg portion of each air-dried absorber was contacted with a measured 6or $8-\mathrm{ml}$ volume of each solution in a specially modified $20-\mathrm{ml}$ disposable hypodermic syringe. We modified these syringes by inserting cylindrical plugs cut from 1/4"-thick porous Kynar (obtained from Porex Technologies, Fairburn, Georgia) into the tapered tips as filters, which permitted liquid, but not most solid absorber materials, to pass through. A 250-mg portion of each dried absorber was added beforehand to an appropriately labeled syringe.

To begin a set of experiments, we pipetted into a plastic beaker a measured volume of the selected solution, which then was drawn into a syringe through the Kynar filter. After sealing the tip of each syringe with a solid Luer cap, we mixed a set of six syringes, prepared as described, on a 48-rpm tube rotator for dynamic contact periods of $30 \mathrm{~min}, 2 \mathrm{~h}$, and $6 \mathrm{~h}$. At the end of each contact period, approximately $25 \%$ of the total solution volume was expelled through the filtered tip of the syringe into a tared counting vial. The weight of the dispensed solution was determined and recorded for later use in the calculation of distribution coefficient $(\mathrm{Kd})$ values. 
Table II.B. Radionuclides Used in this Study

\begin{tabular}{lcc}
\hline Nuclide & Gamma energy, MeV & Branching, \% \\
\hline${ }^{241} \mathrm{Am}$ & .0595 & 35.9 \\
${ }^{2.39} \mathrm{Pu}$ & .1293 & $6.2 \times 10^{-3}$ \\
${ }^{238} \mathrm{Pu}{ }^{\mathrm{a}}$ & .1 .53 & $1.0 \times 10^{-3}$ \\
${ }^{141} \mathrm{Ce}$ & .145 & 48 \\
${ }^{9.5 \mathrm{~m}} \mathrm{Tc}$ & .204 & 100 \\
${ }^{2.37} \mathrm{U}$ & .208 & 23.3 \\
${ }^{51} \mathrm{Cr}$ & .320 & 10 \\
${ }^{88} \mathrm{Zr}$ & .394 & 97 \\
${ }^{85} \mathrm{Sr}$ & .514 & 100 \\
${ }^{1.37} \mathrm{Cs}$ & .662 & 85 \\
${ }^{54} \mathrm{Mn}$ & .835 & 100 \\
${ }^{88} \mathrm{Y}$ & .898 & 92 \\
${ }^{59} \mathrm{Fe}$ & 1.099 & 56 \\
${ }^{65} \mathrm{Zn}$ & 1.115 & 51 \\
${ }^{60} \mathrm{Co}$ & 1.173 & 100 \\
\hline
\end{tabular}

i ${ }^{238} \mathrm{Pu}$ was used as the tracer in all alkaline solutions and in a few of the acidic solutions. When ${ }^{238} \mathrm{Pu}$ was used, the plutonium concentration was $60 \mathrm{mg} / \mathrm{L}$, whereas in the acidic solutions that contained ${ }^{239} \mathrm{Pu}$, the plutonium concentration was $3 \mathrm{~g} / \mathrm{L}$.

These filtered syringes were unsuitable for the Sandia National Laboratories (SNL) crystalline silico-titanate (CST) absorbers because the particle size of the CSTs was much smaller than the pores in the Kynar filters. For these very finely divided materials, we contacted each absorber/solution combination in a $20-\mathrm{ml}$ centrifuge tube, rather than a syringe. At the end of each contact period, the tubes were centrifuged at high speed for $10 \mathrm{~min}$, after which $1.6 \mathrm{ml}$ of solution was transferred into a smaller centrifuge tube, which also was centrifuged at high speed for $10 \mathrm{~min}$.

Finally, approximately $1.5 \mathrm{ml}$ of solution was transferred from the second centrifuge tube into a $10-\mathrm{ml}$ hypodermic syringe having a 0.45 -micron filter attached to the Luer-lock tip. The twicecentrifuged solution was expelled through this submicron filter into a tared counting vial, after which the solution weight was determined and recorded, as was done with the other solutions.

The unusually large variation in Kd values obtained with the CST absorbers indicates that, in spite of our efforts to exclude them, submicron particles sometimes contaminated the solution taken for gamma spectrometric assay. This was a particular problem for cesium, which is very strongly sorbed on CSTs. Even a few particles on which cesium activity is strongly sorbed could introduce a significant amount of activity into a solution that otherwise would be essentially free 
Table II.C. Absorbers Used in this Study

\section{COMMERCIALLY}

AVAILABLE ABSORBERS

EXPERIMENTAL ABSORBERS

COMPOSITE ABSORBERS

Czech Republic

(polyacrylonitrile matrix)
Amberlyst ${ }^{\mathrm{TM}} 15$ cation exchange resin (sulfonic acid)

Amberlyst ${ }^{\mathrm{TM}} \mathrm{XN}-1010$ cation exchange resin (sul-

fonic acid)

Duolite ${ }^{\mathrm{TM}} \mathrm{C}-467$ cation exchange resin (phosphonic acid)

Diphonix ${ }^{\mathrm{TM}}$ cation exchange resin (polyfunctional)

Ionsiv ${ }^{\mathrm{TM}} \mathrm{TIE}-96\left(\mathrm{TiO}_{2}\right.$-loaded zeolite)

Ionsiv ${ }^{\mathrm{TM}} \mathrm{TIE}-96$, modified ( $\mathrm{TiO}_{2}$-loaded zeolite)

Duolite ${ }^{\mathrm{TM}} \mathrm{CS}-100$ resin

Tannin (Mitsubishi)

Sr-Spec ${ }^{\mathrm{TM}}$ resin (EIChrom)

TRU-Spec ${ }^{\text {TM }}$ resin (EIChrom)

Durasil' 190 resin

Durasil $^{\mathrm{TM}} 230$ resin

Bune char

Reillex ${ }^{\mathrm{TM}} \mathrm{HPQ}$ anion exchange resin

Ionac ${ }^{\mathrm{TM}}$ SR-3 anion exchange resin

Purolite ${ }^{\mathrm{TM}} \mathrm{A}-520-\mathrm{E}(\mathrm{Et}){ }_{3} \mathrm{~N}$ anion exchange resin

Ionac $^{\mathrm{TM}} \mathrm{SR}-6$ anion exchange resin

Sybron $^{\mathrm{TM}}(\mathrm{Et})_{3} \mathrm{~N}$ anion exchange resin

Sybron $^{\mathrm{TM}}(\mathrm{Pr})_{3} \mathrm{~N}$ anion exchange resin

JSK-2 anion exchange resin

JSK- 3 anion exchange resin

JSK-4 anion exchange resin

Crypt-DER polymer resin

PK-01-244 phosphinic acid resin

Savannah River formaldehyde/resorcinol resin

TiO-PAN titanium dioxide

NiFC-PAN nickel ferrocyanide

MgO-PAN magnesium oxide

MnO-PAN manganese dioxide

NaY-PAN synthetic zeolite

M315-PAN synthetic mordenite

NaTiO-PAN sodium titanate

$\mathrm{Ba}(\mathrm{Ca}) \mathrm{SO}_{4}-\mathrm{PAN}$ barium/calcium sulfate

AMP-PAN ammonium molybdophosphate
Poland

(phenolsulfonic-formaldehyde matrix)
Titanium hexacyanoferrate

Cobalt hexacyanoferrate 
Table II.C. (Continued)

\begin{tabular}{ll}
\hline LANL-PREPARED & Zirconium phosphate \\
COMPOSITE ABSORBERS & Titinium phosphate \\
& Titanium dioxide \\
& Zirconium dioxide \\
& Niobium pentoxide
\end{tabular}

LANL-PREPARED EXTRACTANT BEADS
LIX $^{\mathrm{TM}}$ - 1010 (di-2-ethylhexylphosphoric acid)

LIX $^{\mathrm{TM}}-54$ (a beta diketone)

$\mathrm{LIX}^{\mathrm{TM}}-84$ (a ketoxime)

LIX $^{\mathrm{TM}}$-26 (8-hydroxyquinoline)

Cyanex ${ }^{\text {TM }} 923$ (trialkylphosphine oxide)

Cyanex ${ }^{\mathrm{TM}} 272$ (trimethylpentylphosphoric acid)

IJHDECMP

DHDECMP-diisopropylbenzene

CMPO-diisopropylbenzene

Aliquat $^{\text {TM }} 336$

Czech cobalt dicarbollide

INC-synthesized cobalt dicarbollide
SANDIA NATIONAL LABORATORIES ABSORBERS
SNL/CST35 crystalline silico-titanate

SNL/CST48 crystalline silico-titanate

SNL/CST68 crystalline silico-titanate

SNL/CST69 crystalline silico-titanate

SNL/CST84 crystalline silico-titanate

SNL/CST111 crystalline silico-titanate

SNL/HTO amorphous hydrous TiO,

of cesium. However, in all such cases the error would cause us to understate the actual Kds for removing cesium from solution. Our reported Kds for the CST materials may therefore be considered as minimum values.

\section{E. Calculation of Kd Values}

Each portion of post-contact solution was assayed hy gamma spectrometry for the specilic gamma energies of the added radionuclides. The portion of each element sorbed was determined indirectly from the difference in the measured gamma activity of the selected radionuclide before and after contact with the specified absorber. 
Distribution coefficients $(\mathrm{Kd})$ were calculated for each element as

$$
\mathrm{Kd}=\frac{(\text { pre-contact activity })-(\text { post-contact activity })}{\text { post-contact activity }} \frac{\mathrm{ml} \text { solution }}{\mathrm{g} \text { absorber }}
$$

We recognize that uncertainties become larger for very small distribution coefficients, when the numerator represents a small difference between two large numbers, as well as for very large distribution coefficients, when the denominator becomes very small. However, the objective of this screening study was to identify absorbers that sorb specific elements very well or poorly. If it is important to determine very large Kd values with high accuracy, such data can be obtained during follow-up studies, which would be limited to the most promising absorbers identified in this study.

\section{F. Corrections}

Because the weight of the post-contact assay solutions usually differed slightly from that of the pre-contact standard solution, each post-contact solution was first normalized to the same weight basis as the standard. Appropriate corrections also were made in the $\mathrm{Kd}$ calculations to account for the fact that the liquid/solid ratio and the remaining activity of every radionuclide decreased as each sequential assay portion was removed.

The activity of each radionuclide in the post-contact solution was compared with the activity of that same nuclide in the pre-contact standard. Because most absorbers were initially added in air-dried form, they sometimes preferentially absorbed the aqueous solvent, which had the effect of increasing the concentration of the solutes in the smaller solution volume. At times, this resulted in the activity of certain radionuclides being higher in the post-contact solution than in the pre-contact solution. Whenever the post-contact/pre-contact solution activity ratio of a radionuclide exceeded unity, we assigned a value of 1.000 to the ratio having the highest value, and then normalized all other radionuclides in that same portion of solution accordingly.

Corrections also were made for short-lived radionuclides, such as 6.8 -day ${ }^{237} \mathrm{U}$, whose decay during the typical gamma assay period was significant. All $\mathrm{Kd}$ calculations and corrections were done using an EXCEL ${ }^{\mathrm{TM}}$ spreadsheet.

\section{G. Other Sources of Error}

Each combination of element/absorber/solution/contact-time was measured only once. The absence of duplicates is partially offset by the fact that we measured individual Kd values for three consecutive contact periods. An abrupt change in the expected smooth trend of these three data points can indicate an unreliable point.

Poor precision is also indicated by excessive variation within a set of three data points where no trend is evident. For very low or very high sorption, the variation is expected to be high for reasons already discussed. The uncertainty for these worst cases appears to be within a factor of two to three. We estimate the unertainty associated with the best $\mathrm{Kd}$ values to be 10-20 relative percent.

At times, $\mathrm{Kd}$ values are highest for the 30 -min contact and decrease thereafter. Several seenarios could explain these decreasing Kd values. (1) The element in question might sorb rapidly and then be displaced by another slower-sorbing species. (2) Some of the strong complexing anions in the simulants could be participating in slow competitive reactions with the caltions 
being measured. (3) The absorber could be representatively or selectively dissolving, thereby releasing previously sorbed cations. (Slow dissolution of an amorphous phase within the crystalline silico-titanates was suggested by Sandia ivational Laboratories (SNL) personnel to explain decreasing $\mathrm{Kd}$ values of initially sorbed non-cesium cations.) And finally, (4) the decreasing $\mathrm{Kd}$ values could be solely due to experimental error.

Finally, the difficulty in accurately correcting for the complex background under the lowenergy $(59.5-\mathrm{keV})$ gamma peak of ${ }^{2+1} \mathrm{Am}$ resulted in this nuclide having larger-than-normal uncertainties associated with its Kd values.

\section{H. Data Transfer and Processing}

To minimize the need for transcribing large amounts of data, and the human errors that can be introduced during such operations, we automated the data transfer and calculation process. The area of each gamma peak of interest was determined from the raw dala in the multichannel analyzer memory, using Gamanal," which provides a complete qualitative and quantitative analysis of mixtures of radionuclides by computer interpretation of high-resolution gamma spectra. Gamanal determines background, fits and resolves complex peak groupings, determines the energies and absolute intensities of the gamma rays, and calculates the quantities of the source nuclides. All interferences are resolved by a least-squares solution of the matrix of equations for the gamma intensities.

The Gamanal output was electronically imported into an EXCEL ${ }^{\mathrm{TM}}$ spreadsheet, in which all the described calculations and corrections were applied. Finally, all calculated Kds were combined into one master spreadsheet and converted to a database to provide tabulated comparisons of the measured Kds according to element, absorber, and solution type. Because small Kd values have large associated uncertainties, we rounded all values to not more than one place beyond the decimal point.

\section{RESULTS AND DISCUSSION}

\section{A. Individual Elements}

Tables III.A.3 through III.A. 16 list the most promising absorbers for each element, ranked in order of their $\mathrm{Kd}$ values, from acid-dissolved sludge and from alkaline supernate simulant solutions. Partitioning agents that sorb the specified element poorly are generally omitted from these tables. However, those absorbers that have been designated as the baseline technologies for TWRS are always included, even when they perform poorly.

1. Cerium. Aside from the JSK experimental anion exchange resins, Reillex ${ }^{\mathrm{TM}}$ HPQ provides the highest sorption of cerium from acidic solution, within $2 \mathrm{~h}$. From basic solution, Cyanex ${ }^{\text {TM }} 923$, a low-cost commercial extractant, outperforms specialty products costing orders of magnitude more. 
Table III.A.1. Cerium Distribution Datta

\begin{tabular}{|c|c|c|c|c|}
\hline Medium & Absorber & 30-min Kd & 2-h Kd & 6-h Kd \\
\hline \multirow{9}{*}{ Acid-Dissolved Sludge } & JSK-1 resin & 28 & 29 & 27 \\
\hline & JSK-2 resin & 27 & 26 & 24 \\
\hline & JSK-3 resin & 23 & 23 & 22 \\
\hline & Reillex ${ }^{\mathrm{TM}} \mathrm{HPQ}$ resin & 14 & 13 & 13 \\
\hline & Purolite ${ }^{\mathrm{TM}} \mathrm{A}-520-\mathrm{E}$ resin & 7.8 & 14 & 21 \\
\hline & Ionac ${ }^{\mathrm{TM}} \mathrm{SR}-3$ resin & 6.6 & 13 & 21 \\
\hline & Crypt-DER resin & 3.0 & 2.9 & 2.8 \\
\hline & Duolite ${ }^{\mathrm{MN}} \mathrm{C}-467$ resin & 2.4 & 1.5 & 2.2 \\
\hline & Amberlyst ${ }^{\mathrm{TM}} 15$ resin & 2.0 & 1.7 & 1.8 \\
\hline \multirow[t]{22}{*}{ Alkaline Supernate } & Cyanex ${ }^{\mathrm{TM}} 923$ & 744 & 988 & 1596 \\
\hline & TRU-Spec ${ }^{\mathrm{TM}}$ resin & 547 & 594 & 644 \\
\hline & SNL/HTO & 265 & 320 & 512 \\
\hline & CMPO-DIPB & 78 & 370 & 499 \\
\hline & Amberlyst ${ }^{\mathrm{TM}} 15$ resin & 42 & 77 & 132 \\
\hline & LIX $^{\mathrm{TM}}-26$ & 32 & 40 & 42 \\
\hline & DHDECMP & 26 & 45 & 51 \\
\hline & Czech MgO-PAN & 26 & 59 & 222 \\
\hline & Czech MnO-PAN & 24 & 25 & 26 \\
\hline & Diphonix $^{\mathrm{TM}}$ resin & 22 & 2.3 & 22 \\
\hline & Bone char & 19 & 33 & 53 \\
\hline & DHDECMP-DIPB & 18 & 28 & 28 \\
\hline & SNL/CSTIII & 17 & 32 & 57 \\
\hline & SRS resorcinol resin & 14 & 26 & 42 \\
\hline & Amberlyst ${ }^{\mathrm{TM}} \mathrm{XN}-1010$ resir & $\sin 13$ & 38 & 114 \\
\hline & Czech AMP-PAN & 12 & 22 & 28 \\
\hline & SNL/CST68 & 8.6 & 10 & 38 \\
\hline & SNL/CST48 & 6.4 & 8.6 & 17 \\
\hline & Duolite ${ }^{\mathrm{NM}} \mathrm{C}-467$ resin & 6.3 & 4.3 & 3.6 \\
\hline & Czech $\mathrm{Ba}(\mathrm{Ca}) \mathrm{SO}_{4}-\mathrm{PAN}$ & 5.4 & 6.1 & 8.6 \\
\hline & Purolite ${ }^{1 \mathrm{M}} \mathrm{A}-520-\mathrm{E}$ resin & 4.8 & 5.3 & 6.1 \\
\hline & Czech NaY-PAN & 4.6 & 4.0) & 3.3 \\
\hline
\end{tabular}


2. Cesium. SNL crystalline silico-titanates outperform all other absorbers from both acidic and basic solution. Caech AMP-PAN composite also performs very well from both acidic and basic solution. Czech NiFC-PAN composite sorbs eesium highly from basic solution, but only moderately from acidic solution. The fact that many of these absorbers sorb cesium strongly from both acidic and basic solution suggests that elution may be difficult.

Table III.A.2. Cesium Distribution Dalta

\begin{tabular}{|c|c|c|c|c|}
\hline Medium & Absorber & 30-min Kd & 2-h Kd & 6-h Kd \\
\hline \multirow[t]{13}{*}{ Acid-Dissolved Sludge } & SNL/CST 35 & 448.5 & 1011 & 3416 \\
\hline & SNL/CST111 & 3420 & 5() 21 & 5305 \\
\hline & SNL/CST69 & 2884 & 2279 & 3252 \\
\hline & SNL/CST84 & 1849 & 8096 & 11840 \\
\hline & Czech AMP-PAN & 1626 & 4073 & 5.504 \\
\hline & SNL/CST48 & 794 & 758 & 1180 \\
\hline & SNL/CST68 & 688 & 2307 & 1049 \\
\hline & Czech M315-PAN & 50 & 64 & 61 \\
\hline & Czech NiFC-PAN & 45 & 49 & 49 \\
\hline & Duracil $^{\mathrm{TN}} 230$ & 11 & 14 & 19 \\
\hline & Ionsiv ${ }^{\mathrm{M}}$ TIE-96 (mod.) & 7.7 & 9.0 & 9.4 \\
\hline & Ionsiv ${ }^{\mathrm{TNI}} \mathrm{TIE}-96$ & 7.6 & 7.9 & 8.3 \\
\hline & Duolite ${ }^{\mathrm{rM}} \mathrm{CS}-100$ resin & 0.5 & 1.1 & 1.3 \\
\hline \multirow[t]{15}{*}{ Alkaline Supernate } & SNL/CST35 & 1.31 .58 & 12421 & 4195 \\
\hline & SNL/CST69 & 8879 & 10844 & 20880 \\
\hline & SNL/CST84 & 5484 & 3953 & 3098 \\
\hline & SNL/CSTI11 & 4938 & 2713 & 1864 \\
\hline & SNL/CST68 & 4072 & 2453 & 722 \\
\hline & SNL/CST48 & 3951 & 2820 & 10.31 \\
\hline & Crech NiFC-PAN & 3.5 .3 .3 & 11954 & 5998 \\
\hline & Crech AMP-PAN & 21.33 & 46.36 & 8275 \\
\hline & Crech M315-PAN & 191 & 227 & 2.31 \\
\hline & Durasili ${ }^{\mathrm{TM}} 230$ & 78 & 1.3 .5 & 148 \\
\hline & SRS resorcinol resin & 75 & 8.5 & 66 \\
\hline & Ionsiv'MI TIE-96 (mod.) & 6.3 & 110 & 139 \\
\hline & Ionsivin TIE-96 & 58 & 97 & 122 \\
\hline & SNL.HTO & 10 & 8.9 & 8.5 \\
\hline & Duolite ${ }^{\mathrm{M} M} \mathrm{CS}-100$ resin & 1.3 & 1.2 & 1.3 \\
\hline
\end{tabular}


3. Strontium. Although Sr-Spec ${ }^{\mathrm{N}}$ resin sorbs strontium better than the other absorbers from both acidic and basic solution, in neither case is the sorption very high. From hasi solution, Amberlyst ${ }^{\mathrm{TN}} 15$ cation exchange resin, whose cost is hundreds of times less than that of $\mathrm{Sr}$ Spec rM resin, performs nearly as well. The poor-to-marginal performance of all the studied absorbers for strontium indicates that better partitioning agents are needed to remove this important fission product.

4. Technetium. Reillex ${ }^{\mathrm{TN}} \mathrm{HPQ}$ resin offers the highest $\mathrm{Kd}$ for technetium from acidic solution and an even higher $\mathrm{Kd}$ from hasic solution. Although Reillex ${ }^{\text {IN }} \mathrm{HPQ}$ resin provides $\mathrm{Kd}$ values of at least 250 from basic solution, two experimental resins and two commercial extractants outperform it. Aliquat ${ }^{\mathrm{TM}} 336$ is especially interesting because it is nearly selective for sorbing technetium from basic solution, but not from acid, which indicates that acid should be an effective stripping agent.

Table III.A.3. Strontium Distribution Data

\begin{tabular}{|c|c|c|c|c|}
\hline Medium & Absorber & 30-min Kd & 2-h Kd & 6-h Kd \\
\hline \multirow[t]{5}{*}{ Acid-Dissolved Sludge } & Sr-Spec ${ }^{\mathrm{IN}}$ resin & 3.0 & 3.1 & 2.9 \\
\hline & Diphonix ${ }^{1 M}$ resin & 2.0 & 1.7 & 1.3 \\
\hline & Ionsivi TIE TIE-96 & 1.9 & 1.0 & 1.5 \\
\hline & Czech $\mathrm{Ba}(\mathrm{Ca}) \mathrm{SO}_{4}-\mathrm{PAN}$ & 1.5 & 1.7 & 1.5 \\
\hline & Czech MgO-PAN & 1.4 & 1.6 & 2.0 \\
\hline \multirow[t]{12}{*}{ Alkaline Supernate } & Sr-Spec ${ }^{\mathrm{TM}}$ resin & 18 & 18 & 17 \\
\hline & Amberlyst ${ }^{\mathrm{TM}} 15$ & 12 & 13 & 13 \\
\hline & Crech $\mathrm{Ba}(\mathrm{Ca}) \mathrm{SO}_{4}-\mathrm{PAN}$ & 7.3 & 8.1 & 12 \\
\hline & Amberlyst ${ }^{\mathrm{TN}} \mathrm{XN}-1010$ & 5.9 & 7.8 & 8.3 \\
\hline & Czech NaTiO-PAN & 4.9 & 5.3 & 5.0 \\
\hline & SRS resorcinol resin & 4.9 & 2.6 & 1.5 \\
\hline & SNL/CST 111 & 4.2 & 4.2 & 3.5 \\
\hline & SNL/CST69 & 3.4 & 2.9 & 6.6 \\
\hline & Crech MgO-PAN & 3.0 & 4.8 & 7.4 \\
\hline & SNL/CST84 & 2.2 & 2.0 & 4.4 \\
\hline & SNL/CST 35 & 1.6 & 1.3 & 3.3 \\
\hline & SNL/HTO & 1.5 & 1.4 & 3.6 \\
\hline
\end{tabular}


Table III.A.4. Technetium Distribution Data

\begin{tabular}{|c|c|c|c|c|}
\hline Medium & Absorber & 30)-min Kd & 2-h Kd & 6-h Kd \\
\hline \multirow[t]{13}{*}{ Acid-Dissolved Sludge } & Reillex ${ }^{\mathrm{TM}} \mathrm{HPQ}$ resin & i6 & 90 & 93 \\
\hline & Ionac ${ }^{\mathrm{TM}} \mathrm{SR}-3$ resin & 32 & 44 & 43 \\
\hline & Purolite ${ }^{\mathrm{TM}} \mathrm{A}-520-\mathrm{E}$ resin & 32 & 57 & 77 \\
\hline & Sybron $^{\mathrm{TM}}(\mathrm{Et})_{3} \mathrm{~N}$ resin & 31 & 51 & 53 \\
\hline & LANL TiO, & 19 & 40 & 57 \\
\hline & LANL $\mathrm{ZrO}_{2}^{-}$ & 17 & 41 & 60 \\
\hline & Duolite $^{\mathrm{TM}} \mathrm{C}-467$ resin & 12 & 12 & 13 \\
\hline & Sybron $^{\mathrm{TM}}(\operatorname{Pr})_{3} \mathrm{~N}$ resin & 11 & 26 & 45 \\
\hline & Tannin & 10 & 39 & 136 \\
\hline & LANL $\mathrm{Nb}_{2} \mathrm{O}_{5}$ & 6.9 & 17 & 31 \\
\hline & Duolite ${ }^{\mathrm{TM}} \mathrm{CS}-100$ resin & 4.8 & 6.3 & 6.4 \\
\hline & Ionac ${ }^{\mathrm{MM}} \mathrm{SR}-6$ resin & 2.1 & 4.6 & 8.4 \\
\hline & SRS resorcinol resin & 2.1 & 4.6 & 11 \\
\hline \multirow[t]{18}{*}{ Alkaline Supernate } & Sybron $^{\mathrm{TN}}(\operatorname{Pr})_{3} \mathrm{~N}$ resin & 401 & 777 & 992 \\
\hline & Cyanex $^{\mathrm{TM}} 923$ & 300 & 551 & 582 \\
\hline & Aliquat $^{\mathrm{TM}} 336$ & 295 & 443 & 572 \\
\hline & Sybron $^{\mathrm{TM}}(\mathrm{Et})_{3} \mathrm{~N}$ resin & 264 & 405 & 440 \\
\hline & Reillex ${ }^{\mathrm{TM}} \mathrm{HPQ}$ resin & 250 & 280 & 274 \\
\hline & Purolite ${ }^{\mathrm{TM}} \mathrm{A}-520-\mathrm{E}$ resin & 205 & 320 & 380 \\
\hline & Ionac ${ }^{\mathrm{TM}}$ SR-6 resin & 151 & 394 & 618 \\
\hline & Ionac ${ }^{\mathrm{TM}} \mathrm{SR}-3$ resin & 121 & 123 & 125 \\
\hline & TRU-Spec ${ }^{\mathrm{TM}}$ resin & 90 & 78 & 74 \\
\hline & LANL $\mathrm{TiO}_{2}$ & 54 & 185 & 290) \\
\hline & LIX $^{\mathrm{TM}}-26$ & 54 & 112 & 190 \\
\hline & Sr-Spec ${ }^{\mathrm{TM}}$ resin & 45 & 42 & 39 \\
\hline & DHDECMP & 43 & 112 & 222 \\
\hline & CMPO-DIPB & 41 & 111 & 196 \\
\hline & Duolite ${ }^{\mathrm{NM}} \mathrm{C}-467 \mathrm{resin}$ & 40 & 42 & 44 \\
\hline & LANL $\mathrm{ZrO}_{2}$ & 35 & 148 & 258 \\
\hline & LANL $\mathrm{Nb}_{2} \mathrm{O}_{9}$ & 28 & 1.34 & 271 \\
\hline & Crypt-DER resin & 25 & 25 & 25 \\
\hline
\end{tabular}


5. Yttrium. None of the absorbers sorbs yttrium well from acidic solution. From hasic solution, however, Cyanex ${ }^{\mathrm{TN}}$ 92.3, a low-cost commercial extractant, sorbs yttrium at least tenfold better than much-costlier alternatives.

Table III.A.5. Yttrium Distribution Data

\begin{tabular}{|c|c|c|c|c|}
\hline Medium & Absorber & nin Kd & 2-h Kd & 6-h Kd \\
\hline \multirow[t]{10}{*}{ Acid-Dissolved Sludge } & Amberlyst ${ }^{\mathrm{M}} 15$ resin & 2.3 & 1.7 & 2.1 \\
\hline & Duolite $\mathrm{CM}-467$ resin & 1.8 & 0.9 & 1.6 \\
\hline & JSK-1 resin & 1.7 & 1.3 & 1.1 \\
\hline & JSK-2 resin & 1.6 & 1.7 & 1.4 \\
\hline & lonac ${ }^{\mathrm{TM}} \mathrm{SR}-3$ resin & 1.5 & 1.7 & 1.4 \\
\hline & Ionsiv ${ }^{1 M}$ TIE-96 & 1.5 & 0.9 & 1.2 \\
\hline & Amberlyst ${ }^{\mathrm{TM}} \mathrm{XN}-1010$ resin & 1.4 & 1.4 & 1.5 \\
\hline & Purolite ${ }^{T M} A-520-E$ resin & 1.4 & 1.1 & 1.1 \\
\hline & Reillex ${ }^{\mathrm{TM}} \mathrm{HPQ}$ resin & 1.2 & 0.5 & 0.8 \\
\hline & Tannin & 1.2 & 1.2 & 0.7 \\
\hline \multirow[t]{11}{*}{ Alkaline Supernate } & Cyanex ${ }^{\mathrm{TM}} 923$ & 078 & 2014 & 2981 \\
\hline & TRU-Spec ${ }^{\mathrm{TM}}$ resin & 98 & 94 & 92 \\
\hline & SNL/HTO & 53 & 59 & 52 \\
\hline & Diphonix ${ }^{\mathrm{TM}}$ resin & 28 & 31 & 31 \\
\hline & Amberlyst ${ }^{\mathrm{TM}} 15$ resin & 27 & 45 & 66 \\
\hline & Czech MgO-PAN & 26 & 59 & 258 \\
\hline & CMPO-DIPB & 15 & 45 & 89 \\
\hline & Czech AMP-PAN & 15 & 27 & 34 \\
\hline & SRS resorcinol resin & 12 & 21 & 36 \\
\hline & Bone char & 11 & 18 & 26 \\
\hline & Amberlyst ${ }^{\mathrm{TM}} \mathrm{XN}-101$ resin & 11 & 26 & 64 \\
\hline
\end{tabular}


6. Chromium. None of the lested absorbers removes chromium adedualdely from andidie solution. Although four absorbers offer double-digit Kol values for 6-h contacels from balsic solution, these Kds are signilicantly lower for shorter contace periods. The porot-lo-margimal performance of all the studied absorbers for chromium indicalles that betler partitioning anemes are needed to remove this important matrix element.

Table III.A.6. Chiomium Distribution Dalla

\begin{tabular}{|c|c|c|c|c|}
\hline Medium & Absorber & 30-min Kd & 2.h Kd & 6.h Kal \\
\hline \multirow[t]{9}{*}{ Acid-Dissolved Sludge } & Crech MgO-PAN & 1.5 & 1.4 & 1.0 \\
\hline & LANL Zar $\left(\mathrm{PO}_{4}\right)_{4}$ & 1.4 & 1.1 & 1.3 \\
\hline & IOnsiv' $1 \mathrm{NI}$ TIE-96 & 1.4 & 0.8 & 1.1 \\
\hline & LANL Ti ${ }_{3}\left(\mathrm{PO}_{4}\right)_{1}$ & 1.2 & 1.1 & 0.8 \\
\hline & Crach $\mathrm{Ba}(\mathrm{Ca}) \mathrm{SO}_{4}-\mathrm{PAN}$ & 1.1 & 0.9 & ().9 \\
\hline & Duolite ${ }^{\mathrm{N}} \mathrm{C}-467$ resin & 1.1 & 0.4 & 1.0 \\
\hline & Diphonix ${ }^{\text {IN }}$ resin & 1.1 & 0.6 & $(1) .8$ \\
\hline & SNL/CSTIII & 1.0 & 1.0 & 1.2 \\
\hline & Amberlyst ${ }^{\mathrm{N}} 1.5$ resin & 1.0 & 0.7 & (1).9) \\
\hline \multirow[t]{15}{*}{ Alkaline Supernate } & SNL/HTO & 5.9 & 7.8 & 21 \\
\hline & Amberlyst ${ }^{\mathrm{IM}} 1.5 \mathrm{resin}$ & 4.2 & 7.9 & 1.4 \\
\hline & Crech MgO-PAN & 4.0 & 5.9 & 36 \\
\hline & LANL TiO, & 4.0 & 4.4 & 5.4 \\
\hline & Crech MnO-PAN & 3.0 & 4.3 & 4.8 \\
\hline & SNL/CST69 & 2.8 & 3.8 & 14 \\
\hline & Czech NaY-PAN & 2.3 & 4.1 & 4.8 \\
\hline & SNL/CSTIII & 2.1 & 1.9 & 1.7 \\
\hline & SNL/CST68 & 1.7 & 2.4 & 6.6 \\
\hline & SNL/CST84 & 1.4 & 1.7 & 8.8 \\
\hline & Sr-Specinl resin & 1.3 & 1.6 & 2.11 \\
\hline & SNL/CST48 & 1.3 & 1.7 & $0.1)$ \\
\hline & TRU-Specin resin & 1.3 & 1.9 & 2.5 \\
\hline & SNL/CST 35 & 1.1 & 1.9 & 1).6 \\
\hline & Duolite ${ }^{\mathrm{N}} \mathrm{CS}-100$ resin & 1.0 & 2.3 & 3.5 \\
\hline
\end{tabular}




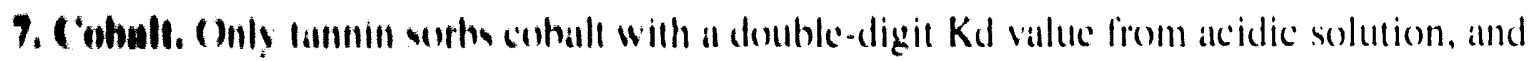

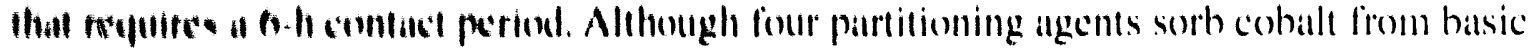

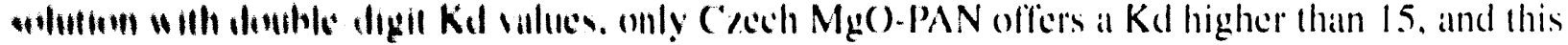

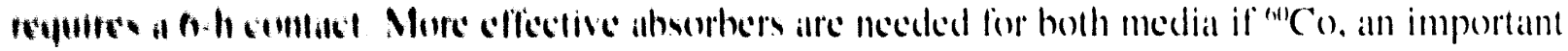

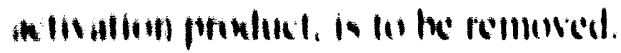

Tuble III.A.7. Cohbull Dissribution Ditli

\begin{tabular}{|c|c|c|c|c|}
\hline Madlum & Absorber & 3)-min Kd & $2 \cdot h \mathrm{Kd}$ & 6-h Kd \\
\hline \multirow[t]{8}{*}{ Achl: Dinulied Sludge } & Diphonix" resin & 1.4 & 1.7 & 2.1 \\
\hline & Cach MgO.PAN & 1.3 & 1.4 & 1.4 \\
\hline & Dunlite ${ }^{111} \mathrm{C} .467$ resin & 1.3 & 0.4 & 0.8 \\
\hline & C\%ch M.315.PAN & 1.3 & 1.0 & 1.1 \\
\hline & L.ANL $\mathrm{Zr},\left(\mathrm{PO}_{4}\right)_{4}$ & 1.3 & 1.3 & 1.1 \\
\hline & Tunnin & 1.3 & 3.5 & 13 \\
\hline & Amberlyst ${ }^{\prime N} \mathrm{XN}-1010$ resin & 1.3 & 0.9 & 1.2 \\
\hline & Ionsiv'M TIE:96 & 1.2 & 0.7 & 0.8 \\
\hline \multirow[t]{11}{*}{ Nkaline Sunpernate } & Dunlite ${ }^{1 N} \mathrm{C}-467 \mathrm{resin}$ & 14 & 13 & 12 \\
\hline & SRS resorcinol resin & 12 & 15 & 7.7 \\
\hline & Crech MgO-PAN & 9.4 & 15 & 33 \\
\hline & Cach NiFC-PAN & 8.9 & 10 & 11 \\
\hline & Crech NaTiO-PAN & 6.2 & 7.9 & 9.5 \\
\hline & SNL/CST68 & 5.3 & 5.4 & 2.3 \\
\hline & SNL/CST48 & 4.5 & 4.6 & 2.0 \\
\hline & Bunc charr & 4.4 & 5.5 & 6.9 \\
\hline & Amberlyst ${ }^{\prime N} \mathrm{XN}-1010$ resin & 4.0 & 3.5 & 3.4 \\
\hline & Amberlysit 1.5 resin & 3.6 & 3.5 & 3.1 \\
\hline & Crypt-DER resin & 2.1 & 2.1 & 2.1 \\
\hline
\end{tabular}


8. Iron. Iron is poorly sorbed by all absorbers from both acidic and basic media. This is fortunate because iron is present at high concentration in many waste solutions. Interference by iron in the sorption of other elements may therefore be much less than had been expected. When iron is present at high concentration, however, significant amounts may load even on the absorbers with low Kd values.

Table III.A.8. Iron Distribution Data

\begin{tabular}{|c|c|c|c|c|}
\hline Medium & Absorber & 30-min Kd & 2-h Kd & 6-h Kd \\
\hline \multirow{15}{*}{ Acid-Dissolved Sludge } & Czech NaY-PAN & 2.2 & 1.6 & 1.5 \\
\hline & $\mathrm{LIX}^{\mathrm{TM}}-84$ & 1.0 & 0.5 & 0.8 \\
\hline & Duolite ${ }^{T M}$ CS-100 resin & 0.8 & 0.8 & 1.4 \\
\hline & SNL/CST35 & 0.8 & 0.5 & 0.8 \\
\hline & Czech M315-PAN & 0.8 & 0.5 & 0.5 \\
\hline & Duolite ${ }^{\mathrm{rM}} \mathrm{C}-467$ & 0.8 & 0.2 & 0.4 \\
\hline & TRU-Spec ${ }^{\mathrm{TM}}$ resin & 0.8 & 0.8 & 0.8 \\
\hline & SNL/CST68 & 0.7 & 0.7 & 0.9 \\
\hline & $\mathrm{LIX}^{\mathrm{TM}}-54$ & 0.7 & 0.3 & 0.4 \\
\hline & SNL/CST48 & 0.7 & 1.0 & 1.0 \\
\hline & Ionsiv ${ }^{\text {TM }}$ TIE-96 & 0.7 & 0.4 & 0.8 \\
\hline & Czech MgO-PAN & 0.7 & 0.8 & 1.0 \\
\hline & Czech MnO-PAN & 0.7 & 1.2 & 1.0 \\
\hline & CMPO-DIPB & 0.6 & 0.6 & 0.5 \\
\hline & Amberlyst ${ }^{\mathrm{TM}} 15$ resin & 0.5 & 1.2 & 0.8 \\
\hline \multirow[t]{14}{*}{ Alkaline Supernate } & Czech MnO-PAN & 3.1 & 4.5 & 5.8 \\
\hline & $\mathrm{LIX}^{\mathrm{TM}}-54$ & 2.8 & 3.4 & 4.7 \\
\hline & SNL/CST69 & 2.2 & 2.0 & 2.2 \\
\hline & SNL/HTO & 2.2 & 3.1 & 3.9 \\
\hline & Diphonix $^{\mathrm{TM}}$ resin & 2.1 & 2.7 & 3.1 \\
\hline & Duolite $^{\mathrm{TM}} \mathrm{CS}-100$ resin & 1.8 & 2.6 & 3.2 \\
\hline & Czech NiFC-PAN & 1.4 & 1.7 & 2.3 \\
\hline & Czech TiO-PAN & 1.4 & 3.8 & 5.2 \\
\hline & Amberlyst ${ }^{\mathrm{TM}} 15$ resin & 1.2 & 3.4 & 5.8 \\
\hline & TRU-Spec ${ }^{\mathrm{TM}}$ resin & 0.8 & 1.4 & 2.5 \\
\hline & Reillex ${ }^{\mathrm{TM}} \mathrm{HPQ}$ & 0.7 & 0.8 & 1.2 \\
\hline & SNL/CST 111 & 0.6 & 1.6 & 1.9 \\
\hline & SNL/CST84 & 0.6 & 1.2 & 1.4 \\
\hline & $\mathrm{Sr}-\mathrm{Spec}^{\mathrm{TM}}$ resin & 0.3 & 0.8 & 1.5 \\
\hline
\end{tabular}


9. Manganese. Manganese is moderately sorbed by Duolite ${ }^{\mathrm{TM}} \mathrm{C}-467 \mathrm{resin}$ and Czech NaTiO-PAN from basic solution, but by no absorbers from acidic solution. As in the case of iron, this indicates that the interference from manganese may be less than expected.

Table III.A.9. Manganese Distribution Data

\begin{tabular}{|c|c|c|c|c|}
\hline Medium & Absorber & 0 -min Kd & 2-h Kd & 6-h Kd \\
\hline \multirow[t]{7}{*}{ Acid-Dissolved Sludge } & Diphonix $^{\mathrm{TM}}$ resin & 1.8 & 3.2 & 3.8 \\
\hline & Duolite ${ }^{\mathrm{TM}} \mathrm{C}-467$ resin & 1.3 & 1.3 & 1.4 \\
\hline & Amberlyst ${ }^{\mathrm{TM}} \mathrm{XN}-1010$ resin & 1.1 & 0.5 & 0.4 \\
\hline & PK-01-244 resin & 1.0 & 0.3 & 0.3 \\
\hline & $\mathrm{LIX}^{\mathrm{TM}}-84$ & 0.9 & 0.3 & 0.5 \\
\hline & Crypt-DER resin & 0.8 & 0.7 & 0.4 \\
\hline & Czech NaTiO-PAN & 0.4 & 0.4 & 0.4 \\
\hline \multirow[t]{13}{*}{ Alkaline Supernate } & Duolite ${ }^{\mathrm{TM}} \mathrm{C}-467$ resin & 15 & 17 & 17 \\
\hline & Czech NaTiO-PAN & 6.0 & 7.3 & 7.6 \\
\hline & SRS resorcinol resin & 5.9 & 4.7 & 2.2 \\
\hline & Amberlyst ${ }^{\mathrm{TM}} 15$ resin & 5.2 & 4.7 & 4.5 \\
\hline & $\mathrm{LIX}^{\mathrm{TM}}-1010$ & 4.9 & 5.7 & 5.3 \\
\hline & Amberlyst ${ }^{\mathrm{TM}} \mathrm{XN}-1010$ resin & 4.3 & 4.9 & 5.0 \\
\hline & $\mathrm{LIX}^{\mathrm{TM}}-54$ & 2.4 & 2.1 & 2.6 \\
\hline & SNL/CST48 & 2.0 & 1.7 & 1.2 \\
\hline & SNL/CST68 & 1.9 & 1.9 & 1.6 \\
\hline & Bone char & 1.8 & 2.5 & 2.9 \\
\hline & Czech MnO-PAN & 1.8 & 1.9 & 2.2 \\
\hline & CMPO-DIPB & 1.6 & 1.6 & 2.1 \\
\hline & Tannin & 1.3 & 0.4 & 0.5 \\
\hline
\end{tabular}


10. Zinc. None of the absorbers removes significant amounts of zinc from acidic solution: however, many atsorbers offer moderate-to-high $\mathrm{Kd}$ values from basic solution.

Table III.A.10. Zinc Distribution Data

\begin{tabular}{|c|c|c|c|c|}
\hline Medium & Absorber & 30-min Kd & 2-h Kd & 6-h Kd \\
\hline \multirow[t]{8}{*}{ Acid-Dissolved Sludge } & JSK-2 resin & 2.8 & 1.9 & 1.1 \\
\hline & JSK-3 resin & 1.7 & 1.3 & 0.7 \\
\hline & JSK-1 resin & 1.6 & 1.0 & 0.9 \\
\hline & Diphonix ${ }^{\mathrm{TM}}$ resin & 1.2 & 2.1 & 2.1 \\
\hline & Duolite $^{\mathrm{TM}} \mathrm{C}-467$ resin & 0.9 & 0.7 & 0.8 \\
\hline & PK-01-244 resin & 0.8 & 0.4 & 0.4 \\
\hline & SNL/CST111 & 0.8 & 0.6 & 0.6 \\
\hline & Crypt-DER resin & 0.7 & 0.5 & 0.3 \\
\hline \multirow[t]{10}{*}{ Alkaline Supernate } & Czech NiFC-PAN & 61 & 94 & 90 \\
\hline & SNL/CST68 & 33 & 44 & 43 \\
\hline & Duolite ${ }^{\mathrm{rM}} \mathrm{C}-467$ resin & 24 & 46 & 89 \\
\hline & SNL/CST48 & 23 & 32 & 34 \\
\hline & Czech NaTiO-PAN & 21 & 44 & 71 \\
\hline & SNL/HTO & 18 & 18 & 19 \\
\hline & SRS resorcinol resin & 9.3 & 11 & 9.0 \\
\hline & Bone char & 8.3 & 14 & 18 \\
\hline & $\mathrm{LIX}^{\mathrm{TM}}-1010$ & 3.6 & 4.0 & 4.0 \\
\hline & Amberlyst ${ }^{\mathrm{TM}} \mathrm{XN}-1010$ resin & 3.2 & 3.4 & 3.6 \\
\hline
\end{tabular}


11. Zirconium. Many absorbers offer moderate-to-high sorption of zirconium from acidic solution. A particularly interesting material is Czech TiO-PAN composite, which is highly selective for zirconium among the 14 elements studied. Only Czech MgO-PAN offers a double-digit $\mathrm{Kd}$ value for zirconium from basic solution. However, because the 6-h value for MnO-PAN is significantly higher than the values obtained for shorter contact times, this high value is suspect. The erratic Kd values for SNL/CST69 are attributed to incomplete exclusion of submicron absorber particles from the assay solution, and for SNL/CST84 to dissolution of an amorphous phase that sorbs zirconium.

12. Uranium. Many absorbers offer useful Kd values for uranium from acidic solution. Note that all 60 absorbers were evaluated from acid-dissolved sludge that contained $3 \mathrm{~g}{ }^{234} \mathrm{Pu} / \mathrm{L}$. A few selected absorbers also were evaluated from a similar solution that contained ${ }^{238} \mathrm{Pu}$, but at a 50-fold lower concentration, to determine how the lower plutonium concentration affected actinide Kd values. From basic solution, Cyanex ${ }^{\mathrm{N}} 923$ outperforms other much-costlier partitioning agents.

Table III.A.1 1. Zirconium Distribution Data

\begin{tabular}{|c|c|c|c|c|}
\hline Medium & Absorber & 30-min Kd & 2-h Kd & 6-h Kd \\
\hline \multirow[t]{14}{*}{ Acid-Dissolved Sludge } & Czech NaY-PAN & 107 & 191 & 196 \\
\hline & SNL/CST69 & 91 & 14 & 116 \\
\hline & Duracil $^{\mathrm{TM}} 230$ & 75 & 220 & 205 \\
\hline & SNL/CST84 & 70 & 87 & 19 \\
\hline & Czech TiO-PAN & 51 & 323 & 899 \\
\hline & Ionsiv'M TIE-96 (mod.) & 35 & 88 & 115 \\
\hline & SNL/CST68 & 31 & 119 & 103 \\
\hline & Ionsivin TIE-96 & 28 & 64 & 85 \\
\hline & Czech M315-PAN & 27 & 67 & 96 \\
\hline & SNL/CST48 & 15 & 49 & 51 \\
\hline & SNL/CST 111 & 11 & 14 & 18 \\
\hline & Duolite ${ }^{\mathrm{M}} \mathrm{CS}-100$ resin & 9.2 & 16 & 22 \\
\hline & SNL/CST35 & 8.2 & 26 & 76 \\
\hline & Cyanex $^{\mathrm{PM}} 272$ & 8.0 & 32 & 199 \\
\hline \multirow[t]{9}{*}{ Alkaline Supernate } & Amberlyst ${ }^{\mathrm{M}} 15$ resin & 2.8 & 4.6 & 8.4 \\
\hline & Czech MgO-PAN & 1.7 & 2.9 & 28 \\
\hline & SNL/HTO & 1.7 & 2.7 & 6.4 \\
\hline & SNL/CST69 & 1.7 & 2.5 & 5.5 \\
\hline & Czech MnO-PAN & 1.7 & 2.2 & 2.3 \\
\hline & Sr-Spec ${ }^{\mathrm{TM}}$ resin & 1.6 & 1.8 & 2.1 \\
\hline & Crech TiO-PAN & 1.5 & 2.0 & 3.2 \\
\hline & TRU-Spec ${ }^{\mathrm{M}}$ resin & 1.4 & 1.9 & 2.6 \\
\hline & Duolite ${ }^{\mathrm{TM}} \mathrm{CS}-100$ resin & 1.1 & 2.1 & 3.1 \\
\hline
\end{tabular}


Table III.A.12. Uranium Distribution Data

\begin{tabular}{|c|c|c|c|c|}
\hline Medium & Absorber & 30-min Kd & 2-h Kd & 6-h Kd \\
\hline \multirow{14}{*}{$\begin{array}{l}\text { Acid-Dissolved Sludge } \\
(3 \mathrm{~g} \mathrm{Pu} / \mathrm{L})\end{array}$} & TRU-Spec ${ }^{\mathrm{TM}}$ resin & 25 & 26 & 27 \\
\hline & JSK-2 resin & 19 & 19 & 19 \\
\hline & JSK-3 resin & 18 & 18 & 17 \\
\hline & Reillex ${ }^{\mid \mathrm{N}}$ HPQ resin & 16 & 19 & 18 \\
\hline & JSK-I resin & 14 & 13 & 12 \\
\hline & Sybron ${ }^{T M}(E t)_{3} N$ resin & 13 & 22 & 22 \\
\hline & Ionac ${ }^{\mathrm{TM}}$ SR-3 resin & 12 & 21 & 37 \\
\hline & Purolite ${ }^{\mathrm{M}} \mathrm{A}-520-\mathrm{E}$ resin & 9.9 & 24 & 35 \\
\hline & Sr-Spec ${ }^{\mid \mathrm{N}}$ resin & 4.9 & 5.1 & 5.7 \\
\hline & Duolite ${ }^{\mathrm{MM}} \mathrm{C}-467$ resin & 3.8 & 5.0 & 6.0 \\
\hline & Amberlyst ${ }^{\mathrm{rM}} 1.5$ resin & 3.1 & 6.4 & 7.2 \\
\hline & DHDECMP-DIPB & 1.2 & 2.3 & 2.7 \\
\hline & Diphonix $^{\mathrm{TN}}$ resin & 0.4 & 1.5 & 2.8 \\
\hline & CMPO-DIPB & 0.4 & 0.8 & 1.4 \\
\hline \multirow[t]{6}{*}{ (60 mg Pu/L) } & Reillex ${ }^{I M}$ HPQ resin & 20 & 2.3 & 23 \\
\hline & JSK-2 resin & 20 & 17 & 17 \\
\hline & JSK-3 resin & 18 & 17 & 17 \\
\hline & JSK-1 resin & 13 & 12 & 11 \\
\hline & Purolite ${ }^{\mathrm{M}} \mathrm{A}-520-\mathrm{E}$ resin & 10 & 22 & 39 \\
\hline & Cyanex ${ }^{\mathrm{TN}} 923$ & 2.7 & 6.5 & 14 \\
\hline \multirow[t]{17}{*}{ Alkaline Supernate } & Cyanex ${ }^{T M} 923$ & 122 & 23.3 & 356 \\
\hline & Diphonix ${ }^{1 M}$ resin & 96 & 1.53 & 214 \\
\hline & TRU-Spec ${ }^{\mathrm{TN}}$ resin & 70 & 107 & 164 \\
\hline & Czech TiO-PAN & 67 & 115 & 185 \\
\hline & SNL/HTO & 52 & 69 & 90 \\
\hline & CMPO-DIPB & 49) & 18.3 & 291 \\
\hline & SNL/CST35 & 2.5 & 45 & .54 \\
\hline & Cyanex ${ }^{\mathrm{IN}} 272$ & 23 & 41 & 58 \\
\hline & SNL/CST68 & 2.3 & 38 & 4.5 \\
\hline & DHDECMP & 22 & 31 & .32 \\
\hline & Duolite ${ }^{\mathrm{N} 1} \mathrm{C}-467$ resin & 19 & 38 & 6.3 \\
\hline & Amberlyst ${ }^{\mathrm{TN}} 15$ resin & 15 & 30 & 58 \\
\hline & Duolite ${ }^{\mathrm{TM}} \mathrm{CS}-100$ resin & 12 & 19 & 25 \\
\hline & Reillex ${ }^{1 M}$ HPQ resin & 8.4 & 8.9 & 8.9 \\
\hline & SRS resorcinol resin & 7.5 & 1.3 & 22 \\
\hline & Bone char & 6.1 & 9.6 & 13 \\
\hline & $\mathrm{Sr}^{-}-\mathrm{Spec}^{\mathrm{IN}}$ resin & 4.8 & 4.9 & 5.2 \\
\hline
\end{tabular}


13. Plutonium. Aside from the outstanding performance of the experimental JSK anion exchange resins, and Sybron ${ }^{1 N}$ (Et), $N$ resin for a 6 -h contact period. Reillex ${ }^{\text {N }}$ IIPQ sorbs plutonium from acidic solution better than all other partitioning agents. The performance of JSK -3 resin, which greally exceeds that of Reillex ${ }^{1 N}$ HPQ, is particulaty impressive. The poor removal of plutonium from basic solution may not be a serious problem because the solubility of plutonium in this medium is expected to be quite low.

Table Ill.A.13. Plutonium Distribution Dalta

\begin{tabular}{|c|c|c|c|c|}
\hline Medium & Absorber & 3()-min Kd & 2-h Kd & 6-h Kd \\
\hline \multirow{13}{*}{$\begin{array}{l}\text { Acid-Dissolved Sludge } \\
\text { (3g } \mathrm{P} u / \mathrm{L})\end{array}$} & JSK-3 resin & 10.53 & 10.3 .5 & 1173 \\
\hline & JSK-2 resin & 142 & 278 & 422 \\
\hline & JSK-I resin & 8.3 & 14.3 & 196 \\
\hline & Reillex ${ }^{1 \mathrm{~N}}$ HPQ resin & 78 & 274 & 48.5 \\
\hline & TRU-Spec rM resin & 57 & 58 & 6() \\
\hline & Sybron ${ }^{|N|}(E t), N$ resin & 24 & 114 & 8.39 \\
\hline & Ionacin SR-3 resin & 21 & 64 & 2() 7 \\
\hline & Sr-Spec ${ }^{1 \mathrm{~N}}$ resin & 14 & 14 & 14 \\
\hline & Purolite ${ }^{\mathrm{M}} \mathrm{A}-520-\mathrm{E}$ resin & 1.3 & 51 & 415 \\
\hline & DHDECMP & 4.4 & 8.2 & 15 \\
\hline & Duolite ${ }^{\text {IM }}$ CS-100 resin & 4.0 & 6.7 & 10 \\
\hline & Cyanex ${ }^{|\mathrm{N}|} 923$ & 3.0 & 7.6 & 21 \\
\hline & CMPO-DIPB & 0.4 & 0.4 & 0.7 \\
\hline \multirow[t]{6}{*}{ (60 mg Pu/L) } & JSK-3 resin & 87() & 16.50 & 218.5 \\
\hline & JSK-2 resin & 329 & 3.37 & 261 \\
\hline & JSK-I resin & 178 & 249 & 2.54 \\
\hline & Reillex ${ }^{\mathrm{IN}} \mathrm{HPQ}$ resin & 1.32 & 184 & 205 \\
\hline & Purolite ${ }^{\mathrm{N}} \mathrm{A}-520-$ E resin & 4.5 & 186 & 260 \\
\hline & Cyanex ${ }^{\mathrm{IN}} 923$ & 21 & 100 & 190 \\
\hline \multirow[t]{10}{*}{ Alkaline Supernate } & Crech MnO-PAN & 2.8 & 3.3 & 3.8 \\
\hline & Duolite ${ }^{|N|}$ CS-100) resin & 2.7 & 3.4 & 4.4 \\
\hline & Amberlyst 1.5 resin & 2.3 & 4.9 & 8.5 \\
\hline & $\operatorname{LIX}^{1 \mathrm{M}_{-} .54}$ & 2.2 & 2.8 & 3,0 \\
\hline & Cyanex ${ }^{1 N 1} 923$ & 1.4 & 1.9 & 2.0 \\
\hline & TRU-SpeciN resin & 1.2 & 2.0 & 2.8 \\
\hline & Diphonix ${ }^{1 M}$ resin & 1.0 & 1.2 & $1 .()$ \\
\hline & CMPO-DIPB & 0.8 & 0.5 & 1.0 \\
\hline & Reillex ${ }^{I N}$ HPQ resin & 0.7 & 0.9 & (). 7 \\
\hline & DHDECMP-DIPB & 0.7 & (). 6 & $(0.4$ \\
\hline
\end{tabular}


14. Americium. Americium is sorbed by the ISK experimental anion exchange resins and by Reillex ${ }^{T M}$ HPQ resin from acidic solution. but al lower levels than we would like. From basic

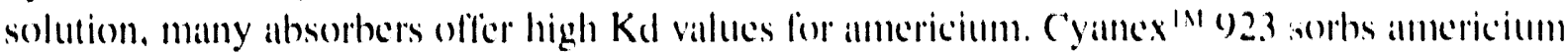
better than any other partitioning agent studied, including specialty products costing orders of magnitude more.

\section{B. Individual Absorbers}

Tables III.B. I.a. Uhrough III.B.7.g. present distribution coeflicients for eidch of the It elements onto each of the 60 ) absorbers, from simulated acid-dissolved sladge and alkaline supernate solutions of Hanford PFP Tank 102-SY. These tables may be used to predied the sclecetivity of specific absorbers being considered.

Table III.A.14. Americium Distribution Dalla

\begin{tabular}{|c|c|c|c|c|}
\hline Medium & Absorber & 3(1)-min Kd & 2-h Kd & $6.11 \mathrm{Kc}$ \\
\hline \multirow{6}{*}{$\begin{array}{l}\text { Acid-Dissolved Sludge } \\
(3 \mathrm{~g} \mathrm{Pu} / \mathrm{L})\end{array}$} & JSK-2 resin & 8.4 & 7.9 & 7.6 \\
\hline & JSK-I resin & 7.4 & 0.5 & 5.9 \\
\hline & JSK-3 resin & 6.2 & 5.3 & 5.0 \\
\hline & Reillex ${ }^{1 N}$ HPQ resin & 3.9 & 2.4 & 2.4 \\
\hline & TRU-Specin resin & $(0 . .3$ & $<() .1$ & $<() .1$ \\
\hline & Diphonix ${ }^{\mid \mathrm{N}}$ resin & $<(), 1$ & 0.3 & 0.4 \\
\hline \multirow[t]{4}{*}{$((6) \mathrm{mg} P \mathrm{Pu} / \mathrm{L})}$. & JSK-2 resin & 9.4 & 7.7 & 7.2 \\
\hline & JSK-I resin & 8.3 & 7.0 & 0.0 \\
\hline & JSK-.3 resin & 7.9 & $6 . .3$ & 0.0 \\
\hline & Reillex ${ }^{\text {IN }}$ HPQ resin & 4.6 & 3.6 & 3.8 \\
\hline \multirow[t]{15}{*}{ Alkuline Supermale } & (yannex ${ }^{1 N 1}$ ()2.3 & 5.38 & 7.51 & $8(1) 2$ \\
\hline & TRII-Specin resin & .195 & .548 & 671 \\
\hline & SNL/HTO & 229 & 204 & 168 \\
\hline & ( MPO-DIPB & $41)$ & 3.5 .5 & 472 \\
\hline & $1.1 X^{1 N 1} . .54$ & .34 & .32 & $3(1)$ \\
\hline & Amberlyst'N 1.5 resin & 3.3 & 68 & $1) .3$ \\
\hline & (rech MnO-PAN & .32 & $3(1)$ & 26 \\
\hline & Diphonix 1 resin & 28 & 2.5 & 22 \\
\hline & SNI./CSTIII & 2.5 & .52 & 107 \\
\hline & DHIDI:CMP & 25 & .34 & 3.5 \\
\hline & C'MCh $A M P-P A N$ & 24 & .5() & 86 \\
\hline & $1.1 x^{1 N}-26$ & 2.3 & 3.3 & $.3(1)$ \\
\hline & Bonc char & 16 & .35 & 02 \\
\hline & Amberlyst ${ }^{1 N 1} X N-1010$ resill & in 10 & 27 & $h()$ \\
\hline & $1.1 X^{|N|}-1(1) \mid 0$ & 10 & 27 & 6.1 \\
\hline
\end{tabular}


1. Commercially Available Absorbers. Seventecen commercially available absorbers were included in our study.

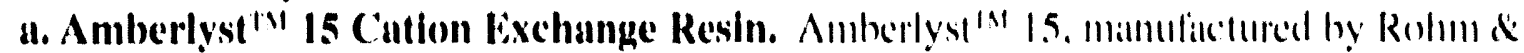

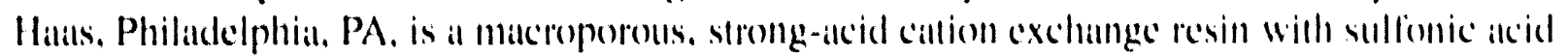
functional groups. Air-drying the as-received resin before use resuled in a weight loss of $4.5 .4 \%$.

Table III.B. I.a. shows that surprisingly few elements soth on his cillion exchange resin from

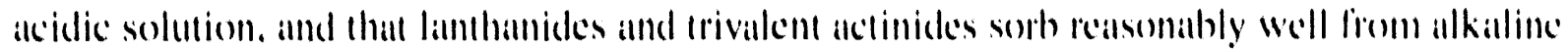
solution. Amberlyst in 15 is outperformed by only one other absorber for remosing stromtium from hasic solution.

Table III.B.1.a. Distribution of I I lements onto Amberlyst 1.5 ('illon Fixchange Resin from Tiwo Simulaun Solutions for Hantord HI.W Tank IO)2-SY

\begin{tabular}{|c|c|c|c|c|}
\hline Medium & Absorber & 3(1)-min Kd & 2-h Kd & (-) $/ \mathrm{Kd}$ \\
\hline \multirow[t]{14}{*}{ Acid-Dissolved Sludge } & $\mathrm{Co}$ & 2.0 & 1.7 & 1.8 \\
\hline & $C s$ & 1.5 & 1.3 & 1.6 \\
\hline & $\mathrm{Sr}$ & 1.2 & (1).9) & 1.2 \\
\hline & $\mathrm{Tc}$ & $1 .(1)$ & $(1) .7$ & 1.2 \\
\hline & $Y$ & 2.3 & 1.7 & 2.1 \\
\hline & $C^{\prime} r$ & $1 .(1)$ & 0.7 & $(0.9$ \\
\hline & (i) & $(1.9$ & ().6 & (1).6 \\
\hline & $\mathrm{Fc}$ & 0.5 & 1.2 & $(1.8$ \\
\hline & $M n$ & $(0.3$ & 0.6 & 0.4 \\
\hline & 7.11 & $<() .1$ & $<() .1$ & $<(1) .1$ \\
\hline & $\%$ & 1.2 & 1.3 & 1.3 \\
\hline & 11 & 3.1 & 0.4 & 7.2 \\
\hline & $P u$ & 1.1 & 1.9 & 1.8 \\
\hline & AIII & (1).7 & 0.7 & 11.6 \\
\hline \multirow[t]{14}{*}{ Alkilline Supermalle } & $c$ & 42 & 77 & 1.32 \\
\hline & ( ) & 1.7 & $1) 1$ & (1).(1) \\
\hline & $\mathrm{Sr}$ & 12 & 1.3 & 1.1 \\
\hline & $\mathrm{Ti}$ & $<() .1$ & $<1) .1$ & $<() .1$ \\
\hline & Y & 27 & 4.5 & $n$ \\
\hline & $C^{\prime} r$ & 4.2 & 7.9 & 1.4 \\
\hline & (') & 3.6 & 3.5 & 3.1 \\
\hline & $\mathrm{ICD}$ & 1.2 & 3.4 & 5.8 \\
\hline & $M \|$ & 5.2 & 4.7 & 4.5 \\
\hline & 7.11 & 2.4 & $2.1)$ & 1.8 \\
\hline & $7 x$ & 2.8 & 4.6 & 8.4 \\
\hline & 11 & 1.5 & .311 & .58 \\
\hline & $P_{u}$ & 2.3 & 4.9 & X.. \\
\hline & AIII & .33 & $6 x$ & (1).3 \\
\hline
\end{tabular}


b. Amberlyst 'M XN-1010 Cation lixchange Resin. Amberlyst'Ml XN-1010, a macroporous. strong-acid cation exchange resin with sulfonic acid functional groups, is mantalctured by Rohm \& Haus. Philadelphia, PA. Amberlyst XN-10)10 is stated by the mamulacturer (o) have a particu-

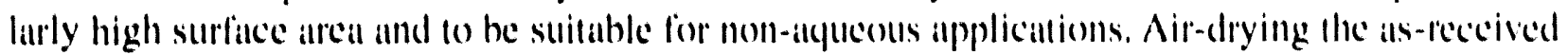
resin before use resulled in a weight loss of $4.4 \%$.

Table III.B.1.h. shows that the hehavior of Amberlyst ${ }^{\text {IN }}$ XN-1010 is similat (o that of Amberlyst ${ }^{1 N 1} 1.5$, although the Ko values generally are lower.

Tuble III.B.1.b. Distribution of 14 Elements onto Amberlyst XN-10)10 C'ulion Lixchange Resin from Two Simulant Solutions for Hanford HI.W Tank 102-SY

\begin{tabular}{|c|c|c|c|c|}
\hline Medlum & Alssorber & 3()$\cdot \mathrm{m} / \mathrm{n}$ Kd & 2-h Kd & 6-h Kd \\
\hline \multirow[t]{14}{*}{ Acid-Dissolved Sludge } & $\mathrm{Ce}$ & 1.3 & 1.4 & 1.5 \\
\hline & $\mathrm{Cs}$ & 2.4 & 2.2 & 2.5 \\
\hline & $\mathrm{Sr}$ & 0.9 & 0.9 & 1.1 \\
\hline & $\mathrm{Tc}$ & 1.2 & 1.1 & 1.3 \\
\hline & $Y$ & 1.4 & 1.4 & 1.5 \\
\hline & $\mathrm{Cr}$ & $(0.9$ & ().6 & 1.3 \\
\hline & $\mathrm{Co}$ & 1.3 & 0.9 & 1.2 \\
\hline & $\mathrm{Fe}$ & $(0.3$ & 1.1 & $(0.8$ \\
\hline & $\mathrm{Mn}$ & 1.1 & 0.5 & 0.4 \\
\hline & $Z n$ & $(0 . .3$ & $<() .1$ & $<() .1$ \\
\hline & $\mathrm{Zr}$ & 1.2 & 1.8 & 3.3 \\
\hline & U & 1.2 & 2.4 & 4.6 \\
\hline & $\mathrm{Pll}$ & $<() .1$ & (1).7 & 1.3 \\
\hline & AlII & 0.2 & (1.4 & 0.2 \\
\hline \multirow[t]{14}{*}{ Alkuline Supernule } & $\mathrm{Cc}$ & 1.3 & .38 & 114 \\
\hline & $\mathrm{Cs}$ & 1.3 & 1.5 & 15 \\
\hline & $\mathrm{Sr}$ & 5.9 & 7.8 & 8.3 \\
\hline & $\mathrm{Tc}$ & $1.3^{3}$ & 1.0 & $1.1)$ \\
\hline & $Y$ & 11 & 26 & 64 \\
\hline & $\mathrm{Cr}$ & $(1) .5$ & $(1) .8$ & 1.3 \\
\hline & Co & $4.1)$ & 3.5 & 3.4 \\
\hline & Fo & $<() .1$ & $<() .1$ & $<() .1$ \\
\hline & $\mathrm{Mn}$ & 4.3 & 4.9 & $5 .()$ \\
\hline & $\mathrm{Zn}$ & 3.2 & 3.4 & 3.6 \\
\hline & $\mathrm{Zr}$ & $<(), 1$ & $<() .1$ & $<() .1$ \\
\hline & 11 & $4.1)$ & 6.7 & 1.5 \\
\hline & $\mathrm{Pu}$ & 0.3 & 0.1 & 0.4 \\
\hline & Am & 10 & 27 & 66 \\
\hline
\end{tabular}


c. Duolite' C.467 Cutlon Lxchunge Resin. Duolite'N (-467. al chelating ciation cxchange resin composed of a polystyrene/divinylbenzene copolymer with aminophosphonic acid functional groups, is manufiactured by Rohm \& Hats. Philaddphia, PA. Air-drying he as-received resin before use resulled in a weight loss of $34.1 \%$

Table III.B.1.e. shows moderalle sorption of lechenetium and tirconium from alcidic solution. From basic solution, tedinedium, zince, and uranium sorb reasonably well, whereas cobalt, matnganese, annericium, and lanthanides sorb moderaltely.

Tuble III.13.1.c. Distribution of I t Elements onto Duolite" ('-467 Ciltion Exchange Resin from Two Simulant Solutions for Hanford HI.W Tank 102-SY

\begin{tabular}{|c|c|c|c|c|}
\hline Medium & Absorber & $3(1)-m i n ~ K d$ & 2-h Kd & 6-h Kd \\
\hline \multirow[t]{14}{*}{ Acid-Dissulved Sludge } & $\mathrm{Cc}$ & $2 . .4$ & 1.5 & 2.2 \\
\hline & $\mathrm{Cs}$ & (0.9) & (0.2 & 0.7 \\
\hline & $\mathrm{Sr}$ & 1.4 & 0.6 & $(0.9$ \\
\hline & $T c$ & 12 & 12 & 1.3 \\
\hline & $Y$ & 1.8 & 0.9 & 1.6 \\
\hline & $\mathrm{Cr}$ & 1.1 & (0.4 & 1.0 \\
\hline & $\mathrm{Co}$ & 1.3 & (1).4 & $(0.8$ \\
\hline & $\mathrm{Fe}$ & $(0.8$ & ().2 & 0.4 \\
\hline & $M n$ & $1.3^{3}$ & 1.3 & 1.4 \\
\hline & $\mathrm{Zn}$ & $(0,9$ & (1).7 & $(0.8$ \\
\hline & $\mathrm{Zr}$ & 5.4 & 7.7 & 1.3 \\
\hline & (1) & 3.8 & S.() & 6.0 \\
\hline & $P_{u}$ & 2.4 & 3.2 & 3.9 \\
\hline & AlII & $(1) .8$ & 0.7 & 0.7 \\
\hline \multirow[t]{14}{*}{ Alkuline Supernule } & $c$ & 6.3 & 4.2 & 3.6 \\
\hline & $\mathrm{Cs}$ & $<(), 1$ & $<(), 1$ & (i.) \\
\hline & $\mathrm{Sr}$ & 1.4 & 0.7 & $(0.5$ \\
\hline & $T \%$ & 4() & 42 & 44 \\
\hline & $Y$ & 7.2 & 5.6 & 4.5 \\
\hline & $\mathrm{Cr}$ & 0.5 & $(1) .7$ & 1.1 \\
\hline & (i) & 14 & 1.3 & 12 \\
\hline & lic & $<(1) .1$ & (1).6 & $(0.8$ \\
\hline & $M_{n}$ & 1.5 & 17 & 17 \\
\hline & 7.11 & 24 & 46 & 89 \\
\hline & $7 . r$ & $<(), 1$ & (1). & (1).3 \\
\hline & 11 & 10 & 38 & 6.3 \\
\hline & $P_{u}$ & 0.7 & 1.0 & 1.3 \\
\hline & AIII & $(0.9)$ & 0.2 & 5.4 \\
\hline
\end{tabular}


d. Diphonix" Cation Exchange Resin. Diphonix IM, a polyfinctional resin thall contains diphosphonic acid, sulfonic acid, and carboxylic acid functional gavops, was obtained from EIChroM Industries, Inc.. Darien, IL. Air-drying the as-received resin before use resulted in an unusually large weight loss of $72 \%$.

Diphonix ${ }^{\text {IN }}$ resin sorbs none of the 14 elements : ignificantly from acidic solution, whereas uranium, lanthanides, and americium sorb from basic solution.

Tuble III.B.1.d. Distribution of 14 Elements onto Diphonix ${ }^{1 M}$ Catton Exchange Resin from 'Two Simulant Solutions for Hanford HLW Tank 102-SY

\begin{tabular}{|c|c|c|c|c|}
\hline Medium & Absorber & 30-min Kd & 2-h Kd & 6-h Kd \\
\hline \multirow{14}{*}{ Acid-Dissolved Sludge } & $\mathrm{Ce}$ & 1.3 & 1.1 & 1.2 \\
\hline & $\mathrm{Cs}$ & 1.7 & 1.4 & 1.3 \\
\hline & $\mathrm{Sr}$ & $2 .()$ & 1.7 & 1.3 \\
\hline & $T_{c}$ & 2.0 & 1.6 & 1.8 \\
\hline & $Y$ & 1.1 & 1.1 & 1.0 \\
\hline & $\mathrm{Cr}$ & 1.1 & 0.6 & 0.8 \\
\hline & $C_{0}$ & 1.4 & 1.7 & 2.1 \\
\hline & $F_{c}$ & 0.2 & 0.6 & 0.3 \\
\hline & $\mathrm{Mn}$ & 1.8 & 3.2 & 3.8 \\
\hline & $Z_{n}$ & 1.2 & 2.1 & 2.1 \\
\hline & $\mathrm{Zr}$ & 1.0 & 0.6 & 1.0 \\
\hline & U & 0.4 & 1.5 & 2.8 \\
\hline & $P_{l l}$ & $<() .1$ & $<() .1$ & $<0.1$ \\
\hline & Am & $<() .1$ & 0.3 & 0.4 \\
\hline \multirow[t]{14}{*}{ Alkuline Supernitle } & $\mathrm{Co}$ & 22 & 2.3 & 22 \\
\hline & $\mathrm{Cs}$ & 1.5 & 1.0 & 1.1 \\
\hline & $\mathrm{Sr}$ & 0.6 & 0.2 & 0.2 \\
\hline & $\mathrm{Tc}$ & 1.0 & 1.0 & 1.4 \\
\hline & $\mathrm{Y}$ & 28 & 31 & 31 \\
\hline & $\mathrm{Cr}$ & 0.9 & 1.7 & 3.1 \\
\hline & $\mathrm{Co}_{0}$ & 1.1 & 1.2 & 0.9 \\
\hline & $\mathrm{Fc}$ & 2.1 & 2.7 & 3.1 \\
\hline & $\mathrm{Mn}$ & 0.6 & 0.7 & 0.6 \\
\hline & $Z n$ & ().4 & $<() .1$ & $<() .1$ \\
\hline & $\mathrm{Zr}$ & $<() .1$ & $<() .1$ & 0.3 \\
\hline & $U$ & 96 & 1.5 .3 & 214 \\
\hline & $P_{1 I}$ & 1.0 & 1.2 & 1.0 \\
\hline & Am & 28 & 25 & 22 \\
\hline
\end{tabular}


e. Ionsiv' TN TIE-96 Absorber. IonsiviN TIE-96 is a titanium-loaded zeolite manufactured by UOP Molecular Sieves Division, Moorestown, NJ. This absorber wals used as received.

Ionsiv TM TIE-96 sorbs zirconium well and cesium moderately from acidic solution. From basic solution, cesium is sorbed well and uranium is sorbed moderately.

Table III.B.1.e. Distribution of 14 Elements onto Ionsiv ${ }^{\text {MI }}$ TIE-96 Absorber from Two Simulant Solutions for Hanford HLW Tank 102-SY

\begin{tabular}{|c|c|c|c|c|}
\hline Medium & Absorber & 30-min Kd & 2-h Kd & 6-h Kd \\
\hline \multirow[t]{14}{*}{ Acid-Dissolved Sludge } & $\mathrm{Ce}$ & 1.8 & 1.1 & 1.5 \\
\hline & Cs & 7.6 & 7.9 & 8.3 \\
\hline & $\mathrm{Sr}$ & 1.9 & 1.0 & 1.4 \\
\hline & $\mathrm{Tc}$ & 1.7 & 1.6 & 1.5 \\
\hline & $Y$ & 1.5 & 0.9 & 1.2 \\
\hline & $\mathrm{Cr}$ & 1.4 & 0.8 & 1.1 \\
\hline & $\mathrm{Co}$ & 1.2 & 0.7 & 0.8 \\
\hline & $\mathrm{Fc}$ & 0.7 & 0.4 & 0.8 \\
\hline & $\mathrm{Mn}$ & 0.1 & 0.1 & 0.1 \\
\hline & $\mathrm{Zn}$ & 0.4 & 0.2 & 0.3 \\
\hline & $\mathrm{Zr}$ & 28 & 64 & 85 \\
\hline & $\mathrm{U}$ & $<0.1$ & 0.4 & 0.1 \\
\hline & $\mathrm{Pu}$ & 1.5 & 2.5 & 4.4 \\
\hline & $A m$ & 0.2 & $<0.1$ & 0.3 \\
\hline \multirow[t]{14}{*}{ Alkaline Supernate } & $\mathrm{Ce}$ & 0.1 & 1.2 & 1.6 \\
\hline & Cs & 58 & 97 & 122 \\
\hline & $\mathrm{Sr}$ & 0.5 & 0.7 & 0.4 \\
\hline & $\mathrm{Tc}$ & $<0.1$ & $<0.1$ & $<0.1$ \\
\hline & $\mathrm{Y}$ & 0.9 & 1.3 & 1.1 \\
\hline & $\mathrm{Cr}$ & $<0.1$ & 0.9 & 0.9 \\
\hline & Co & 0.2 & 0.6 & 0.4 \\
\hline & $\mathrm{Fe}$ & 0.5 & 0.4 & 0.5 \\
\hline & $\mathrm{Mn}$ & 0.4 & 0.3 & 0.3 \\
\hline & $\mathrm{Zn}$ & 0.6 & 0.2 & 0.4 \\
\hline & $\mathrm{Zr}$ & $<0.1$ & 0.6 & 0.5 \\
\hline & $\mathrm{U}$ & 5.4 & 7.6 & 12 \\
\hline & $\mathrm{Pu}$ & $<0.1$ & 0.3 & 0.3 \\
\hline & $A m$ & 1.5 & 1.9 & 2.2 \\
\hline
\end{tabular}




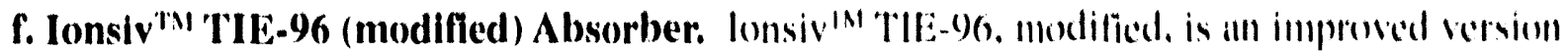
of the Lonsiv TIE-96 titanium-londed zeolite manufactured by (I)P Molecular Siever Division. Moorestown, NJ. This absorber was used as received.

The modified form of Ionsivin TIE-9) provides a generally improved version al alle sorption pattern of unmodified lonsiv IM TIE-96, with the Kds of lanthanides. Irimium, and antericium from basic solution approximately doubled.

Table III.B.1.f. Distribution of I4 Elements onto lonsiv IN TIE-96 (Modilied) Absorther from Two Simulant Solutions for Hanford HLW Tank 1()2-SY

\begin{tabular}{|c|c|c|c|c|}
\hline Medium & Absorber & 30)-min Kd & 2-h Kd & 6.h Kd \\
\hline \multirow[t]{14}{*}{ Acid-Dissolved Sludge } & $\mathrm{Ce}$ & 0.9 & 1.1 & 1.3 \\
\hline & $\mathrm{Cs}$ & 7.7 & (9.1) & 9.4 \\
\hline & $\mathrm{Sr}$ & 1.4 & 1.4 & 1.4 \\
\hline & $\mathrm{Tc}$ & 1.0 & 1.0 & $1 . .5$ \\
\hline & $Y$ & 1.2 & 1.0 & $1.1)$ \\
\hline & $\mathrm{Cr}$ & 0.4 & 0.9 & 1.1 \\
\hline & $\mathrm{Co}$ & 0.7 & 0.8 & 0.6 \\
\hline & $\mathrm{Fe}$ & 0.2 & 0.7 & 0.6 \\
\hline & $\mathrm{Mn}$ & 0.3 & 0.1 & 0.1 \\
\hline & $\mathrm{Zn}$ & 0.3 & (). 2 & (). 1 \\
\hline & $\mathrm{Zr}$ & 3.5 & 88 & 11.5 \\
\hline & U & $<0.1$ & 0.2 & 0.3 \\
\hline & $\mathrm{Pu}$ & 1.8 & 3.0 & 4.9 \\
\hline & $A m$ & 0.3 & 0.1 & ()... \\
\hline \multirow[t]{14}{*}{ Alkaline Supernate } & $\mathrm{Ce}$ & 2.3 & 3.0 & 2.9 \\
\hline & C s & 6.3 & 110 & 1.34 \\
\hline & $\mathrm{Sr}$ & 0.9 & 1.0 & (1). \\
\hline & $\mathrm{Tc}$ & $<() .1$ & $<() .1$ & $<() .1$ \\
\hline & $\mathrm{Y}$ & 2.2 & 2.2 & 1.9 \\
\hline & $\mathrm{Cr}$ & 1.0 & 1.6 & $1 . .3$ \\
\hline & $\mathrm{Co}$ & 1.0 & ().8 & $(1) .8$ \\
\hline & $\mathrm{Fe}$ & 0.5 & (). 6 & (1). 8 \\
\hline & $\mathrm{Mn}$ & (). .3 & 0.4 & 0.5 \\
\hline & $\mathrm{Zn}$ & 0.8 & (). .3 & $(0.5$ \\
\hline & $\mathrm{Zr}$ & 0.6 & 0.8 & (1).6 \\
\hline & $\mathrm{U}$ & 7.3 & 14 & 20 \\
\hline & $\mathrm{Pu}$ & $<() .1$ & 0.4 & (1). 2 \\
\hline & Am & 4.6 & 5.6 & 5.7 \\
\hline
\end{tabular}


n. Duolite" CS-100 Resin. Duolite ${ }^{\text {IN }}$ CS-100) is a phenolic resin manufactured by Rohun \& Has, Philadelphia, PA. Air-drying the as-received resin before use resulted in a weight loss of $55 \%$.

Dublite'" $(S-1(0)$ resin sorbs zirconium, plutonium, and technetium (but not cesium) from atidic solution. Only uranium is sorbed to a useful extent from basic solution.

Table III.B.1.g. Distribution of 14 Elements onto Duolite ${ }^{\mathrm{TM}}$ CS-100 Cation Exchange Revin from Two Simliant Solutions for Hanford HLW Tank 102-SY

\begin{tabular}{|c|c|c|c|c|}
\hline Medium & Absorber & 30-min Kd & 2-h Kd & 6-h Kd \\
\hline \multirow[t]{14}{*}{ Acid-Dissolved Sludge } & $\mathrm{Ce}$ & 0.2 & 0.5 & 0.5 \\
\hline & $\mathrm{Cs}$ & 0.5 & 1.1 & 1.3 \\
\hline & $\mathrm{Sr}$ & $<0.1$ & $<0.1$ & $<0.1$ \\
\hline & $\mathrm{Tc}$ & 4.8 & 6.3 & 6.4 \\
\hline & $\mathrm{Y}$ & 0.2 & 0.3 & 0.2 \\
\hline & $\mathrm{Cr}$ & 0.1 & 0.1 & 0.2 \\
\hline & $\mathrm{Co}$ & 0.3 & 0.5 & 0.4 \\
\hline & $\mathrm{Fe}$ & 0.8 & 0.8 & 1.4 \\
\hline & $\mathrm{Mn}$ & 0.1 & 0.1 & 0.3 \\
\hline & $\mathrm{Zn}$ & $<0.1$ & $<0.1$ & 0.2 \\
\hline & $\mathrm{Zr}$ & 9.2 & 16 & 22 \\
\hline & $\mathrm{U}$ & 1.2 & 1.6 & 2.4 \\
\hline & $\mathrm{Pu}$ & 4.0 & 6.7 & 10 \\
\hline & $\mathrm{Am}$ & 0.1 & $<0.1$ & $<0.1$ \\
\hline \multirow[t]{14}{*}{ Alkuline Supernate } & $\mathrm{Ce}$ & 2.6 & 2.5 & 3.0 \\
\hline & $\mathrm{Cs}$ & 1.3 & 1.2 & 1.3 \\
\hline & $\mathrm{Sr}$ & $<0.1$ & $<0.1$ & $<0.1$ \\
\hline & $\mathrm{Tc}$ & 2.2 & 2.6 & 2.8 \\
\hline & $\mathrm{Y}$ & 2.4 & 2.4 & 2.4 \\
\hline & $\mathrm{Cr}$ & 1.0 & 2.3 & 3.5 \\
\hline & Co & 0.5 & 0.4 & 0.5 \\
\hline & $\mathrm{Fe}$ & 1.8 & 2.6 & 3.2 \\
\hline & $\mathrm{Mn}$ & $<0.1$ & $<0.1$ & $<0.1$ \\
\hline & $\mathrm{Zn}$ & 1.1 & 0.9 & 0.8 \\
\hline & $\mathrm{Zr}$ & $1 . i$ & 2.1 & 3.1 \\
\hline & $\mathrm{U}$ & 12 & 19 & 25 \\
\hline & $\mathrm{Pu}$ & 2.7 & 3.4 & 4.4 \\
\hline & $\mathrm{Am}$ & 3.1 & 3.0 & 2.9 \\
\hline
\end{tabular}


h. Tannin Absorber. Tannin absurber was obtained from Mitsubishi Nuclear Fuels Corporation, Japan. Air-drying the as-received tannin resulted in an unusually high weight loss of $82.2 \%$.

Tannin sorbs technetium and zirconium from acidic solution, although the sorption kinetics are slow. Only uranium is sorbed with a Kd higher than 5 from basic solution, and again the sorption kinetics appear slow.

Table III.B.1.h. Distribution of 14 Elements onto Tannin Absorber from Two Simulant Solutions for Hanford HLW Tank 102-SY

\begin{tabular}{|c|c|c|c|c|}
\hline Medium & Absorber & 30-min Kd & 2-h Kd & 6-h Kd \\
\hline \multirow[t]{14}{*}{ Acid-Dissolved Sludge } & $\mathrm{Ce}$ & 1.0 & 0.6 & 0.5 \\
\hline & $\mathrm{Cs}$ & 1.8 & 1.5 & 1.3 \\
\hline & $\mathrm{Sr}$ & 0.9 & 0.6 & 0.3 \\
\hline & $\mathrm{Tc}$ & 10 & 39 & 136 \\
\hline & $\mathrm{Y}$ & 1.2 & 1.2 & 0.7 \\
\hline & $\mathrm{Cr}$ & 0.6 & 0.6 & 0.3 \\
\hline & $\mathrm{Co}$ & 1.3 & 3.4 & 13 \\
\hline & $\mathrm{Fe}$ & 0.3 & 0.2 & 0.7 \\
\hline & $\mathrm{Mn}$ & 0.2 & 0.3 & 0.2 \\
\hline & $\mathrm{Zn}$ & 0.4 & 0.3 & 0.2 \\
\hline & $\mathrm{Zr}$ & 4.3 & 12 & 33 \\
\hline & $\mathrm{U}$ & $<0.1$ & 0.6 & 1.0 \\
\hline & $\mathrm{Pu}$ & $<0.1$ & 0.6 & 2.5 \\
\hline & $\mathrm{Am}$ & 0.2 & 0.1 & 0.2 \\
\hline \multirow[t]{14}{*}{ Alkaline Supernate } & $\mathrm{Ce}$ & 1.9 & 3.7 & 4.1 \\
\hline & $\mathrm{Cs}$ & 2.4 & 2.2 & 2.4 \\
\hline & $\mathrm{Sr}$ & 1.0 & 0.9 & 0.7 \\
\hline & $\mathrm{Tc}$ & 2.1 & 2.7 & 2.7 \\
\hline & $Y$ & 1.8 & 3.3 & 4.1 \\
\hline & $\mathrm{Cr}$ & 0.1 & 0.3 & 0.6 \\
\hline & Co & 1.6 & 1.6 & 1.0 \\
\hline & $\mathrm{Fe}$ & $<0.1$ & $<0.1$ & 0.2 \\
\hline & $\mathrm{Mn}$ & 1.3 & 0.4 & 0.5 \\
\hline & $\mathrm{Zn}$ & 0.6 & 0.2 & 0.2 \\
\hline & $\mathrm{Zr}$ & $<0.1$ & $<0.1$ & $<0.1$ \\
\hline & $\mathrm{U}$ & 2.2 & 4.2 & 8.8 \\
\hline & $\mathrm{Pu}$ & 0.4 & 0.2 & 0.4 \\
\hline & Am & 1.1 & 2.0 & 3.1 \\
\hline
\end{tabular}


i. Sr-Spec ${ }^{\mathrm{TM}}$ Resin. Sr-Spec ${ }^{\mathrm{TM}}$ resin, a crown-ether-based material, was obtained from EIChroM Industries, Inc., Darien, IL. This resin was used as received.

The low $\mathrm{Kd}$ of 3 for strontium from acid is nearly matched by that for technetium, and is exceeded by Kds for uranium and plutonium. Technetium, strontium, and uranium sorb from basic solution. Some solution discoloration, observed in basic solution, indicated that $\mathrm{Sr}-\mathrm{Spec}{ }^{\mathrm{IN}}$ might be somewhat unstable in this medium.

Table III.B.1.i. Distribution of 14 Elements onto Sr-Spec ${ }^{\mathrm{TM}}$ Resin from Two Simulant Solutions for Hanford HLW Tank 102-SY

\begin{tabular}{|c|c|c|c|c|}
\hline Medium & Absorber & 30-min Kd & 2-h Kd & 6-h Kd \\
\hline \multirow[t]{14}{*}{ Acid-Dissolved Sludge } & $\mathrm{Ce}$ & 0.3 & 0.1 & 0.3 \\
\hline & $\mathrm{Cs}$ & 0.3 & 0.1 & 0.1 \\
\hline & $\mathrm{Sr}$ & 3.0 & 3.1 & 2.9 \\
\hline & $\mathrm{Tc}$ & 2.8 & 2.7 & 2.8 \\
\hline & $\mathrm{Y}$ & 0.6 & 0.4 & 0.5 \\
\hline & $\mathrm{Cr}$ & $<0.1$ & 0.1 & 0.2 \\
\hline & Co & 0.4 & 0.1 & 0.2 \\
\hline & $\mathrm{Fe}$ & $<0.1$ & 0.2 & 0.2 \\
\hline & $\mathrm{Mn}$ & 0.2 & 0.1 & 0.3 \\
\hline & $\mathrm{Zn}$ & 0.1 & 0.2 & 0.2 \\
\hline & $\mathrm{Zr}$ & 1.1 & 1.5 & 1.6 \\
\hline & $\mathrm{U}$ & 4.9 & 5.1 & 5.7 \\
\hline & $\mathrm{Pu}$ & 14 & 14 & 14 \\
\hline & $\mathrm{Am}$ & 0.1 & 0.2 & 0.1 \\
\hline \multirow[t]{14}{*}{ Alkaline Supernate } & $\mathrm{Ce}$ & 1.0 & 0.8 & 0.8 \\
\hline & Cs & 0.9 & 0.9 & 0.9 \\
\hline & $\mathrm{Sr}$ & 18 & 18 & 17 \\
\hline & $\mathrm{Tc}$ & 45 & 42 & 39 \\
\hline & $\mathrm{Y}$ & $<0.1$ & $<0.1$ & $<0.1$ \\
\hline & $\mathrm{Cr}$ & 1.3 & 1.6 & 2.0 \\
\hline & $\mathrm{Co}$ & 1.1 & 0.8 & 0.8 \\
\hline & $\mathrm{Fe}$ & 0.3 & 0.8 & 1.5 \\
\hline & $\mathrm{Mn}$ & 0.1 & 0.3 & 0.4 \\
\hline & $\mathrm{Zn}$ & $<0.1$ & $<0.1$ & $<0.1$ \\
\hline & $\mathrm{Zr}$ & 1.6 & 1.8 & 2.1 \\
\hline & $U$ & 4.8 & 4.9 & 5.2 \\
\hline & $\mathrm{Pu}$ & 0.4 & 0.7 & 1.0 \\
\hline & $\mathrm{Am}$ & 0.1 & 0.5 & 0.3 \\
\hline
\end{tabular}


j. TRU-Spec ${ }^{\text {TMI }}$ Resin. TRU-Spec ${ }^{\mathrm{IM}}$ resin, a mixture of $13 \%$ CMPO (octylphenyl-N, N-diisobutylcarbamoylmethylphosphine oxide) and 27\% TBP (tributyl phosphate) sorbed on polyacrylic ester beads, was obtained from EIChroM Industries, Inc., Darien, IL. This absorber was used as received.

Plutonium and uranium sorb reasonably well from acid, while technetium and zirconium sorb weakly. Lanthanides, americium, uranium, and technetium sorb well from basic solution.

Table III.B.1.j. Distribution of 14 Elements onto TRU-Spec ${ }^{\mathrm{IN}}$ Resin from Two Simulant Solutions for Hanford HLW Tank 102-SY

\begin{tabular}{|c|c|c|c|c|}
\hline Mr dium & Absorber & 30-min Kd & 2-h Kd & 6-h Kd \\
\hline \multirow[t]{14}{*}{ Acid-Dissolved Sludge } & $\mathrm{Ce}$ & 0.1 & 0.2 & 0.4 \\
\hline & $\mathrm{Cs}$ & 0.2 & 0.3 & 0.3 \\
\hline & $\mathrm{Sr}$ & 0.2 & 0.2 & 0.3 \\
\hline & $\mathrm{Tc}$ & 4.1 & 4.0 & 4.1 \\
\hline & $Y$ & 0.1 & 0.3 & 0.4 \\
\hline & $\mathrm{Cr}$ & 0.6 & 0.6 & 0.8 \\
\hline & Co & $<0.1$ & $<0.1$ & $<0.1$ \\
\hline & $\mathrm{Fe}$ & 0.8 & 0.8 & 0.8 \\
\hline & $\mathrm{Mn}$ & $<0.1$ & 0.1 & 0.4 \\
\hline & $\mathrm{Zn}$ & 0.6 & 0.5 & 0.6 \\
\hline & $\mathrm{Zr}$ & 3.8 & 4.3 & 4.9 \\
\hline & $\mathrm{U}$ & 25 & 26 & 27 \\
\hline & $\mathrm{Pu}$ & 57 & 58 & 60 \\
\hline & $\mathrm{Am}$ & 0.3 & 0.1 & 0.1 \\
\hline \multirow[t]{14}{*}{ Alkaline Supernate } & $\mathrm{Ce}$ & 548 & 594 & 644 \\
\hline & $\mathrm{Cs}$ & 1.5 & 1.8 & 2.0 \\
\hline & $\mathrm{Sr}$ & 0.5 & 0.2 & 0.1 \\
\hline & $\mathrm{Tc}$ & 90 & 78 & 74 \\
\hline & $\mathrm{Y}$ & 98 & 94 & 92 \\
\hline & $\mathrm{Cr}$ & 1.3 & 1.9 & 2.5 \\
\hline & Co & $<0.1$ & $<0.1$ & $<0.1$ \\
\hline & $\mathrm{Fe}$ & 0.8 & 1.4 & 2.5 \\
\hline & $\mathrm{Mn}$ & 0.3 & 0.3 & 0.3 \\
\hline & $\mathrm{Zn}$ & $<0.1$ & $<0.1$ & $<0.1$ \\
\hline & $\mathrm{Zr}$ & 1.4 & 1.9 & 2.6 \\
\hline & $\mathrm{U}$ & 70 & 107 & 164 \\
\hline & $\mathrm{Pu}$ & 1.2 & 2.0 & 2.8 \\
\hline & Am & 495 & 598 & 671 \\
\hline
\end{tabular}


k. Durasil ${ }^{\text {Mn }} 190$ Resin. Durasil ${ }^{\text {tM }} 190$ resin, obtained from Durallek Corporation. Beltsville, MD, was used as received. The alr-dried weight, per waller-wet ml, was (0.969) $\mathrm{g}$.

None of the 14 elements sorbs significantly from acidic solution, but cesium sorbs from basic solution.

Table III.B.1.k. Distribution of 14 Elements onto Durasil ${ }^{\text {IM }} 19()$ Resin from Two Simulant Solutions for Hanford HLW Tank 102-SY

\begin{tabular}{|c|c|c|c|c|}
\hline Medium & Absorber & 30-min Kd & 2-h Kd & 6-h Kd \\
\hline \multirow[t]{14}{*}{ Acid-Dissolved Sludge } & $\mathrm{Ce}$ & 0.4 & 0.5 & 0.7 \\
\hline & $\mathrm{Cs}$ & 1.2 & 1.7 & 2.5 \\
\hline & $\mathrm{Sr}$ & 0.1 & $<0.1$ & 0.3 \\
\hline & $\mathrm{Tc}$ & 0.5 & 0.5 & 0.7 \\
\hline & $\mathrm{Y}$ & $<0.1$ & 0.3 & 0.1 \\
\hline & $\mathrm{Cr}$ & 0.1 & $<0.1$ & 0.1 \\
\hline & $\mathrm{Co}$ & 0.3 & 0.3 & 0.3 \\
\hline & $\mathrm{Fe}$ & 0.3 & 0.6 & 0.7 \\
\hline & $\mathrm{Mn}$ & 0.3 & 0.4 & 0.2 \\
\hline & $\mathrm{Zn}$ & 0.4 & 0.6 & 0.5 \\
\hline & $\mathrm{Zr}$ & 0.7 & 1.3 & 1.7 \\
\hline & U & 0.4 & 0.1 & 0.2 \\
\hline & $\mathrm{Pu}$ & 0.2 & 0.2 & 0.2 \\
\hline & $\mathrm{Am}$ & $<0.1$ & 0.3 & 0.4 \\
\hline \multirow[t]{14}{*}{ Alkaline Supernate } & $\mathrm{Ce}$ & 0.3 & 0.1 & 0.4 \\
\hline & Cs & 9.4 & 21 & 34 \\
\hline & $\mathrm{Sr}$ & 0.1 & $<0.1$ & $<0.1$ \\
\hline & $\mathrm{Tc}$ & 0.4 & 0.3 & 0.4 \\
\hline & $\mathrm{Y}$ & $<0.1$ & 0.2 & 0.3 \\
\hline & $\mathrm{Cr}$ & 0.1 & 0.1 & 0.3 \\
\hline & Co & 0.4 & 0.3 & (0). 3 \\
\hline & $\mathrm{Fe}$ & 0.3 & 0.8 & 0.8 \\
\hline & $\mathrm{Mn}$ & $<0.1$ & $<0.1$ & $<0.1$ \\
\hline & $\mathrm{Zn}$ & 0.4 & 0.6 & 0.6 \\
\hline & $\mathrm{Zr}$ & 0.3 & 0.2 & 0.3 \\
\hline & U & 0.2 & 0.7 & 0.8 \\
\hline & $\mathrm{Pu}$ & 0.8 & 1.0 & 0.9 \\
\hline & $\mathrm{Am}$ & 0.1 & 0.6 & 0.6 \\
\hline
\end{tabular}




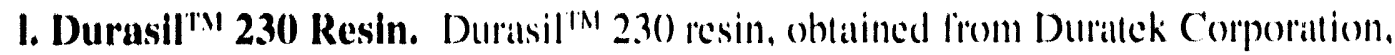
Beltsville, MD, was used as received. The air-dried weight, per walter-wet ml, wals $0.749 \mathrm{~g}$.

Zirconium and cesium sorb from acidic solution. Cesium sorbs selectively from basic solution.

Table 1II.B.1.I. Distribution of 14 Elements onto Durasil' ${ }^{\text {M }} 2.3($ Resin from Two Simulant Solutions for Hanford HLW Tank 102-SY

\begin{tabular}{|c|c|c|c|c|}
\hline Medium & Absorber & 30-min Kd & 2-h Kd & 6-h Kd \\
\hline \multirow{14}{*}{ Acid-Dissolved Sludge } & $\mathrm{Ce}$ & 0.3 & 0.5 & 0.5 \\
\hline & Cs & 11 & 14 & 1.3 \\
\hline & $\mathrm{Sr}$ & 0.5 & 0.1 & 0.2 \\
\hline & $\mathrm{Tc}$ & 0.5 & 0.7 & 0.6 \\
\hline & Y & 0.2 & 0.2 & 0.2 \\
\hline & $\mathrm{Cr}$ & $<(0.1$ & $<(0.1$ & $<0.1$ \\
\hline & Co & 0.4 & 0.3 & 0.4 \\
\hline & $\mathrm{Fe}$ & $<0.1$ & 0.1 & 0.2 \\
\hline & $\mathrm{Mn}$ & $<0.1$ & $<0.1$ & $<0,1$ \\
\hline & $\mathrm{Zn}$ & 0.1 & 0.1 & 0.1 \\
\hline & $\mathrm{Zr}$ & 75 & 220 & 20.5 \\
\hline & U & 0.4 & 0.3 & 0.3 \\
\hline & $\mathrm{Pu}$ & 1.2 & 2.8 & 3.4 \\
\hline & $A m$ & 0.3 & 0.1 & 0.2 \\
\hline \multirow[t]{14}{*}{ Alkaline Supernate } & $\mathrm{Ce}$ & $<0.1$ & 0.1 & $<0.1$ \\
\hline & $\mathrm{Cs}$ & 78 & 1.35 & 148 \\
\hline & $\mathrm{Sr}$ & 0.3 & 0.3 & 0.2 \\
\hline & $\mathrm{Tc}$ & 0.4 & 0.5 & 0.6 \\
\hline & $Y$ & 0.3 & 0.3 & 0.3 \\
\hline & $\mathrm{Cr}$ & 0.4 & 0.6 & 0.7 \\
\hline & Co & 0.1 & $<0.1$ & 0.3 \\
\hline & $\mathrm{Fe}$ & $<0.1$ & 0.4 & 0.9 \\
\hline & $\mathrm{Mn}$ & 0.3 & 0.3 & 0.3 \\
\hline & $\mathrm{Zn}$ & 0.4 & 0.4 & 0.7 \\
\hline & $\mathrm{Zr}$ & 0.4 & 0.7 & 0.9 \\
\hline & U & 0.7 & 0.5 & 0.9 \\
\hline & $\mathrm{Pu}$ & $<0.1$ & 0.3 & 0.7 \\
\hline & $\mathrm{Am}$ & 0.1 & $<0.1$ & 0.1 \\
\hline
\end{tabular}




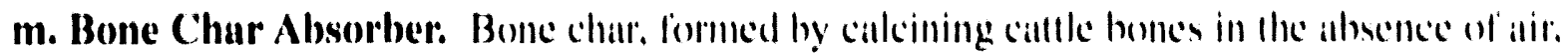
was ohtained from Stauffer Chemical Company, Westport. C"T. Bonc chatr is predominamlly callcium phosphate.

None of the It elements sorbs at useful levels from acidic solution, hull lanthamides, almericium, lechnetium, cobalt, and zinc sorb from basic solution.

n. Reillex ${ }^{\text {WI }}$ HPQ Anion lixchange Resin. Reillex IN IIPQ, a strong-hase polyvinylpyridinc anion exchange resin, was obtained from Reilly Industries. Inc.. Indianapolis, IN. This resin was converted from the as-received chloride form to the nitrate form and air-dried helofe use. "The alldried weight. per walter-wet ml, was $0.352 \mathrm{~g}$.

Table III.B.1.m. Distribution of I t Flements onto Bone Char Abourber from Tiwo Simulant Solutions for Hanford HLW Tank I()2-SY

\begin{tabular}{|c|c|c|c|c|}
\hline Medium & Alssorber & $3(1)-\min K d$ & $2-h \mathrm{Kll}$ & 6-: Kal \\
\hline \multirow[t]{14}{*}{ Acid-Dissolved Sludge } & $\mathrm{Ce}$ & 1.1 & 1.4 & 1.2 \\
\hline & $\mathrm{Cs}$ & 1.1 & 1.0 & 0.9 \\
\hline & $\mathrm{Sr}$ & 0.7 & 0.6 & $(0.8$ \\
\hline & $\mathrm{Tc}$ & $4 .()$ & $4.1)$ & 3.9 \\
\hline & Y & 0.6 & 0.4 & 0.5 \\
\hline & $\mathrm{Cr}$ & 0.7 & 0.5 & 0.7 \\
\hline & $\mathrm{Co}$ & 0.7 & 1.0 & 1.0 \\
\hline & $\mathrm{Fo}$ & 0.4 & 0.2 & (0).3 \\
\hline & $\mathrm{Mn}$ & 0.3 & 0.1 & ()..3 \\
\hline & $7 n$ & 0.4 & 0.2 & ()..3 \\
\hline & 7, & 2.0 & 2.5 & 2.6 \\
\hline & $\mathrm{U}$ & 1.5 & 0.9 & 1.1 \\
\hline & $P_{u}$ & 2.0 & 1.6 & 1.6 \\
\hline & Am & 0.6 & 0.4 & ()..3 \\
\hline \multirow[t]{14}{*}{ Alkuline Supernate } & $\mathrm{Ce}$ & 19 & 3.3 & 5.3 \\
\hline & $\mathrm{Cs}$ & 0.2 & 0.1 & 0.2 \\
\hline & $\mathrm{Sr}$ & 1.6 & 2.0 & 1.9 \\
\hline & $\mathrm{Tc}$ & 12 & 14 & 1.5 \\
\hline & $\mathrm{Y}$ & 11 & 18 & 26 \\
\hline & $\mathrm{Cr}$ & 0.2 & 0.4 & 0.7 \\
\hline & $\mathrm{Co}$ & 4.4 & 5.5 & 0.9 \\
\hline & $\mathrm{Fe}$ & 0.1 & (). .5 & 0.7 \\
\hline & $\mathrm{Mn}$ & 1.8 & 2.5 & 2.9 \\
\hline & $7 . n$ & 8.3 & 14 & 18 \\
\hline & $7 r$ & $<(0.1$ & 0.2 & 0.3 \\
\hline & $\mathrm{U}$ & 6.1 & 9.0 & 1.3 \\
\hline & $\mathrm{Pu}$ & 0.1 & 0.2 & 1.1 \\
\hline & Am & 16 & 3.5 & 62 \\
\hline
\end{tabular}




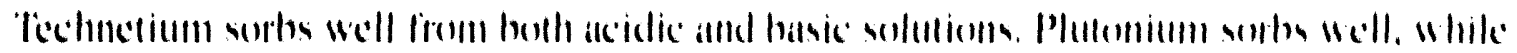

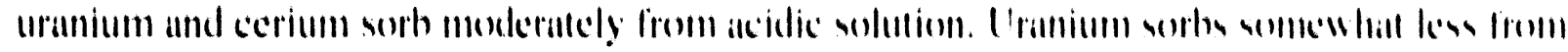
basice solution.

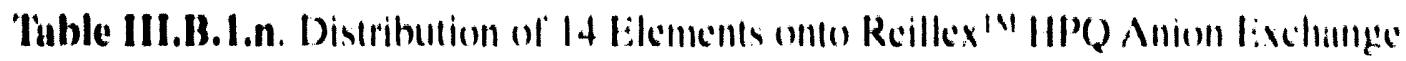
Resin from 'fwo Simulant Solutions for Hanford HI.W Tank 1012-SY

\begin{tabular}{|c|c|c|c|c|}
\hline Medium & Absorber & $30 \cdot \mathrm{m} / \mathrm{n}$ Kid & $2 \cdot h \mathrm{Kl}$ & $0 . h \mathrm{Kd}$ \\
\hline \multirow[t]{17}{*}{ Acid-Dissolved Sludge } & $C$ & 14 & 1.1 & 1.1 \\
\hline & $C$ & 0.8 & $(0.3$ & (1).6 \\
\hline & $\mathrm{Sr}$ & 1.1 & $(1) .6$ & 11.7 \\
\hline & $T c$ & 70 & $\varphi(1)$ & ฯ3 \\
\hline & $Y$ & 1.2 & 0.5 & (1).8 \\
\hline & $\mathrm{Cr}$ & $(1) .8$ & (1).2 & (1).6 \\
\hline & $\mathrm{Co}$ & 0.9 & (1).2. & (1).2 \\
\hline & $F$ & 0.5 & 0.5 & 0.5 \\
\hline & $M n$ & $<() .1$ & $<() .1$ & $<() .1$ \\
\hline & $Z n$ & $(0.3$ & 0.2 & 0.2 \\
\hline & $Z r$ & 4.3 & 4.2 & 4.2 \\
\hline & 11 & 17 & 19 & 18 \\
\hline & 11.1 & 20 & 23 & 2.3 \\
\hline & $\mathrm{Pu}$ & 78 & 274 & 48.5 \\
\hline & $P u^{\prime \prime}$ & 1.31 & 184 & $2(1) 5$ \\
\hline & Am & 3.9 & 2.4 & 2.4 \\
\hline & Am" & 4.6 & 3.6 & 3.8 \\
\hline \multirow[t]{14}{*}{ Alkaline Supernale } & $\mathrm{Ce}$ & $(0.4$ & (1).6 & 0.5 \\
\hline & $\mathrm{Cs}$ & 1.0 & $(1) .8$ & $(1) .8$ \\
\hline & $\mathrm{Sr}$ & $(0.1$ & ()..3 & (1). \\
\hline & $T c$ & 2.50 & 28() & 274 \\
\hline & $Y$ & 0.8 & 1.0 & (1).6 \\
\hline & $\mathrm{Cr}$ & $<() .1$ & $<(), 1$ & $<(1) .1$ \\
\hline & Co & $(0,9$ & 1.11 & (1).8 \\
\hline & $F$ & 0.7 & $(1) .8$ & 1.2 \\
\hline & $M n$ & $<() .1$ & $<(1), 1$ & $<(1) .1$ \\
\hline & $7 . n$ & 0.6 & 0.5 & (1).6 \\
\hline & $Z r$ & (). 2 & 0.4 & (1).4 \\
\hline & (I) & 8.4 & 8.9 & 8.9 \\
\hline & $\mathrm{Pl}$ & 0.7 & (1). & 0.7 \\
\hline & Am & 1.0 & (0.9) & 0.8 \\
\hline
\end{tabular}




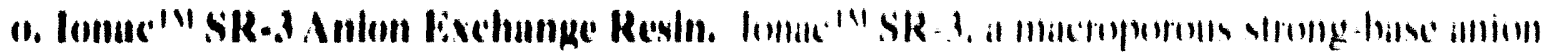

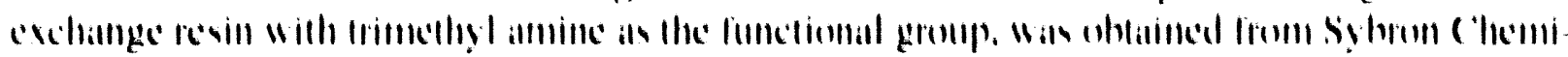

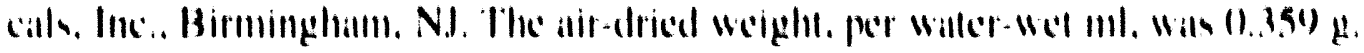

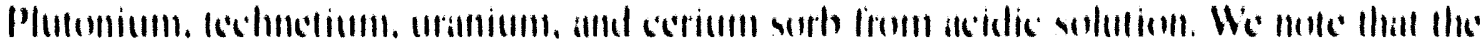

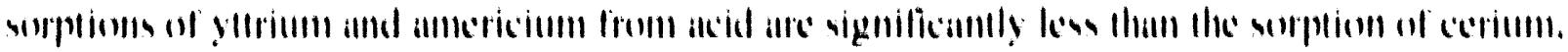

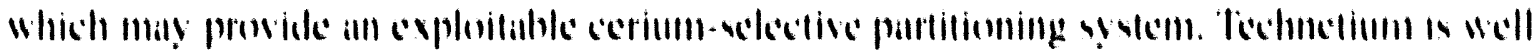

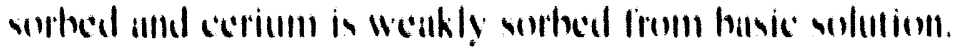

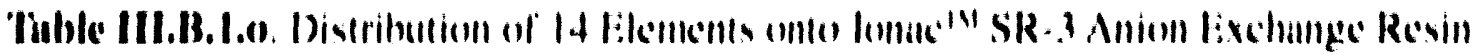

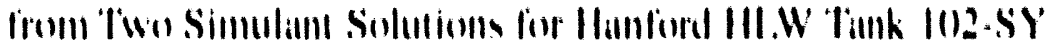

\begin{tabular}{|c|c|c|c|c|}
\hline Medlum & Alsowrber & 311-min Kd & $2 \cdot h$ Kd & 6-h Kd \\
\hline \multirow[t]{14}{*}{ Acid-Dismolved Sludge } & $C$ & 0.0 & 1.3 & 21 \\
\hline & C & $<() .1$ & $<(1) .1$ & $<(1.1$ \\
\hline & $\mathrm{Sr}$ & 11.2 & 11.0 & $(1), 3$ \\
\hline & $\mathrm{Tc}$ & 32 & 44 & 4.1 \\
\hline & $Y$ & 1.5 & 1.7 & 1.4 \\
\hline & $\mathrm{Cr}$ & 0.5 & 11.9 & 0.7 \\
\hline & (1) & $<() .1$ & 0.3 & 0.1 \\
\hline & Fis & (1). .3 & 0.5 & 0.6 \\
\hline & $M n$ & $<(1) .1$ & $<(1.1$ & $<(1) .1$ \\
\hline & $Z n$ & $(1) .1$ & 0.2 & 0.5 \\
\hline & $\% r$ & 1.4 & 2.8 & 2.4 \\
\hline & 11 & 12 & 21 & .17 \\
\hline & $P_{11}$ & 21 & 04 & 207 \\
\hline & AIII & 2.6 & 3.7 & 4.3 \\
\hline \multirow[t]{14}{*}{ Alkuline Supermale } & $\mathrm{Co}$ & 3.5 & 3.5 & .3 .4 \\
\hline & cis & $(1.5$ & $(1.5$ & (1). 4 \\
\hline & $\mathrm{Sr}$ & 1.0 & 0.7 & $(1) .9$ \\
\hline & $T c$ & 121 & 12.3 & 12.5 \\
\hline & $Y$ & 0.7 & (1).6 & 0.7 \\
\hline & $\mathrm{Cr}$ & $<() .1$ & $<() .1$ & $<(1) .1$ \\
\hline & (') & $(0,8$ & $(1) .8$ & 0.7 \\
\hline & lic & $<(1) .1$ & $(1) .3$ & $(0.1$ \\
\hline & Mn & (1)..3 & $(1) .5$ & $(1) .3$ \\
\hline & $7 . n$ & (1)..3 & $(1) .4$ & (1). 4 \\
\hline & $\mathrm{Zr}$ & 0.5 & 0.2 & $\left(0.3^{3}\right.$ \\
\hline & U & 1.2 & 1.6 & 1.7 \\
\hline & $P u$ & 0.5 & 0.1 & (1).2 \\
\hline & Am & (1).5 & (1). 4 & $(1) .8$ \\
\hline
\end{tabular}




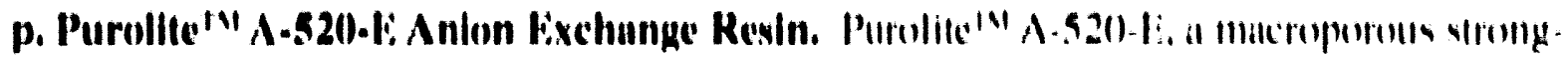

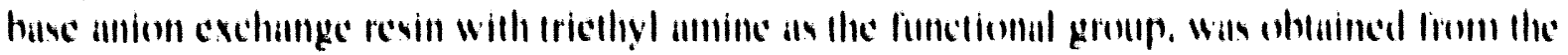

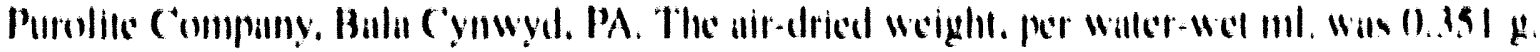

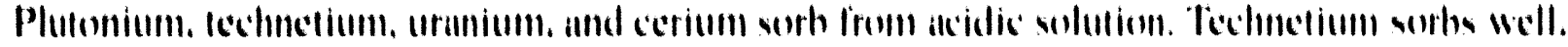
wherens cerium and urminim sorth slighlly from hasic solution.

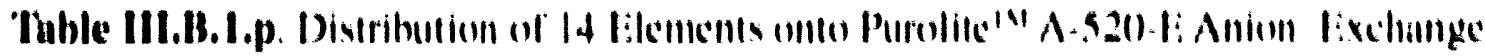

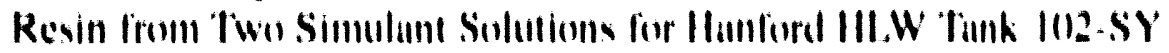

\begin{tabular}{|c|c|c|c|c|}
\hline Medium & Alsworber & X).min Kd & 2.h Kid & h.h Kel \\
\hline \multirow[t]{17}{*}{ Acid-Diswolved Sludge } & $\mathrm{Ce}$ & 7.8 & 14 & 21 \\
\hline & $\mathrm{Cs}$ & $<(1) .1$ & $<11.1$ & 0.1 \\
\hline & $\mathrm{Sr}$ & $<() .1$ & $(1) .1$ & $<(1) .1$ \\
\hline & $\mathrm{Tc}$ & .12 & 57 & 77 \\
\hline & $\mathbf{Y}$ & 1.4 & 1.1 & 1.1 \\
\hline & $\mathrm{Cr}$ & 0.5 & (1). 4 & 0.5 \\
\hline & (1) & 0.2 & 0.2 & $(1.3$ \\
\hline & $\mathrm{Fo}$ & 0.2 & 0.4 & 0.4 \\
\hline & Mn & $<() .1$ & $<() .1$ & $<() .1$ \\
\hline & Z.n & 0.3 & 0.3 & $(1) .3$ \\
\hline & $\mathrm{Zr}$ & 2.1 & 2.4 & 2.1 \\
\hline & 11 & $\mid(1) .1$ & 24 & .35 \\
\hline & $U^{1+}$ & 9.9 & 22 & 31) \\
\hline & $P u$ & 1.3 & $\$ 1$ & 41.5 \\
\hline & $P u^{\prime \prime}$ & 45 & 180 & $2(1)$ \\
\hline & AIII & 2.9 & 4.2 & 3.2 \\
\hline & $\Lambda n 1^{\prime \prime}$ & 2.7 & 4.2 & 3.7 \\
\hline \multirow[t]{14}{*}{ Alkuline Supermule } & $\mathrm{Ce}$ & 4.8 & 5.3 & 6.1 \\
\hline & Cs & 0.7 & 0.2 & 11.1 \\
\hline & $\mathbf{S r}$ & (1).6 & 0.4 & 11.5 \\
\hline & $\mathrm{Te}$ & 205 & .32() & $381)$ \\
\hline & $Y$ & 11.0 & 01.4 & 11.5 \\
\hline & C'r & $<() .1$ & $<(1) .1$ & $<(1) .1$ \\
\hline & Cil & (1.) & $(1.5$ & 11.6 \\
\hline & lis & 11.9 & 11.6 & 11.5 \\
\hline & $M n$ & $<(1) .1$ & 11.2 & 11.2 \\
\hline & 7.11 & 0.5 & 11.4 & (1).2 \\
\hline & $\%$ & 11.7 & (1). 3 & $(1) .1$ \\
\hline & 11 & 3.3 & 4.6 & 4.1 \\
\hline & $P \| I$ & $(1) .3$ & (1). 1 & 11.2 \\
\hline & AIII & (1).6 & 10.7 & (1).6 \\
\hline
\end{tabular}

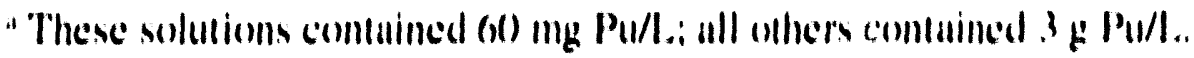




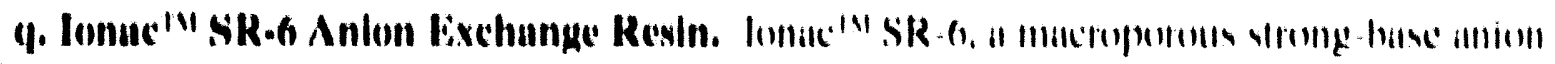

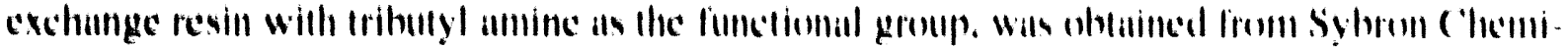

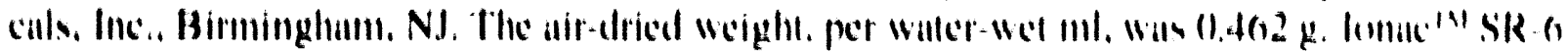

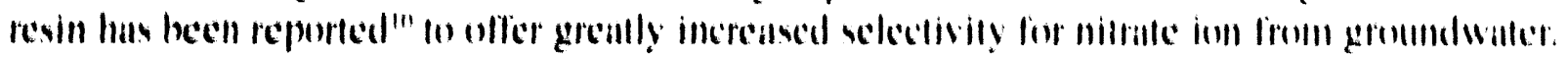

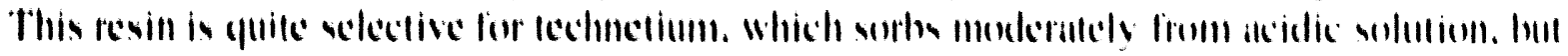
whos strongly from basic solution.

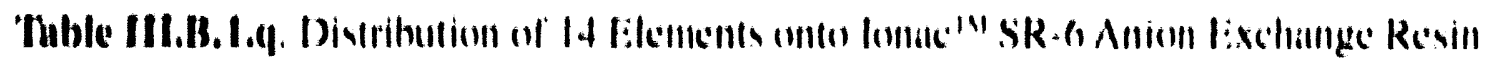
from two Simmlann Solutionn for Hannlond III.W Timk IO2.SY

\begin{tabular}{|c|c|c|c|c|}
\hline Medlum & Absorber & $3(1) \cdot m i n ~ K d l$ & $2 \cdot h \mathrm{Kd}$ & o.h Kd \\
\hline \multirow[t]{14}{*}{ Acidabiswolved Sludge } & $C$ & 0.6 & 0.7 & 0.7 \\
\hline & Cr & 0.3 & 0.3 & $(1) .4$ \\
\hline & $\mathrm{Sr}$ & 0.1 & 0.7 & 0.4 \\
\hline & $\mathrm{Tc}$ & 2.2 & 4.0 & 8.1 \\
\hline & $\mathbf{Y}$ & 0.1 & $(1) .4$ & $(1) .1$ \\
\hline & $\mathrm{Cr}$ & $(1) .2$ & 0.1 & 11.5 \\
\hline & (') & $<(1.1$ & $<(1) .1$ & $<() .1$ \\
\hline & 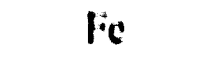 & 0.1 & 0.3 & $(1) .8$ \\
\hline & $M_{n}$ & $<(1) .1$ & $<() .1$ & $<(1.1$ \\
\hline & $Z 11$ & 0.2 & 11.2 & 0.3 \\
\hline & $7 r$ & $<(1) .1$ & 10.5 & 11.0 \\
\hline & 11 & 0.2 & $(1) .8$ & 0.7 \\
\hline & $P_{11}$ & 0.1 & 0.5 & $(1.5$ \\
\hline & AIII & $(1) .1$ & 01.3 & $(1) .3$ \\
\hline \multirow[t]{14}{*}{ Alkuline Supermule } & $\mathrm{Cu}$ & $<(1) .1$ & 11.2 & $(1.1$ \\
\hline & Cis & 0.3 & (1). 1 & 0.1 \\
\hline & $\mathrm{Sr}$ & $(0.1$ & 0.3 & 0.3 \\
\hline & $\mathrm{TH}$ & 1.51 & 3114 & 010 \\
\hline & $Y$ & 0.4 & 0.5 & 11.4 \\
\hline & C'r & 0.4 & (1).6 & $(1) .8$ \\
\hline & (i) & 0.2 & 0.4 & (1).6 \\
\hline & $\mathrm{Fo}$ & $(1.1$ & $<(1) .1$ & 0.2 \\
\hline & Mn & 0.5 & $(1) .2$ & 0.2 \\
\hline & 7.11 & (1).2 & 0.3 & 10.5 \\
\hline & 7 & 0.2 & 11.3 & $(1) .3$ \\
\hline & !! & 11.6 & 1.3 & 1.7 \\
\hline & $P_{11}$ & $(1) .7$ & 0.2 & 11.5 \\
\hline & AIII & $<() .1$ & $<(1) .1$ & $<1) .1$ \\
\hline
\end{tabular}


2. Lexperimentul Absorbers. Light experimental absorbers were included in our study.

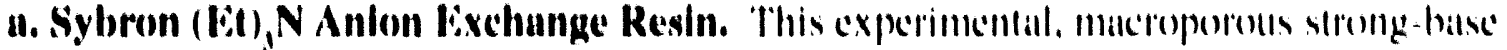
anion exchange resin with triethyl annine as the functional group, was obtained from Sybrom Chemicals, Birmingham. NJ. The air-dried weight, per waller-we mil, was (0.36.5 g. This resin was shown in a previous study" Io signilicanly increase lle sorption of Pu(IV) from dilute nitric acid.

The behavion of this experimental resin is similar to that of Purolite $\AA .52(1)$ f: resin (its comllmercial equivalent), except for the lower sorpeion of errium from alkaline solution.

Tuble III.R.2.n. Distribution of I E Elements onto Sybron (lit), N Anion Exchange Resin from Two Simulant Solutions for Hanford HLW Tank 102.SY

\begin{tabular}{|c|c|c|c|c|}
\hline Medlum & Absorber & $30-\mathrm{min} \mathrm{Kd}$ & 2-h Kd & 6.h Kd \\
\hline \multirow[t]{14}{*}{ Acid-Dissolved Sludge } & Ce & 7.4 & 9.6 & 7.5 \\
\hline & $\mathrm{Cs}$ & 0.2 & 0.1 & 0.2 \\
\hline & $\mathrm{Sr}$ & 0.1 & (). 2 & (). .3 \\
\hline & $T c$ & 31 & 51 & 5.3 \\
\hline & $Y$ & 0.6 & 0.6 & $(0 . .3$ \\
\hline & $\mathrm{Cr}$ & 0.3 & 0.1 & 0.4 \\
\hline & $\mathrm{Co}$ & $<0.1$ & $<() .1$ & $<(0.1$ \\
\hline & $\mathrm{Fc}$ & 0.1 & 0.1 & 0.4 \\
\hline & $M n$ & $<0.1$ & $<() .1$ & 0.3 \\
\hline & $\mathrm{Zn}$ & 0.3 & 0.2 & 0.5 \\
\hline & $\mathrm{Zr}$ & 2.4 & 2.6 & 2.0 \\
\hline & $U$ & 1.3 & 22 & 22 \\
\hline & $\mathrm{Pu}_{\mathrm{u}}$ & 24 & 114 & 8.39 \\
\hline & Am & 2.6 & 1.4 & 0.8 \\
\hline \multirow[t]{14}{*}{ Alkuline Supernule } & $\mathrm{Co}$ & 0.7 & 0.7 & 0.8 \\
\hline & $\mathrm{Cs}$ & $<0.1$ & $<() .1$ & $<() .1$ \\
\hline & $\mathrm{Sr}$ & 0.2 & 0.4 & 0.3 \\
\hline & $\mathrm{Tc}$ & 264 & $4(05$ & 44() \\
\hline & $Y$ & 0.2 & 0.5 & 0.4 \\
\hline & $\mathrm{Cr}$ & 0.8 & 0.9 & 0.8 \\
\hline & $\mathrm{Co}$ & 0.2 & 0.5 & 0.4 \\
\hline & $\mathrm{Fe}$ & 0.1 & 0.2 & ().2 \\
\hline & $\mathrm{Mn}$ & 0.3 & (). 3 & 0.4 \\
\hline & $\mathrm{Zn}$ & 0.5 & ().5 & 0.3 \\
\hline & $\mathrm{Zr}$ & 0.1 & 0.1 & 0.3 \\
\hline & U & 2.8 & 3.1 & 3.1 \\
\hline & $P l l$ & $<0.1$ & $<() .1$ & 0.2 \\
\hline & Am & 0.3 & 0.4 & (0.2 \\
\hline
\end{tabular}


b. Sybron ( $\mathrm{Pr})_{3} \mathbf{N}$ Anion Exchange Resin. This cxperimental, macroporous strong-hatse anion exchange resin with tripropyl amine as the functional group. Was obtained from Sybron Chemicals, Birmingham, N.J. The air-dried weight, per water-wel ml, was $0.4($ ) g.

Only echnetium and aranium are signiticantly sorbed from acidic solution, whereas cerium is weakly sorbed. Technelium is well sorbed, and uranium is weakly sorbed, from balsic solution.

Table III.B.2.b. Distribution of 14 Elements onto Sybron ( $\mathrm{Pr}$ ), $\mathrm{N}$ Anion Exchange Resin from Two Simulant Solutions for Hanford HLW Tank 1()2-SY

\begin{tabular}{|c|c|c|c|c|}
\hline Medium & Absorber & 30-min Kd & 2.h Kd & 6-h Kd \\
\hline \multirow[t]{14}{*}{ Acid-Dissolved Sludge } & $\mathrm{Ce}$ & 1.2 & 1.7 & 2.2 \\
\hline & Cs & 0.1 & 0.5 & 0.3 \\
\hline & $\mathrm{Sr}$ & $<0.1$ & 0.4 & 0.2 \\
\hline & $\mathrm{Tc}$ & 11 & 26 & 4.5 \\
\hline & $Y$ & 0.2 & 0.5 & 0.3 \\
\hline & $\mathrm{Cr}$ & $<0.1$ & 0.8 & 0.6 \\
\hline & Co & 0.3 & 0.1 & 0.1 \\
\hline & $\mathrm{Fe}$ & 0.1 & 0.3 & 0.3 \\
\hline & $\mathrm{Mn}$ & 0.1 & $<0.1$ & $<0.1$ \\
\hline & $\mathrm{Zn}$ & $<0.1$ & 0.4 & 0.2 \\
\hline & $\mathrm{Zr}$ & 0.5 & 1.0 & 1.8 \\
\hline & $\mathrm{U}$ & 1.9 & 3.5 & 7.1 \\
\hline & $\mathrm{Pu}$ & 0.7 & 1.7 & 3.2 \\
\hline & Am & 0.3 & 0.7 & 0.9 \\
\hline \multirow[t]{14}{*}{ Alkuline Supernalle } & $\mathrm{Ce}$ & 0.4 & 0.1 & 0.2 \\
\hline & $\mathrm{Cs}$ & $<0.1$ & $<0.1$ & $<() .1$ \\
\hline & $\mathrm{Sr}$ & 0.3 & 0.2 & 0.3 \\
\hline & $\mathrm{Tc}$ & 401 & 777 & 992 \\
\hline & $\mathrm{Y}$ & 0.4 & 0.5 & 0.4 \\
\hline & $\mathrm{Cr}$ & 0.6 & 0.6 & 0,6 \\
\hline & $\mathrm{Co}$ & 0.4 & 0.6 & 0.4 \\
\hline & $\mathrm{Fc}$ & 0.3 & 0.2 & 0.1 \\
\hline & $\mathrm{Mn}$ & $<0.1$ & 0.2 & 0.1 \\
\hline & $\mathrm{Zn}$ & 0.7 & 0.4 & 0.4 \\
\hline & $\mathrm{Zr}$ & 1.2 & 0.4 & 0.7 \\
\hline & U & 3.5 & 4.2 & 5.4 \\
\hline & $P_{u}$ & 0.5 & 0.4 & 0.3 \\
\hline & Amı & 0.3 & 0.1 & 0.1 \\
\hline
\end{tabular}


The three following JSK experimental resins are custom-symlhesized anton sexchungers whose composition cannot be disclosed until our patent position is sectlred. We cian stalls, however, lhat although these resins involve a new concept for anion exchange, llay can be produced hy moditying existing commercial resins. For this reason. production on a commercial sciale may be considerably less difficult than would be the cinse for an entirely new product.

c. JSK-1 Anion Exchange Resin. From acidic solution, plutonium is strongly sorbed, whereas cerium, lechnetium, and uranium sorb at lower, bul useful levels. Zirconium and yltrium sorption from acid are low. Only technetium sorbs well from basic solution: zinc, urimium, and americium sorb slightly from basic solution.

Table III.B.2.c. Distribution of 14 Elements onto JSK-I Experimental Anion Exchange Resin from Two Simulant Solutions for Hanford HLW Tank 102-SY

\begin{tabular}{|c|c|c|c|c|}
\hline Medium & Absorber & 30-min Kd & 2.h Kd & G.h Kd \\
\hline Acid-Dissolved Sludge & $\begin{array}{l}C e \\
C s \\
S r \\
T c \\
Y \\
C r \\
C 0 \\
F e \\
M n \\
Z n \\
Z r \\
U \\
U^{\prime} \\
P^{\prime} \\
P u^{\prime} \\
A m \\
A m^{\prime}\end{array}$ & $\begin{array}{c}28 \\
0.4 \\
1.2 \\
3.2 \\
1.7 \\
0.5 \\
<0.1 \\
0.1 \\
<0.1 \\
1.6 \\
4.7 \\
14 \\
1.3 \\
8.3 \\
178 \\
7.4 \\
8.3\end{array}$ & $\begin{array}{c}29 \\
0.1 \\
1.3 \\
32 \\
1.2 \\
0.4 \\
0.3 \\
0.1 \\
<0.1 \\
1.1 \\
4.4 \\
1.3 \\
12 \\
14.3 \\
249 \\
0.5 \\
7.0\end{array}$ & $\begin{array}{c}27 \\
0.2 \\
1.1 \\
.10 \\
1.1 \\
0.4 \\
0.1 \\
0.2 \\
<0.1 \\
0.9 \\
2.8 \\
12 \\
11 \\
196 \\
2.54 \\
5.9 \\
6.6\end{array}$ \\
\hline Alkaline Supernate & $\begin{array}{l}\mathrm{Ce} \\
\mathrm{Cs} \\
\mathrm{Sr} \\
\mathrm{Tc} \\
\mathrm{Y} \\
\mathrm{Cr} \\
\mathrm{Co} \\
\mathrm{Fe} \\
\mathrm{Mn} \\
\mathrm{Zn} \\
\mathrm{Zr} \\
\mathrm{U} \\
\mathrm{Pll} \\
\mathrm{Am}\end{array}$ & $\begin{array}{c}0.7 \\
<0.1 \\
0.6 \\
6.3 \\
0.4 \\
0.4 \\
1.0 \\
<0.1 \\
1.0 \\
2.6 \\
0.4 \\
1.8 \\
1.5 \\
1.8\end{array}$ & $\begin{array}{c}0.6 \\
<0.1 \\
0.6 \\
6.3 \\
0.4 \\
0.7 \\
0.7 \\
<0.1 \\
1.0 \\
2.8 \\
0.3 \\
1.7 \\
0.9 \\
1.8\end{array}$ & $\begin{array}{c}0.5 \\
<(0.1 \\
0.5 \\
01 \\
(0.3 \\
0.7 \\
0.6 \\
<(0.1 \\
0.9 \\
2.5 \\
0.3 \\
1.7 \\
0.8 \\
1.7\end{array}$ \\
\hline
\end{tabular}

"These solutions contained $60 \mathrm{mg} \mathrm{Pu} / \mathrm{L}$; all others contained $3 \mathrm{~g} \mathrm{Pu/L}$. 
d. JSK-2 Anion Exchange Resin. Plutonium sorbs well from acidic solution, while cerium, technetium, uranium, and americium sorb at lower levels. Only technetium sorbs at a useful level from basic solution.

Table III.B.2.d. Distribution of 14 Elements onto JSK-2 Experimental Anion Exchange Resin from Two Simulant Solutions for Hanford HLW Tank 102-SY

\begin{tabular}{|c|c|c|c|c|}
\hline Medium & Absorber & 30-min Kd & 2-h Kd & 6-h Kd \\
\hline \multirow[t]{17}{*}{ Acid-Dissolved Sludge } & $\mathrm{Ce}$ & 27 & 26 & 24 \\
\hline & Cs & 0.3 & 0.1 & 0.1 \\
\hline & $\mathrm{Sr}$ & 0.6 & 0.5 & 0.6 \\
\hline & $\mathrm{Tc}$ & 28 & 28 & 27 \\
\hline & $Y$ & 1.6 & 1.7 & 1.4 \\
\hline & $\mathrm{Cr}$ & 0.4 & 0.4 & 0.5 \\
\hline & Co & $<0.1$ & $<0.1$ & 0.1 \\
\hline & $\mathrm{Fe}$ & $<0.1$ & $<0.1$ & 0.3 \\
\hline & $\mathrm{Mn}$ & 0.3 & 0.1 & 0.1 \\
\hline & $\mathrm{Zn}$ & 2.8 & 1.9 & 1.1 \\
\hline & $\mathrm{Zr}$ & 5.5 & 5.6 & 5.8 \\
\hline & U & 19 & 19 & 19 \\
\hline & $\mathrm{U}^{\mathrm{a}}$ & 20 & 17 & 17 \\
\hline & $\mathrm{Pu}$ & 142 & 278 & 422 \\
\hline & $\mathrm{Pu}^{\mathrm{a}}$ & 329 & 337 & 261 \\
\hline & $\mathrm{Am}$ & 8.4 & 7.9 & 7.6 \\
\hline & $A m^{\prime \prime}$ & 9.4 & 7.7 & 7.2 \\
\hline \multirow[t]{14}{*}{ Alkaline Supernate } & $\mathrm{Ce}$ & 0.6 & - & 1.2 \\
\hline & Cs & 0.1 & - & 0.3 \\
\hline & $\mathrm{Sr}$ & 0.2 & - & 0.5 \\
\hline & $\mathrm{Tc}$ & 50 & - & 51 \\
\hline & $\mathrm{Y}$ & $<0.1$ & - & 0.5 \\
\hline & $\mathrm{Cr}$ & $<0.1$ & - & 0.6 \\
\hline & $\mathrm{Co}$ & 0.1 & - & 0.4 \\
\hline & $\mathrm{Fe}$ & $<0.1$ & 0.1 & 0.3 \\
\hline & $\mathrm{Mn}$ & 0.7 & 0.2 & 0.3 \\
\hline & $\mathrm{Zn}$ & 1.9 & 1.8 & 1.8 \\
\hline & $\mathrm{Zr}$ & $<0.1$ & - & 0.5 \\
\hline & $U$ & 1.3 & 1.2 & 1.2 \\
\hline & $\mathrm{Pu}$ & 0.2 & 0.3 & 0.1 \\
\hline & $\mathrm{Am}$ & 1.8 & 1.2 & 1.1 \\
\hline
\end{tabular}

"These solutions contained $60 \mathrm{mg} \mathrm{Pu} / \mathrm{L}$; all others contained $3 \mathrm{~g} \mathrm{Pu} / \mathrm{L}$. 
e. JSK-3 Anion Exchange Resin. The sorption of plutonium is significantly greater than on any other absorber from acidic solution, whereas the sorption of other elements from acidic and basic solution is similar to that of JSK-2 resin. The exceptionally high sorption of plutonium from acid exceeds that of the next best absorber, Reillex ${ }^{\mathrm{TM}}$ HPQ resin, by an order of magnitude.

Table III.B.2.e. Distribution of 14 Elements onto JSK-3 Experimental Anion Exchange Resin from Two Simulant Solutions for Hanford HLW Tank 102-SY

\begin{tabular}{|c|c|c|c|c|}
\hline Medium & Absorber & 30-min Kd & 2-h Kd & 6-h Kd \\
\hline \multirow[t]{17}{*}{ Acid-Dissolved Sludge } & $\mathrm{Ce}$ & 23 & 23 & 22 \\
\hline & Cs & 0.4 & 0.1 & 0.4 \\
\hline & $\mathrm{Sr}$ & 1.0 & 1.3 & 1.3 \\
\hline & $\mathrm{Tc}$ & 24 & 24 & 22 \\
\hline & $Y$ & 1.2 & 1.1 & 1.3 \\
\hline & $\mathrm{Cr}$ & 0.4 & 0.6 & 0.7 \\
\hline & $\mathrm{Co}$ & $<0.1$ & 0.3 & 0.1 \\
\hline & $\mathrm{Fe}$ & 0.4 & 0.1 & 0.3 \\
\hline & $\mathrm{Mn}$ & $<0.1$ & 0.2 & 0.4 \\
\hline & $\mathrm{Zn}$ & 1.7 & 1.3 & 0.7 \\
\hline & $\mathrm{Zr}$ & 3.7 & 3.3 & 3.0 \\
\hline & $U$ & 18 & 18 & 17 \\
\hline & $\mathrm{U}^{\mathrm{a}}$ & 18 & 17 & 17 \\
\hline & $\mathrm{Pu}$ & 1053 & 1035 & 1173 \\
\hline & $\mathrm{Pu}^{\mathrm{i}}$ & 870 & 1650 & 2185 \\
\hline & $\mathrm{Am}$ & 6.2 & 5.3 & 5.0 \\
\hline & $\mathrm{Am}^{a}$ & 7.9 & 6.3 & 6.0 \\
\hline \multirow[t]{14}{*}{ Alkaline Supernate } & $\mathrm{Ce}$ & 1.4 & 1.4 & 1.4 \\
\hline & Cs & $<0.1$ & $<0.1$ & 0.1 \\
\hline & $\mathrm{Sr}$ & 0.8 & 0.8 & 0.8 \\
\hline & $\mathrm{Tc}$ & 48 & 49 & 50 \\
\hline & $\mathrm{Y}$ & 0.6 & 0.7 & 0.5 \\
\hline & $\mathrm{Cr}$ & 0.0 & 0.5 & 0.6 \\
\hline & Co & 1.1 & 1.3 & 0.9 \\
\hline & $\mathrm{Fe}$ & $<0.1$ & $<0.1$ & $<0.1$ \\
\hline & $\mathrm{Mn}$ & 0.8 & 0.9 & 0.5 \\
\hline & $\mathrm{Zn}$ & 1.7 & 1.3 & 1.5 \\
\hline & $\mathrm{Zr}$ & 0.3 & 0.2 & 0.1 \\
\hline & $\mathrm{U}$ & 2.0 & 1.4 & 1.5 \\
\hline & $\mathrm{Pu}$ & 0.7 & 0.4 & 0.4 \\
\hline & Am & 1.7 & 1.3 & 1.1 \\
\hline
\end{tabular}


f. Crypt-DER Polymer Resin. Crypt-DER polymer resin is a novel absorber prepared by the Materials Technology: Polymers and Coatings Group at Los Alamos National Laboratory. The granular solid is obtained by polymerizing an octalazacryptand compound with a commercially available di-epoxide resin (DER). The cryptand functionality contained in the polymer selectively sorbs certain metal ions [e.g., $\mathrm{Hg}$ (II) and $\mathrm{Cd}(\mathrm{II})$ ] from solutions of $\mathrm{pH}>3$. However, when the amine nitrogens of the cryptand are protonated at a lower $\mathrm{pH}$, the cationic functionality binds fluoride ions quite selectively. We included the Crypt-DER polymer resin in this study to obtain additional information about its metal sorption properties.

Plutonium, technetium, and uranium sorb at useful levels from acidic solution, while cerium and zirconium are weakly sorbed. Only technetium offers useful Kds from basic solution.

Table III.B.2.f. Distribution of 14 Elements onto Crypt-DER Polymer Resin from Two Simulant Solutions for Hanford HLW Tank 102-SY

\begin{tabular}{|c|c|c|c|c|}
\hline Medium & Absorber & 30-min Kd & 2-h Kd & 6-h Kd \\
\hline \multirow[t]{14}{*}{ Acid-Dissolved Sludge } & $\mathrm{Ce}$ & 3.0 & 2.9 & 2.8 \\
\hline & Cs & 0.1 & 0.3 & 0.2 \\
\hline & $\mathrm{Sr}$ & 0.8 & 0.5 & 0.4 \\
\hline & $\mathrm{Tc}$ & 9.8 & 9.9 & 9.8 \\
\hline & $\mathrm{Y}$ & 1.0 & 0.7 & 0.6 \\
\hline & $\mathrm{Cr}$ & $<0.1$ & $<0.1$ & $<0.1$ \\
\hline & Co & 0.3 & 0.3 & 0.3 \\
\hline & $\mathrm{Fe}$ & $<0.1$ & $<0.1$ & 0.1 \\
\hline & $\mathrm{Mn}$ & 0.8 & 0.7 & 0.4 \\
\hline & $\mathrm{Zn}$ & 0.7 & 0.5 & 0.3 \\
\hline & $\mathrm{Zr}$ & 3.2 & 3.6 & 3.9 \\
\hline & $\mathrm{U}$ & 3.6 & 6.3 & 8.3 \\
\hline & $\mathrm{Pu}$ & 4.1 & 8.0 & 19 \\
\hline & $\mathrm{Am}$ & 1.0 & 0.9 & 1.0 \\
\hline \multirow[t]{14}{*}{ Alkaline Supernate } & $\mathrm{Ce}$ & 0.9 & 0.7 & 0.7 \\
\hline & Cs & 0.8 & 0.8 & 1.0 \\
\hline & $\mathrm{Sr}$ & 0.7 & 0.3 & 0.4 \\
\hline & $\mathrm{Tc}$ & 25 & 25 & 25 \\
\hline & Y & 1.0 & 0.6 & 0.8 \\
\hline & $\mathrm{Cr}$ & 0.5 & 0.1 & 0.1 \\
\hline & Co & 2.1 & 2.1 & 2.1 \\
\hline & $\mathrm{Fe}$ & 0.1 & 0.2 & 0.4 \\
\hline & $\mathrm{Mn}$ & 0.8 & 0.8 & 1.1 \\
\hline & $\mathrm{Zn}$ & 1.2 & 1.3 & 1.4 \\
\hline & $\mathrm{Zr}$ & $<0.1$ & 0.3 & 0.2 \\
\hline & $U$ & 1.6 & 1.7 & 1.9 \\
\hline & $\mathrm{Pu}$ & $<0.1$ & $<0.1$ & $<0.1$ \\
\hline & $\mathrm{Am}$ & 1.2 & 1.4 & 1.5 \\
\hline
\end{tabular}


g. PK-01-244 Phosphinic Acid Resin. This PK-01-244 phosphinic acid cation exchanger, prepared by Prof. Spiro Alexandratos at the University of Tennessee, wass stated to contain 45\% water. We followed the accompanying directions to alvoid drying this resin and took 455-mg portions that should have contained $250 \mathrm{mg}$ of the dry resin.

Only cesium and technetium exhibit a Kd consistently higher than I from either acidic or basic solution.

Table III.B.2.g. Distribution of 14 Elements onto PK-01-244 Resin from Two Simulant Solutions for Hanford HLW Tank 102-SY

\begin{tabular}{|c|c|c|c|c|}
\hline Medium & Absorber & 30-min Kd & 2-h Kd & 6-h Kd \\
\hline \multirow[t]{14}{*}{ Acid-Dissolved Sludge } & $\mathrm{Ce}$ & 0.8 & 0.8 & 0.5 \\
\hline & Cs & 1.0 & 0.9 & 0.5 \\
\hline & $\mathrm{Sr}$ & 0.6 & 0.6 & 0.5 \\
\hline & $\mathrm{Tc}$ & 1.5 & 1.7 & 1.9 \\
\hline & $Y$ & 0.6 & 0.8 & 0.6 \\
\hline & $\mathrm{Cr}$ & 0.6 & 0.6 & 0.4 \\
\hline & Co & 0.9 & 0.7 & 0.5 \\
\hline & $\mathrm{Fe}$ & 0.7 & 0.2 & 0.2 \\
\hline & $\mathrm{Mn}$ & 1.0 & 0.3 & 0.3 \\
\hline & $\mathrm{Zn}$ & 0.8 & 0.4 & 0.4 \\
\hline & $\mathrm{Zr}$ & 1.1 & 0.6 & 1.0 \\
\hline & U & $<0.1$ & 0.3 & 0.3 \\
\hline & $\mathrm{Pu}$ & 0.6 & 0.3 & 0.2 \\
\hline & $\mathrm{Am}$ & 0.8 & 0.4 & 0.3 \\
\hline \multirow{14}{*}{ Alkaline Supernate } & $\mathrm{Ce}$ & 0.5 & 0.5 & 0.8 \\
\hline & Cs & 1.4 & 1.4 & 1.7 \\
\hline & $\mathrm{Sr}$ & 0.3 & 0.3 & 0.7 \\
\hline & $\mathrm{Tc}$ & 0.9 & 0.8 & 1.4 \\
\hline & $Y$ & 0.7 & 0.4 & 0.7 \\
\hline & $\mathrm{Cr}$ & $<0.1$ & 0.1 & 0.1 \\
\hline & Co & 0.7 & 0.4 & 1.0 \\
\hline & $\mathrm{Fe}$ & 0.2 & 0.1 & 0.1 \\
\hline & $\mathrm{Mn}$ & 0.5 & 0.3 & 0.3 \\
\hline & $\mathrm{Zn}$ & 0.2 & 0.2 & 0.2 \\
\hline & $\mathrm{Zr}$ & 0.5 & 0.1 & 0.1 \\
\hline & $\mathrm{U}$ & 0.5 & 0.4 & 0.5 \\
\hline & $\mathrm{Pu}$ & 0.3 & 0.3 & 0.2 \\
\hline & $\mathrm{Am}$ & $<0.1$ & $<0.1$ & 0.1 \\
\hline
\end{tabular}


h. SRS Formaldehyde/Kesorcinol Resin. Jane Bibler of Savannah River Site (SRS), who provided this formaldehyde/resorcinol resin, recommended that we convert it from the asreceived potassium form to a sodium form. This was done by passing ten volumes of $10 \% \mathrm{NaOH}$ solution through a column of potassium-form resin, followed by 15 column volumes of water, after which the resin was air-dried. The air-dried weight, per water-wet $\mathrm{ml}$, wals $0.254 \mathrm{~g}$.

Chromium, technetium, and cesium sorb somewhat from acidic solution. Cesium sorbs well from basic solution, but is accompanied by significant levels of many other clements.

Table III.B.2.h. Distribution of 14 Elements onto SRS Formaldehyde/Resorcinol Resin from Two Simulant Solutions for Hanford HLW Tank 102-SY

\begin{tabular}{|c|c|c|c|c|}
\hline Medium & Absorber & 30-min Kd & 2-h Kd & 6-h Kd \\
\hline \multirow[t]{14}{*}{ Acid-Dissolved Sludge } & $\mathrm{Ce}$ & 0.8 & 1.0 & 1.1 \\
\hline & $\mathrm{Cs}$ & 2.7 & 4.5 & 5.9 \\
\hline & $\mathrm{Sr}$ & 1.2 & 0.8 & 1.1 \\
\hline & $\mathrm{Tc}$ & 2.7 & 4.5 & 11 \\
\hline & $\mathrm{Y}$ & 0.7 & 1.0 & 1.1 \\
\hline & $\mathrm{Cr}$ & 0.8 & 0.9 & 1.0 \\
\hline & Co & 1.0 & 1.2 & 1.3 \\
\hline & $\mathrm{Fe}$ & 0.2 & 0.5 & 0.3 \\
\hline & $\mathrm{Mn}$ & 0.3 & 0.4 & 0.5 \\
\hline & $\mathrm{Zn}$ & 0.2 & 0.5 & 0.5 \\
\hline & $\mathrm{Zr}$ & 1.3 & 0.9 & 1.5 \\
\hline & $\mathrm{U}$ & 0.2 & $<0.1$ & 0.3 \\
\hline & $\mathrm{Pu}$ & $<0.1$ & 0.1 & 0.1 \\
\hline & $\mathrm{Am}$ & $<0.1$ & 0.4 & 0.1 \\
\hline \multirow[t]{14}{*}{ Alkaline Supernate } & $\mathrm{Ce}$ & 14 & 26 & 42 \\
\hline & $\mathrm{Cs}$ & 75 & 85 & 66 \\
\hline & $\mathrm{Sr}$ & 4.8 & 2.6 & 1.5 \\
\hline & $\mathrm{Tc}$ & 1.0 & 1.5 & 1.8 \\
\hline & $Y$ & 12 & 21 & 36 \\
\hline & $\mathrm{Cr}$ & 0.3 & 1.0 & 0.8 \\
\hline & $\mathrm{Co}$ & 12 & 15 & 7.7 \\
\hline & $\mathrm{Fe}$ & 0.2 & 0.5 & 0.7 \\
\hline & $\mathrm{Mn}$ & 5.9 & 4.7 & 2.2 \\
\hline & $\mathrm{Zn}$ & 9.3 & 11 & 9.0 \\
\hline & $\mathrm{Zr}$ & $<0.1$ & $<0.1$ & 0.1 \\
\hline & U & 7.5 & 13 & 22 \\
\hline & $\mathrm{Pu}$ & 0.2 & 0.9 & 1.2 \\
\hline & $\mathrm{Am}$ & 11 & 19 & 27 \\
\hline
\end{tabular}


3. Polyacrylonitrile (PAN) Composite Absorbers. Although inorganic ion exchangers are known to offer high selectivity, high capacity, and rapid kinetics, their granular and mechanical properties often make them unsuitable for column applications. One way to circumvent these limitations is to incorporate the inorganic exchange materials in beads of a suitable porous polymer. ${ }^{12}$

Nine composite absorbers were prepared and provided to us by Dr. Ferdinand Sebesta of the Czech Technical University in Prague. These composites consist of various inorganic exchange materials dispersed in modified-binding-polymer beads of polyacrylonitrile (PAN).

a. TiO-PAN Titanium Dioxide Composite. The titanium dioxide content of the dry TiO-PAN composite was $85 \%$. The air-dried weight of this composite, per water-wet ml, wals $0.231 \mathrm{~g}$.

Zirconium is strongly sorbed from acidic solution, albiet with slow kinetics; only plutonium offers even weak competition. Uranium is strongly sorbed from basic solution, while several other elements are slightly sorbed.

Table III.B.3.a. Distribution of 14 Elements onto TiO-PAN Composite Resin from Two Simulant Solutions for Hanford HLW Tank 102-SY

\begin{tabular}{|c|c|c|c|c|}
\hline Medium & Absorber & 30-min Kd & 2-h Kd & 6-h Kd \\
\hline \multirow[t]{14}{*}{ Acid-Dissolved Sludge } & $\mathrm{Ce}$ & 0.7 & 0.4 & 0.5 \\
\hline & Cs & ().3 & 0.1 & ().4 \\
\hline & $\mathrm{Sr}$ & 1.1 & 0.4 & 0.8 \\
\hline & $\mathrm{Tc}$ & 1.0 & 0.7 & 0.9 \\
\hline & $\mathrm{Y}$ & 0.8 & 0.6 & 0.6 \\
\hline & $\mathrm{Cr}$ & 0.5 & $(0.4$ & 0.5 \\
\hline & $\mathrm{Co}$ & 0.6 & 0.3 & 0.4 \\
\hline & $\mathrm{Fe}$ & 0.4 & 0.4 & 0.8 \\
\hline & $\mathrm{Mn}$ & 0.1 & 0.1 & 0.2 \\
\hline & $\mathrm{Zn}$ & $<0.1$ & 0.2 & 0.3 \\
\hline & $\mathrm{Zr}$ & 51 & 323 & 899 \\
\hline & $\mathrm{U}$ & 0.3 & 0.3 & 0.6 \\
\hline & $\mathrm{Pu}$ & 6.3 & 7.7 & 8.6 \\
\hline & Am & 0.1 & $<0.1$ & $<() .1$ \\
\hline \multirow[t]{14}{*}{ Alkaline Supernate } & $\mathrm{Ce}$ & 1.6 & 1.9 & 1.6 \\
\hline & Cs & 0.6 & 1.0 & 0.6 \\
\hline & $\mathrm{Sr}$ & 0.2 & 0.5 & 0.4 \\
\hline & $\mathrm{Tc}$ & 1.3 & 1.5 & 1.6 \\
\hline & $\mathrm{Y}$ & 1.5 & 2.0 & 1.8 \\
\hline & $\mathrm{Cr}$ & 4.0 & 4.4 & 5.4 \\
\hline & $\mathrm{Co}$ & $<() .1$ & $<0.1$ & $<0.1$ \\
\hline & $\mathrm{Fe}$ & 1.4 & 3.8 & 5.2 \\
\hline & $\mathrm{Mn}$ & $<0.1$ & $<0.1$ & 0.1 \\
\hline & $\mathrm{Zn}$ & $<0.1$ & 0.2 & 0.1 \\
\hline & $\mathrm{Zr}$ & 1.5 & 2.0 & 3.2 \\
\hline & $\mathrm{U}$ & 67 & 115 & 185 \\
\hline & $\mathrm{Pu}$ & 1.1 & 1.5 & 2.0 \\
\hline & $\mathrm{Am}$ & 2.1 & 2.8 & 3.2 \\
\hline
\end{tabular}


b. NiFC.PAN Nickel Ferrocyanide Composite. The nickel ferrocyanide content of the dry NiFC-PAN composite was $85 \%$. The air-dried weight of this composite, per waler-wet ml, was $0.231 \mathrm{~g}$.

This absorber appears to be selective for cesium from acidic solution. From basic solution. cesium sorbed strongly, zinc is well sorbed, cobalt is moderately sorbed, and uranium and iron are slightly sorbed.

Table III.B.3.b. Distribution of 14 Elements onto NiFC.PAN Composite Resin from Two Simulant Solutions for Hanford HLW Tank 102-SY

\begin{tabular}{|c|c|c|c|c|}
\hline Medium & Absorber & 30-min Kd & $2 \cdot h \mathrm{Kd}$ & 6-h Kd \\
\hline \multirow[t]{14}{*}{ Acid-Dissolved Sludge } & $\mathrm{Ce}$ & 0.5 & $(0.3$ & 0.5 \\
\hline & $\mathrm{Cs}$ & 45 & 49 & 49 \\
\hline & $\mathrm{Sr}$ & 0.5 & 0.6 & 0.7 \\
\hline & $\mathrm{Tc}$ & 0.4 & 0.8 & 0.7 \\
\hline & $\mathrm{Y}$ & 0.5 & 0.7 & 0.6 \\
\hline & $\mathrm{Cr}$ & 0.5 & 0.5 & 0.6 \\
\hline & Co & 1.1 & 1.1 & 1.1 \\
\hline & $\mathrm{Fe}$ & 0.4 & 0.3 & 0.5 \\
\hline & $\mathrm{Mn}$ & 0.3 & 0.3 & 0.4 \\
\hline & $\mathrm{Zn}$ & 0.3 & 0.4 & 0.4 \\
\hline & $\mathrm{Zr}$ & 1.0 & 1.1 & 1.7 \\
\hline & $\mathrm{U}$ & 0.6 & 0.4 & 0.2 \\
\hline & $\mathrm{Pu}$ & 0.2 & 0.7 & 0.4 \\
\hline & $\mathrm{Am}$ & $<0.1$ & $<0.1$ & 0.1 \\
\hline \multirow[t]{14}{*}{ Alkaline Supernate } & $\mathrm{Ce}$ & 0.3 & 0.3 & 0.2 \\
\hline & Cs & 3533 & 11954 & 5998 \\
\hline & $\mathrm{Sr}$ & $<0.1$ & $<0.1$ & $<0.1$ \\
\hline & $\mathrm{Tc}$ & 1.0 & 0.7 & 0.8 \\
\hline & $\mathrm{Y}$ & $<0.1$ & 0.2 & 0.2 \\
\hline & $\mathrm{Cr}$ & 0.9 & 0.8 & 1.2 \\
\hline & $\mathrm{Co}$ & 8.9 & 10 & 11 \\
\hline & $\mathrm{Fe}$ & 1.4 & 1.7 & 2.3 \\
\hline & $\mathrm{Mn}$ & 0.5 & 0.7 & 0.5 \\
\hline & $\mathrm{Zn}$ & 61 & 94 & 90 \\
\hline & $\mathrm{Zr}$ & 0.5 & 0.5 & 0.7 \\
\hline & $\mathrm{U}$ & 2.1 & 2.2 & 2.0 \\
\hline & $\mathrm{Pu}$ & $<0.1$ & $<0.1$ & 0.2 \\
\hline & $\mathrm{Am}$ & 0.4 & 0.3 & 0.2 \\
\hline
\end{tabular}


c. Mg()-PAN Magnesium (Oxide Composite. The magnesium oxide content of the dry MgO-PAN composite was $80 \%$. The air-dried weight of this composite, per water-wet ml, was $0.226 \mathrm{~g}$.

Zirconium and several other elements are slightly sorbed from acidic solution. Beciuse this absorber was unstable in basic solution, distributions in basic solution could non be measured. The observed resin swelling and solution discoloration indicalled some instability, even in acidic solution.

Table III.B.3.c. Distribution of 14 Elements onto MgO-PAN Composite Resin from Acid-Dissolved Sludge Simulant for Hanford HLW Tank 1(22-SY

\begin{tabular}{lcccc}
\hline Medium & Absorber & 30-min Kd & 2-h Kd & 6-h Kd \\
\hline Acid-Dissolved Sludge & $\mathrm{Ce}$ & 1.5 & 1.5 & 1.5 \\
& $\mathrm{Cs}$ & 0.8 & 1.1 & 1.1 \\
& $\mathrm{Sr}$ & 1.4 & 1.6 & 2.0 \\
& $\mathrm{Tc}$ & 1.4 & 1.7 & 1.9 \\
& $\mathrm{Y}$ & 1.2 & 1.5 & 1.5 \\
& $\mathrm{Cr}$ & 1.5 & 1.4 & 1.6 \\
& $\mathrm{Co}$ & 1.3 & 1.4 & 1.4 \\
& $\mathrm{Fe}$ & 0.7 & 0.8 & 1.0 \\
& $\mathrm{Mn}$ & 0.3 & 0.3 & 0.3 \\
& $\mathrm{Zn}$ & 0.4 & 0.4 & 0.4 \\
& $\mathrm{Zr}$ & 2.2 & 3.2 & 4.7 \\
& $\mathrm{U}$ & 0.1 & 0.3 & 0.5 \\
& $\mathrm{Pu}$ & 0.9 & 1.3 & 1.6 \\
& $\mathrm{Am}$ & 0.3 & 0.3 & 0.3 \\
\hline
\end{tabular}


d. Mn().PAN Mungunese Dloxide Composite. The manganese diuxide comlent of the diy

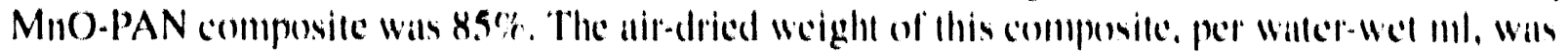
$0.210 \mathrm{~g}$.

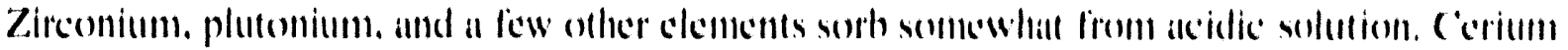
and americium show a more than lenfold higher sorption from basic solution han yerrium. lranium, chromium, and iron are moderincly sorbed from basic solution.

Table III.13.3.d. Distribution of 14 Elements onto MnO-PAN (omponile Resin lirom Tiwo Simulant Solutions for Hunford HLW Tank 1()2-SY

\begin{tabular}{|c|c|c|c|c|}
\hline Medium & Absorber & $3(1) \cdot \mathrm{m} \ln \mathrm{Kd}$ & $2 \cdot h \mathrm{Kd}$ & 6-h Ka \\
\hline \multirow[t]{14}{*}{ Acid-Dissolved Sludge } & $\mathrm{Ce}$ & 1.1 & 1.0 & $(1) .9$ \\
\hline & Cs & 1.2 & 1.2 & 1.1 \\
\hline & $\mathrm{Sr}$ & 0.9 & 0.0 & (1).6 \\
\hline & $T c$ & 1.2 & 1.3 & 1.0 \\
\hline & $Y$ & 1.1 & 1.1 & 0.8 \\
\hline & $\mathrm{Cr}$ & 0.6 & $(0.9$ & 0.6 \\
\hline & Co & 0.9 & 0.8 & 0.7 \\
\hline & $\mathrm{Fe}$ & 0.7 & 1.2 & 1.0 \\
\hline & $\mathrm{Mn}$ & 0.3 & 0.3 & 0.5 \\
\hline & $\mathrm{Zn}$ & $<0.1$ & 0.2 & $(0.2$ \\
\hline & $\mathrm{Zr}$ & 4.0 & 6.0 & 6.8 \\
\hline & U & 0.5 & 0.1 & 0.5 \\
\hline & $\mathrm{Pu}$ & 2.5 & 2.7 & 2.8 \\
\hline & $A m$ & 0.2 & 0.2 & 0.1 \\
\hline \multirow[t]{14}{*}{ Alkaline Supernate } & $\mathrm{Co}$ & 24 & 2.5 & 26 \\
\hline & $\mathrm{Cs}$ & 0.5 & 0.8 & 1.1 \\
\hline & $\mathrm{Sr}$ & $<0.1$ & $<0.1$ & $<() .1$ \\
\hline & $T c$ & 1.1 & 1.4 & 1.4 \\
\hline & $\mathrm{Y}$ & 2.0 & 2.1 & 1.8 \\
\hline & $\mathrm{Cr}$ & 3.0 & 4.3 & 4.8 \\
\hline & $\mathrm{Co}$ & $<() .1$ & 0.6 & $(0.9$ \\
\hline & $\mathrm{Fc}$ & 3.1 & 4.5 & 5.8 \\
\hline & $\mathrm{Mn}$ & 1.8 & 1.9 & 2.2 \\
\hline & $\mathrm{Zn}$ & $<() .1$ & 0.1 & ().2 \\
\hline & $\mathrm{Zr}$ & 1.7 & 2.2 & 2.3 \\
\hline & $U$ & 11 & 1.3 & 1.3 \\
\hline & $\mathrm{Pu}$ & 2.8 & 3.3 & 3.8 \\
\hline & Am & 32 & 30 & 20 \\
\hline
\end{tabular}




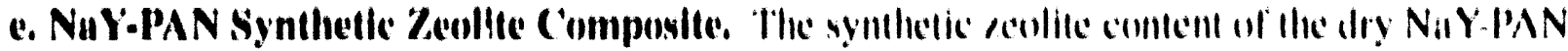

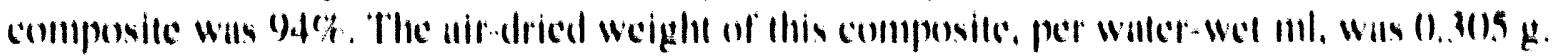

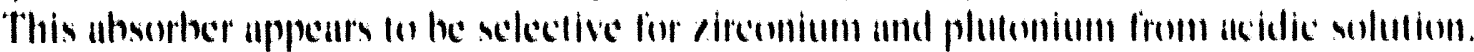

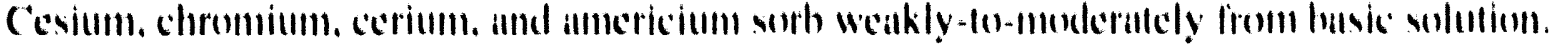

Tuble III.B.3.e. Distribution of It Elements onto NaY.PAN Componsite Resin from Tiwo Simulant Solutions for Hanford HI.W Tank I(12.SY

\begin{tabular}{|c|c|c|c|c|}
\hline Medlum & Absorber & 3).min Kd & 2.h Kd & 6-h Kd \\
\hline \multirow[t]{14}{*}{ Acid-Dissulved Sluclge } & $\mathrm{Cc}$ & 0.9 & 1.1 & 10.9 \\
\hline & $\mathrm{Cs}$ & 0.9 & 1.0 & 0.7 \\
\hline & $\mathrm{Sr}$ & 0.7 & 0.9 & 0.9 \\
\hline & $\mathrm{Tc}$ & 1.0 & 0.9 & 0.9 \\
\hline & $Y$ & 0.9 & 1.0 & 0.9 \\
\hline & $\mathrm{Cr}$ & 0.9 & 1.0 & $(0.9$ \\
\hline & Co & 0.8 & 0.9 & 0.9 \\
\hline & $\mathrm{Fc}$ & 2.2 & 1.0 & 1.5 \\
\hline & $M_{n}$ & 0.4 & 0.1 & (0.1 \\
\hline & $\mathrm{Zn}$ & (0.2 & $(0.2$ & 0.1 \\
\hline & $\mathrm{Zr}$ & 107 & 191 & 108 \\
\hline & 11 & 0.3 & 0.1 & $(0.3$ \\
\hline & $P u$ & 12 & 1.5 & 1.5 \\
\hline & Am & $<0.1$ & $<() .1$ & $<() .1$ \\
\hline \multirow[t]{14}{*}{ Alkuline Supernalle } & $\mathrm{Co}$ & 4.6 & 4.0 & $3 . .3$ \\
\hline & $C s$ & 11 & 10 & 10 \\
\hline & $\mathrm{Sr}$ & 0.6 & 1.0 & 0.9 \\
\hline & $T c$ & 0.1 & 0.5 & ()..3 \\
\hline & $Y$ & 1.5 & 1.6 & 1.4 \\
\hline & $\mathrm{Cr}$ & 2.3 & 4.1 & 4.8 \\
\hline & $\mathrm{Co}$ & 0.7 & 0.4 & (0.4 \\
\hline & $\mathrm{Fo}$ & (). 2 & 0.5 & 0.9 \\
\hline & $M_{n}$ & 0.8 & 0.7 & (1). 7 \\
\hline & $\mathrm{Zn}$ & 1.5 & 1.3 & 1.2 \\
\hline & $\mathrm{Zr}$ & 0.2 & $(0.8$ & $(0.8$ \\
\hline & $U$ & 0.9 & 0.8 & (1).9 \\
\hline & $P_{u}$ & $<() .1$ & $<() .1$ & $<() .1$ \\
\hline & Am & 3.7 & 3.5 & 3.3 \\
\hline
\end{tabular}




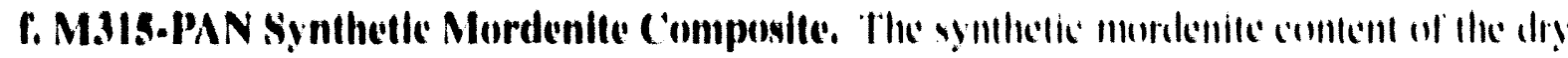

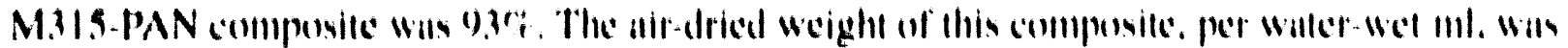
$11.222 \mathrm{~g}$.

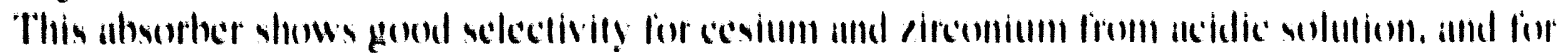
cesilum lrom basice sulution.

Thable III.1B.3.f. Distribution of It Elemenls ontu M.315.PAN (amposile Resin from Two Simulant Solurions for Hanlord HI.W Tiunk 1(12.SY

\begin{tabular}{|c|c|c|c|c|}
\hline Medium & Absorber & 3).min Kd & $2 \cdot h \mathrm{Kd}$ & h-h Kd \\
\hline \multirow[t]{14}{*}{ Acid-Dissulved Sludge } & Co & 0.9 & 1.11 & 0.8 \\
\hline & Cs & 5() & 04 & 01 \\
\hline & $\mathrm{Sr}$ & $(0.8$ & 1.0 & 0.9 \\
\hline & $\mathrm{Tc}$ & 0.7 & 0.7 & 0.8 \\
\hline & $Y$ & 1.11 & 1.0 & 0.8 \\
\hline & $\mathrm{Cr}$ & 0.8 & $1.1)$ & 0.7 \\
\hline & Co & 1.3 & 1.0 & 1.1 \\
\hline & Fe & 0.8 & (1).5 & 0.5 \\
\hline & $M n$ & 0.3 & 0.2 & 0.2 \\
\hline & $\mathrm{Zn}$ & 0.3 & 0.4 & 0.3 \\
\hline & $\mathrm{Zr}$ & 27 & 67 & 96 \\
\hline & 11 & 0.4 & 0.6 & 0.4 \\
\hline & $P u$ & 1.1 & $1 .(1)$ & 1.2 \\
\hline & Am & $<() .1$ & $<() .1$ & $<(1) .1$ \\
\hline \multirow[t]{14}{*}{ Alkuline Supernute } & $\mathrm{Ce}$ & 0.5 & 0.2 & 0.4 \\
\hline & $\mathrm{Cs}$ & 191 & 227 & 2.31 \\
\hline & $\mathrm{Sr}$ & 1.1 & 1.2 & 1.4 \\
\hline & $\mathrm{Tc}$ & 0.3 & 0.6 & ().6 \\
\hline & $Y$ & 0.6 & 0.6 & (1).7 \\
\hline & $\mathrm{Cr}$ & 0.6 & 0.7 & $1 .(1)$ \\
\hline & Co & 0.1 & $<() .1$ & $<() .1$ \\
\hline & $\mathrm{Fc}$ & 0.5 & 0.7 & (). 7 \\
\hline & $M n$ & 0.6 & 0.6 & 0.6 \\
\hline & $\mathrm{Zn}$ & 0.5 & (0.2 & 0.4 \\
\hline & $\mathrm{Zr}$ & $<() .1$ & 0.3 & 0.5 \\
\hline & $U$ & 1.2 & 0.7 & $(1) .9$ \\
\hline & $P u$ & $<() .1$ & $<(0,1$ & $<() .1$ \\
\hline & Am & 0.8 & 0.6 & 0.7 \\
\hline
\end{tabular}




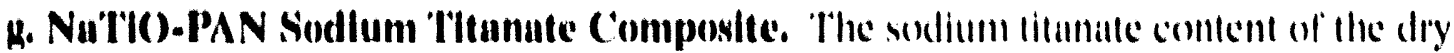
Natio-PAN composite was 92\%. The alr-dried weight of this composite, per willer-wet ml, was $11.324 \mathrm{~g}$.

Zirconium is moderallely sorbed and cesium is weakly sorthed from acidic solution. Zinc is well surbed from basic solution, whereas many other clements are moderallely or weakly sorhed.

Tuble III.B.3.y. Distribution of If Elements onto NaTiO-PAN Composite Resin lirom Two Simulant Solutions for Hanford HL.W Tank 1(12-SY

\begin{tabular}{|c|c|c|c|c|}
\hline Medlum & Absorber & $3(1)-m \ln K d$ & 2.h Kd & 6-h Kd \\
\hline \multirow[t]{14}{*}{ Acid-Dissolved Sludge } & $\mathrm{Co}$ & 0.9 & 0.5 & 0.8 \\
\hline & Cs & 2.2 & 1.6 & 1.9) \\
\hline & $\mathrm{Sr}$ & 0.5 & 0.0 & 0.7 \\
\hline & $T c$ & 0.9 & 0.0 & 1.1 \\
\hline & $\mathrm{Y}$ & 1.2 & 0.7 & 0.9 \\
\hline & $\mathrm{Cr}$ & 0.7 & 0.4 & 0.7 \\
\hline & Co & 0.9 & 0.6 & 0.7 \\
\hline & $\mathrm{Fc}$ & 0.7 & 0.3 & 0.4 \\
\hline & $\mathrm{Mn}$ & 0.4 & 0.4 & 0.4 \\
\hline & $7 . n$ & 0.4 & 0.4 & 0.4 \\
\hline & $\mathrm{Zr}$ & 3.5 & 5.4 & 1.3 \\
\hline & $U$ & 0.2 & 0.7 & 0.7 \\
\hline & PII & 0.9 & 0.8 & 1.0 \\
\hline & Am & $<() .1$ & $<0.1$ & $<() .1$ \\
\hline \multirow[t]{14}{*}{ Alkalline Supermute } & $\mathrm{Co}$ & 3.7 & 5.2 & 6.6 \\
\hline & Cs & 11 & 12 & 12 \\
\hline & $\mathrm{Sr}$ & 4.9 & 5.3 & 5.0 \\
\hline & $T s$ & 0.7 & 0.4 & 0.5 \\
\hline & $Y$ & 3.1 & 3.5 & 4.3 \\
\hline & $\mathrm{Cr}$ & 0.8 & 0.7 & 1.0) \\
\hline & (o) & 0.2 & 7.9 & 9.5 \\
\hline & $\mathrm{Fo}$ & 0.5 & 0.6 & 1.0 \\
\hline & $\mathrm{Mn}$ & $6.1)$ & 7.3 & 7.6 \\
\hline & $Z n$ & 21 & 4.5 & 71 \\
\hline & $7 \mathrm{r}$ & $<() .1$ & $<0.1$ & 0.2 \\
\hline & U & 4.5 & 0.8 & 8.2 \\
\hline & $P \|$ & $<() .1$ & $<() .1$ & 0.1 \\
\hline & AllI & 4.6 & 6.0) & 0.9 \\
\hline
\end{tabular}


h. Ba(Cu)SO)-PAN Burlum/Calclum Sulfute Composite. The harium/cialcium sulfitc content of the dry $\mathrm{Ba}(\mathrm{Ca}) \mathrm{SO}_{4}-\mathrm{PAN}$ composite was $8.5 \%$. The air-dered weight of this composite. per waller-wet ml, wass $0.198 \mathrm{~g}$.

Several elements are weakly sorbed from acidic solution. but none at useful levels. Strontium and cerium are moderately sorbed from basic solution.

Tuble Ill.B.3.h. Distribution of 14 Elements onto Ba(Ca)SO - PAN Composite Resin from Two Simulant Solutions for Hanford HLW Tank 1()2-SY

\begin{tabular}{|c|c|c|c|c|}
\hline Medium & Absorber & 30)-min Kd & 2.h Kd & 6-h Kd \\
\hline \multirow{14}{*}{ Acid-Dissolved Sludge } & $\mathrm{Ce}$ & 0.4 & 0.4 & 0.3 \\
\hline & Cs & 1.1 & 1.6 & 1.1 \\
\hline & $\mathrm{Sr}$ & 1.5 & 1.7 & 1.5 \\
\hline & $T c$ & 1.0 & 1.1 & 1.0 \\
\hline & $Y$ & 0.8 & 0.7 & 0.9 \\
\hline & $\mathrm{Cr}$ & 1.1 & 0.9 & 0.9 \\
\hline & $C_{0}$ & 1.0 & 0.9 & 0.8 \\
\hline & $\mathrm{Fe}$ & $<0.1$ & 0.2 & 0.2 \\
\hline & $\mathrm{Mn}$ & 0.3 & 0.1 & 0.1 \\
\hline & $\mathrm{Zn}$ & 0.2 & 0.2 & 0.2 \\
\hline & $\mathrm{Zr}$ & 1.0 & 2.0 & 1.9 \\
\hline & U & 0.4 & 0.5 & 0.4 \\
\hline & $\mathrm{Pu}$ & 0.9 & 0.9 & 0.7 \\
\hline & $A m$ & 0.1 & $<0.1$ & 0.1 \\
\hline \multirow[t]{14}{*}{ Alkuline Supernute } & $\mathrm{Cc}$ & 5.4 & 6.1 & 8.6 \\
\hline & $\mathrm{Cs}$ & 0.3 & 0.1 & 0.2 \\
\hline & $\mathrm{Sr}$ & 7.3 & 8.0 & 12 \\
\hline & $T c$ & 0.6 & 1.0 & 1.2 \\
\hline & $Y$ & 0.8 & 0.3 & 0.3 \\
\hline & $\mathrm{Cr}$ & $<0.1$ & $<0.1$ & 0.2 \\
\hline & $C_{0}$ & 0.4 & 0.3 & 0.2 \\
\hline & $\mathrm{Fc}$ & 0.5 & 0.7 & 0.9 \\
\hline & $\mathrm{Mn}$ & $<0.1$ & 0.1 & $<0.1$ \\
\hline & $\mathrm{Zn}$ & 0.4 & 0.1 & 0.2 \\
\hline & $\mathrm{Zr}$ & 0.7 & 0.7 & 0.8 \\
\hline & U & 1.2 & 1.7 & 2.2 \\
\hline & $\mathrm{Pu}$ & 0.8 & 0.5 & 1.0 \\
\hline & Am & 0.8 & 0,6 & 1.3 \\
\hline
\end{tabular}


I. AMP-PAN Ammonium Molybdophosphate Composite. The ammononium

molybdophosphate content of the dry composite was $81 \%$. The air-dried weight of this componite, per water-wet ml, was $0.23 .5 \mathrm{~g}$.

Cesium is strongly and selectively sorbed from acidic solution. (exitum also is strongly sorbed from basic solution, but is accompanied by lanthanides and annericium.

Table III.B.3.I. Distribution of 14 Elements onto AMP-PAN Composite Resin from Two Simulant Solutions for Hanford HLW Tank 1(02-SY

\begin{tabular}{|c|c|c|c|c|}
\hline Medium & Absorber & 3()-min Kd & 2.h Kd & 6.h Kd \\
\hline \multirow[t]{14}{*}{ Acid-Dissolved Sludge } & $\mathrm{Ce}$ & 0.4 & 0.2 & 0.4 \\
\hline & Cs & 1626 & 4072 & 55() 4 \\
\hline & $\mathrm{Sr}$ & 0.5 & 0.7 & 0.9 \\
\hline & $\mathrm{Tc}$ & 0.8 & 0.9 & 1.2 \\
\hline & $\mathrm{Y}$ & 0.8 & 0.5 & 0.4 \\
\hline & $\mathrm{Cr}$ & 1.0 & 0.9 & 1.0 \\
\hline & Co & 0.8 & 0.8 & 0.8 \\
\hline & $\mathrm{Fe}$ & 0.1 & 0.1 & 0.1 \\
\hline & $\mathrm{Mn}$ & 0.2 & 0.1 & 0.4 \\
\hline & $\mathrm{Zn}$ & 0.2 & 0.1 & 0.2 \\
\hline & $\mathrm{Zr}$ & 1.4 & 2.9 & 4.7 \\
\hline & U & 0.2 & 0.1 & 0.6 \\
\hline & $\mathrm{Pu}$ & 0.3 & 0.4 & 1.0 \\
\hline & $\mathrm{Am}$ & $<0.1$ & $<0.1$ & $<0.1$ \\
\hline \multirow{14}{*}{ Alkaline Supernate } & $\mathrm{Ce}$ & 12 & 22 & 28 \\
\hline & Cs & 2133 & 46.36 & 8275 \\
\hline & $\mathrm{Sr}$ & $<0.1$ & $<0.1$ & $<(0.1$ \\
\hline & $\mathrm{Tc}$ & 0.8 & 1.2 & 1.4 \\
\hline & Y & 15 & 27 & 34 \\
\hline & $\mathrm{Cr}$ & 0.5 & 1.3 & 1.3 \\
\hline & Co & 1.5 & 1.8 & 1.7 \\
\hline & $\mathrm{Fe}$ & 0.5 & 1.1 & 1.0 \\
\hline & $\mathrm{Mn}$ & $<0 . i$ & 0.1 & $<(0.1$ \\
\hline & $\mathrm{Zn}$ & 0.5 & 0.4 & 0.5 \\
\hline & $\mathrm{Zr}$ & 0.4 & 1.1 & 0.9 \\
\hline & U & 1.6 & 2.1 & 2.5 \\
\hline & $\mathrm{Pu}$ & 0.5 & 0.8 & 1.2 \\
\hline & $\mathrm{Am}$ & 24 & 50 & 86 \\
\hline
\end{tabular}


4. Phenolsulfonic-Formaldehyde Composite Absorbers. These two composites were prepared and provided to us by Dr. Jerzy Narbutt, Head, Department of Radiochemistry, Institute of Nuclear Chemistry and Technology, Warsaw, Poland. In these composite resins, the inorganic exchanger is dispersed in a matrix of phenolsulfonic-formaldehyde. ${ }^{13}$

a. Titanium Hexacyanoferrate Composite. The titanium hexacyanoferrate content of this composite was approximately $17 \%$.

Cesium is weakly sorbed from acidic solution. Cesium is more strongly sorbed from basic solution, although the sorption kinetics appear slow. Several other elements are moderately or weakly sorbed from basic solution.

Table III.B.4.a. Distribution of 14 Elements onto Titanium Hexacyanoferrate Composite Resin from Two Simulant Solutions for Hanford HLW Tank 102-SY

\begin{tabular}{|c|c|c|c|c|}
\hline Medium & Absorber & $30-\min \mathrm{Kd}$ & 2-h Kd & 6-h Kd \\
\hline \multirow[t]{14}{*}{ Acid-Dissolved Sludge } & $\mathrm{Ce}$ & $<0.1$ & 0.3 & 0.2 \\
\hline & $\mathrm{Cs}$ & 1.8 & 2.7 & 4.7 \\
\hline & $\mathrm{Sr}$ & 0.3 & 0.8 & 0.9 \\
\hline & $\mathrm{Tc}$ & 0.3 & 0.4 & 0.6 \\
\hline & $Y$ & 0.2 & 0.3 & 0.4 \\
\hline & $\mathrm{Cr}$ & 0.7 & 0.9 & 0.9 \\
\hline & $\mathrm{Co}$ & 0.6 & 0.7 & 0.8 \\
\hline & $\mathrm{Fe}$ & 0.4 & 0.3 & 0.3 \\
\hline & $\mathrm{Mn}$ & 0.4 & 0.3 & 0.5 \\
\hline & $\mathrm{Zn}$ & 0.4 & 0.2 & 0.5 \\
\hline & $\mathrm{Zr}$ & 0.8 & 0.8 & 1.7 \\
\hline & $\mathrm{U}$ & $<0.1$ & 0.2 & 0.2 \\
\hline & $\mathrm{Pu}$ & 0.4 & 0.1 & 0.3 \\
\hline & $\mathrm{Am}$ & 0.2 & 0.2 & 0.1 \\
\hline \multirow[t]{14}{*}{ Alkaline Supernate } & $\mathrm{Ce}$ & 2.1 & 4.7 & 7.4 \\
\hline & $\mathrm{Cs}$ & 17 & 80 & 255 \\
\hline & $\mathrm{Sr}$ & 1.5 & 2.3 & 2.3 \\
\hline & $\mathrm{Tc}$ & 0.6 & 0.7 & 0.6 \\
\hline & $Y$ & 2.0 & 4.3 & 5.8 \\
\hline & $\mathrm{Cr}$ & 0.1 & 0.9 & 1.5 \\
\hline & $\mathrm{Co}$ & 1.9 & 2.2 & 3.0 \\
\hline & $\mathrm{Fe}$ & $<0.1$ & $<0.1$ & $<0.1$ \\
\hline & $\mathrm{Mn}$ & 1.2 & 2.3 & 2.0 \\
\hline & $\mathrm{Zn}$ & 1.5 & 2.6 & 3.6 \\
\hline & $\mathrm{Zr}$ & $<0.1$ & $<0.1$ & $<0.1$ \\
\hline & $\mathrm{U}$ & 1.2 & 2.7 & 5.6 \\
\hline & $\mathrm{Pu}$ & 0.4 & 0.5 & 0.4 \\
\hline & $\mathrm{Am}$ & 1.7 & 3.6 & 5.3 \\
\hline
\end{tabular}


b. Cobalt Hexacyanoferrate Composite. The cobalt hexacyanoferrate content of this composite was approximately $20 \%$.

Cesium is moderately sorbed, and several other elements are weakly sorbed from acidic solution, although the sorption kinetics are slow. Cesium is more strongly sorbed from basic solution, again with slow sorption kinetics. Several other elements are moderately or weakly sorbed from basic solution.

Table III.B.4.b. Distribution of 14 Elements onto Cobalt Hexacyanoferrate Composite Resin from Two Simulant Solutions for Hanford HLW Tank 102-SY

\begin{tabular}{|c|c|c|c|c|}
\hline Medium & Absorber & 30-min Kd & 2-h Kd & 6-h Kd \\
\hline \multirow[t]{14}{*}{ Acid-Dissolved Sludge } & $\mathrm{Ce}$ & 0.6 & 1.2 & 1.0 \\
\hline & Cs & 1.9 & 5.8 & 10 \\
\hline & $\mathrm{Sr}$ & 0.9 & 1.7 & $i .5$ \\
\hline & $\mathrm{Tc}$ & 0.6 & 1.4 & 1.3 \\
\hline & $\mathrm{Y}$ & 0.8 & 1.2 & 1.2 \\
\hline & $\mathrm{Cr}$ & 0.8 & 2.0 & 1.4 \\
\hline & Co & 0.8 & 1.5 & 1.3 \\
\hline & $\mathrm{Fe}$ & 0.1 & 0.1 & $<0.1$ \\
\hline & $\mathrm{Mn}$ & $<0.1$ & 0.3 & 0.5 \\
\hline & $\mathrm{Zn}$ & 0.2 & 0.3 & 0.5 \\
\hline & $\mathrm{Zr}$ & 1.1 & 1.6 & 1.9 \\
\hline & $\mathrm{U}$ & 0.4 & 0.1 & 0.6 \\
\hline & $\mathrm{Pu}$ & 0.2 & 0.3 & 0.6 \\
\hline & $\mathrm{Am}$ & $<0.1$ & $<0.1$ & 0.1 \\
\hline \multirow[t]{14}{*}{ Alkaline Supernate } & $\mathrm{Ce}$ & 1.3 & 2.8 & 3.9 \\
\hline & Cs & 39 & 263 & 880 \\
\hline & $\mathrm{Sr}$ & 0.5 & 1.2 & 1.4 \\
\hline & $\mathrm{Tc}$ & 0.6 & 0.4 & 0.6 \\
\hline & $\mathrm{Y}$ & 1.7 & 3.5 & 4.0 \\
\hline & $\mathrm{Cr}$ & $<0.1$ & 0.5 & 0.7 \\
\hline & Co & 1.8 & 3.4 & 5.7 \\
\hline & $\mathrm{Fe}$ & $<0.1$ & $<0.1$ & $<0.1$ \\
\hline & $\mathrm{Mn}$ & 0.9 & 1.3 & 1.4 \\
\hline & $\mathrm{Zn}$ & 1.5 & 4.1 & 8.9 \\
\hline & $\mathrm{Zr}$ & 0.2 & $<0.1$ & $<0.1$ \\
\hline & $\mathrm{U}$ & 1.2 & 2.6 & 4.0 \\
\hline & $\mathrm{Pu}$ & 0.2 & 0.1 & 0.3 \\
\hline & $\mathrm{Am}$ & 1.6 & 2.3 & 3.0 \\
\hline
\end{tabular}


5. LANL-Prepared Composites. Metal phosphate composites were prepared by loading the selected metal ion on Duolite ${ }^{\mathrm{TM}} \mathrm{C}-467$ phosphonate cation exchange resin, obtained from Rohm \& Haas Co., Philadelphia, PA, and then heating the loaded resin stepwise in a tube furnace (in a stream of nitrogen) to a final temperature of $600^{\circ} \mathrm{C}$. We first tested this technique by heating $1.002 \mathrm{~g}$ of unloaded, air-dried Duolite ${ }^{\mathrm{TM}}$ resin for $2 \mathrm{~h}$ at $400^{\circ} \mathrm{C}$. and found that the resin weight had decreased to $589 \mathrm{mg}$. After the same portion of resin was heated for an additional hour at $500^{\circ} \mathrm{C}$ and one more hour at $850^{\circ} \mathrm{C}$, only $453 \mathrm{mg}$ remained.

a. Zirconium Phosphate. An 8.75-g portion of solid $\mathrm{ZrCl}_{4}$ was dissolved (with quite a vigorous reaction) in $50 \mathrm{ml}$ of $1.5 \mathrm{M} \mathrm{HCl}$. This solution was added to $50 \mathrm{ml}$ of wet Duolite ${ }^{\mathrm{TM}} \mathrm{C}$ 467 resin and mixed on an extraction wheel for $2 \mathrm{~h}$. The zirconium-loaded resin then was transferred to a column, washed with an excess of $0.5 \mathrm{M} \mathrm{HCl}$, rinsed well with water, and air-dried.

After stepwise heating to $600^{\circ} \mathrm{C}$, the weight of an initial $3.126 \mathrm{~g}$ of zirconium-loaded, airdried resin decreased to $1.177 \mathrm{~g}(37.7 \%)$. Stepwise heating of a separate 7.714 -g portion resulted in a weight of $3.772 \mathrm{~g}(48.9 \%)$. This indicates that larger portions are less efficiently decomposed to the metal phosphates. The two portions were combined for this study.

None of the 14 elements studied sorbs at useful levels from acidic or basic solution. 
Table III.B.5.a. Distribution of 14 Elements onto Zirconium Phosphate Composite from Two Simulant Solutions for Hanford HLW Tank 102-SY

\begin{tabular}{|c|c|c|c|c|}
\hline Medium & Absorber & 30-min Kd & 2-h Kd & 6-h Kd \\
\hline \multirow[t]{14}{*}{ Acid-Dissolved Sludge } & $\mathrm{Ce}$ & 0.9 & 0.4 & 0.6 \\
\hline & Cs & 1.0 & 1.1 & 1.4 \\
\hline & $\mathrm{Sr}$ & 1.0 & 1.0 & 1.2 \\
\hline & $\mathrm{Tc}$ & 1.0 & 1.0 & 1.2 \\
\hline & $\mathrm{Y}$ & 1.1 & 0.3 & 0.8 \\
\hline & $\mathrm{Cr}$ & 1.4 & 1.1 & 1.3 \\
\hline & $\mathrm{Co}$ & 1.3 & 1.3 & 1.1 \\
\hline & $\mathrm{Fe}$ & 0.3 & 0.1 & 0.3 \\
\hline & $\mathrm{Mn}$ & $<0.1$ & $<0.1$ & 0.2 \\
\hline & $\mathrm{Zn}$ & 0.2 & 0.3 & 0.2 \\
\hline & $\mathrm{Zr}$ & 2.0 & 2.4 & 3.3 \\
\hline & $\mathrm{U}$ & 0.2 & 0.1 & 0.5 \\
\hline & $\mathrm{Pu}$ & 0.5 & 0.3 & 0.6 \\
\hline & $\mathrm{Am}$ & $<0.1$ & 0.2 & 0.1 \\
\hline \multirow[t]{14}{*}{ Alkaline Supernate } & $\mathrm{Ce}$ & 0.2 & 0.8 & 0.8 \\
\hline & $\mathrm{Cs}$ & 1.3 & 2.2 & 2.6 \\
\hline & $\mathrm{Sr}$ & $<0.1$ & 0.2 & 0.1 \\
\hline & $\mathrm{Tc}$ & 0.7 & 1.0 & 0.6 \\
\hline & Y & 1.0 & 1.2 & 1.0 \\
\hline & $\mathrm{Cr}$ & 0.3 & 0.3 & 0.4 \\
\hline & Co & 0.7 & 0.6 & 0.4 \\
\hline & $\mathrm{Fe}$ & $<0.1$ & $<0.1$ & $<0.1$ \\
\hline & $\mathrm{Mn}$ & 0.3 & 0.6 & 0.5 \\
\hline & $\mathrm{Zn}$ & 0.4 & 0.7 & 0.9 \\
\hline & $\mathrm{Zr}$ & 0.7 & 0.2 & 0.2 \\
\hline & $U$ & 1.4 & 2.5 & 2.6 \\
\hline & $\mathrm{Pu}$ & $<0.1$ & 0.1 & 0.3 \\
\hline & $\mathrm{Am}$ & 0.3 & 0.5 & 0.4 \\
\hline
\end{tabular}


b. Titanium Phosphate. A 7.10-g portion of liquid $\mathrm{TiCl}_{4}$ was mixed (with quite a vigorous reaction) in $50 \mathrm{ml}$ of $1.5 \mathrm{M} \mathrm{HCl}$. This solution was added to $50 \mathrm{ml}$ of wet resin and mixed on an extraction wheel for $2 \mathrm{~h}$. The titanium-loaded resin then was transferred to a column, washed with an excess of $0.5 \mathrm{M} \mathrm{HCl}$, rinsed well with water, and air-dried.

After stepwise heating to $600^{\circ} \mathrm{C}$, the weight of an initial $5.610 \mathrm{~g}$ of this titanium-loaded, airdried resin decreased to $2.409 \mathrm{~g}(42.9 \%)$. Stepwise heating of a separate $5.669 \mathrm{~g}$-portion resulted in a weight of $2.443 \mathrm{~g}(13.1 \%)$. This indicates that when the weights of the initial portions are reasonably constant, the decomposition to metal phosphates is fairly reproducible. The two portions were combined for this study.

None of the 14 elements sorbs at useful levels from acidic or basic solution.

Table III.B.5.b. Distribution of 14 Elements onto Titanium Phosphate Composite from Two Simulant Solutions for Hạford HLW Tank 102-SY

\begin{tabular}{|c|c|c|c|c|}
\hline Medium & Absorber & 30-min Kd & 2-h Kd & 6-h Kd \\
\hline \multirow[t]{14}{*}{ Acid-Dissolved Sludge } & $\mathrm{Ce}$ & 0.6 & 0.3 & 0.3 \\
\hline & $\mathrm{Cs}$ & 2.0 & 2.4 & 4.0 \\
\hline & $\mathrm{Sr}$ & 1.0 & 0.4 & 0.7 \\
\hline & $\mathrm{Tc}$ & 1.6 & 0.7 & 0.9 \\
\hline & $\mathrm{Y}$ & 1.0 & 0.5 & 0.4 \\
\hline & $\mathrm{Cr}$ & 1.2 & 1.1 & 0.8 \\
\hline & $\mathrm{Co}$ & 0.7 & 0.2 & 0.5 \\
\hline & $\mathrm{Fe}$ & 0.1 & 0.1 & 0.1 \\
\hline & $\mathrm{Mn}$ & $<0.1$ & $<0.1$ & 0.1 \\
\hline & $\mathrm{Zn}$ & 0.2 & 0.2 & 0.2 \\
\hline & $\mathrm{Zr}$ & 1.8 & 1.7 & 2.1 \\
\hline & $U$ & 0.4 & 0.3 & 0.3 \\
\hline & $\mathrm{Pu}$ & 0.6 & 0.7 & 0.8 \\
\hline & Am & $<0.1$ & $<0.1$ & $<0.1$ \\
\hline \multirow[t]{14}{*}{ Alkaline Supernate } & $\mathrm{Ce}$ & 1.0 & 0.8 & 0.9 \\
\hline & Cs & 2.1 & 2.6 & 3.5 \\
\hline & $\mathrm{Sr}$ & 0.4 & 0.1 & 0.1 \\
\hline & $\mathrm{Tc}$ & 1.1 & 0.7 & 0.7 \\
\hline & $\mathrm{Y}$ & 1.2 & 0.9 & 0.8 \\
\hline & $\mathrm{Cr}$ & 0.3 & 0.5 & 0.3 \\
\hline & Co & 1.0 & 0.5 & 0.4 \\
\hline & $\mathrm{Fe}$ & $<0.1$ & 0.3 & 0.2 \\
\hline & $\mathrm{Mn}$ & 0.3 & 0.1 & 0.5 \\
\hline & $\mathrm{Zn}$ & 1.1 & 1.7 & 2.6 \\
\hline & $\mathrm{Zr}$ & $<0.1$ & 0.1 & 0.1 \\
\hline & $U$ & 2.5 & 3.6 & 3.8 \\
\hline & $\mathrm{Pu}$ & $<0.1$ & 0.5 & 0.2 \\
\hline & $\mathrm{Am}$ & 1.1 & 1.0 & 0.9 \\
\hline
\end{tabular}

Metal oxide composites were prepared by sorbing a dilution of the appropriate metal alkoxide into the pores of Ambersorb ${ }^{\mathrm{TM}} 563$ porous carbon beads and allowing the volatile diluent to evaporate. The loaded Ambersorb ${ }^{\mathrm{TM}}$ beads then were heated in dilute acid to hydrolyze the metal alkoxide to the corresponding metal oxide, ${ }^{14}$ after which the beads were air-dried to constant weight. 
c. Titanium Dioxide. A $30-\mathrm{ml}$ portion of titanium butoxide was diluted with $60 \mathrm{ml}$ of cyclohexane. Then 79.1 g of Ambersorb ${ }^{\mathrm{TM}} 563$ porous carbon beads, obtained from Rohm \& Halas, Philadelphia, PA, was added to absorb the liquid. After the volatile solvent evaporated, the beads were heated in $0.1 \mathrm{M}$ nitric acid for $2 \mathrm{~h}$. The loaded beads were washed with an excess of water and air-dried to yield $94.3 \mathrm{~g}$ (119.3\% of the added Ambersorb ${ }^{\mathrm{TM}}$ beads). Assuming the $\mathrm{TiO}_{2}$ was anhydrous, which it is not, this would represent 2.4 mmoles of Ti per initial g of Ambersorb ${ }^{i \mathrm{M}}$ beads.

Technetium and zirconium sorb from acidic solution, although the sorption kinetics are slow. Technetium is more strongly sorbed and uranium is moderately sorbed from basic solution.

Table III.B.5.c. Distribution of 14 Elements onto Titanium Dioxide Composite from Two Simulant Solutions for Hanford HLW Tank 102-SY

\begin{tabular}{|c|c|c|c|c|}
\hline Medium & Absorber & 30-min Kd & 2-h Kd & 6-h Kd \\
\hline \multirow[t]{14}{*}{ Acid-Dissolved Sludge } & $\mathrm{Ce}$ & 0.8 & 0.8 & 0.6 \\
\hline & $\mathrm{Cs}$ & 0.8 & 0.4 & 0.3 \\
\hline & $\mathrm{Sr}$ & 0.3 & 0.1 & 0.1 \\
\hline & $\mathrm{Tc}$ & 19 & 40 & 57 \\
\hline & $\mathrm{Y}$ & 0.8 & 0.8 & 0.6 \\
\hline & $\mathrm{Cr}$ & 0.5 & 0.6 & 0.5 \\
\hline & Co & 0.6 & 0.7 & 0.6 \\
\hline & $\mathrm{Fe}$ & $<0.1$ & 0.1 & 0.1 \\
\hline & $\mathrm{Mn}$ & 0.3 & 0.1 & 0.1 \\
\hline & $\mathrm{Zn}$ & 0.4 & 0.2 & 0.2 \\
\hline & $\mathrm{Zr}$ & 4.4 & 10 & 28 \\
\hline & $\mathrm{U}$ & 0.3 & 0.2 & 0.4 \\
\hline & $\mathrm{Pu}$ & 1.1 & 1.6 & 2.1 \\
\hline & Am & 0.2 & 0.2 & 0.2 \\
\hline \multirow[t]{14}{*}{ Alkaline Supernate } & $\mathrm{Ce}$ & 1.7 & 2.2 & 1.6 \\
\hline & $\mathrm{Cs}$ & 0.1 & $<0.1$ & $<0.1$ \\
\hline & $\mathrm{Sr}$ & $<0.1$ & 0.1 & 0.1 \\
\hline & $\mathrm{Tc}$ & 54 & 185 & 290 \\
\hline & $\mathrm{Y}$ & 0.7 & 0.8 & 0.5 \\
\hline & $\mathrm{Cr}$ & 0.4 & 1.1 & 1.7 \\
\hline & Co & 0.4 & 0.7 & 0.4 \\
\hline & $\mathrm{Fe}$ & $<0.1$ & 0.5 & 1.0 \\
\hline & $\mathrm{Mn}$ & 0.4 & 0.1 & $0 . \mathrm{i}$ \\
\hline & $\mathrm{Zn}$ & $<0.1$ & $<0.1$ & 0.2 \\
\hline & $\mathrm{Zr}$ & 0.4 & 0.8 & 1.0 \\
\hline & $\mathrm{U}$ & 3.8 & 9.1 & 19 \\
\hline & $\mathrm{Pu}$ & 0.3 & 0.5 & 1.1 \\
\hline & Am & 1.3 & 1.5 & 1.5 \\
\hline
\end{tabular}


d. Zirconium Dioxide. A 30-ml portion of zirconium butoxide.butanol was diluted with $60 \mathrm{ml}$ of cyclohexane. Then $80.4 \mathrm{~g}$ of Ambersorb ${ }^{\mathrm{MN}} 563$ porous carbon beads, obtained lrom Rohm \& Haas, Philadelphia, PA, was added to absorb the liquid. After the volatile solvent evapo)rated, the beads were heated in $0.1 \mathrm{M}$ nitric acid for $2 \mathrm{~h}$. The loaded beads were washed with an excess of water and then air-dried to yield $91.33 \mathrm{~g}$ (123.2\% of the added Ambersorb ${ }^{\mathrm{TM}}$ buads). Assuming the $\mathrm{ZrO}_{2}$ was anhydrous, which it is not, this would represent 1.1 mmoles of $\mathrm{Zr}$ per initial $g$ of Ambersorb ${ }^{\mathrm{TM}}$ beads.

Technetium is well sorbed and zirconium is weakly sorbed from acidic solution, boti: with slow sorption kinetics. Technetium is more strongly and selectively sorbed from basic solution, again with slow sorption kinetics.

Table III.B.5.d. Distribution of 14 Elements onto Zirconium Dioxide Composite from Two Simulant Solutions for Hanford HLW Tank 102-SY

\begin{tabular}{|c|c|c|c|c|}
\hline Medium & Absorber & 30-min Kd & 2-h Kd & 6-h Kd \\
\hline \multirow[t]{14}{*}{ Acid-Dissolved Sludge } & $\mathrm{Ce}$ & 0.7 & 0.8 & 0.8 \\
\hline & $\mathrm{Cs}$ & 0.5 & 0.3 & 0.5 \\
\hline & $\mathrm{Sr}$ & 0.9 & 0.2 & 0.3 \\
\hline & $\mathrm{Tc}$ & 17 & 41 & 60 \\
\hline & $\mathrm{Y}$ & 0.8 & 1.0 & 1.0 \\
\hline & $\mathrm{Cr}$ & 1.0 & 0.7 & 0.6 \\
\hline & Co & 0.8 & 0.5 & 0.5 \\
\hline & $\mathrm{Fe}$ & 0.6 & 0.2 & 0.2 \\
\hline & $\mathrm{Mn}$ & $<0.1$ & 0.2 & 0.1 \\
\hline & $\mathrm{Zn}$ & 0.2 & 0.2 & 0.2 \\
\hline & $\mathrm{Zr}$ & 1.7 & 2.8 & 3.7 \\
\hline & $\mathrm{U}$ & 0.1 & $<0.1$ & 0.3 \\
\hline & $\mathrm{Pu}$ & 0.6 & 0.7 & 0.8 \\
\hline & $\mathrm{Am}$ & 0.4 & 0.2 & 0.3 \\
\hline \multirow[t]{14}{*}{ Alkaline Supernate } & $\mathrm{Ce}$ & $<0.1$ & 0.2 & 0.6 \\
\hline & $\mathrm{Cs}$ & $<0.1$ & $<0.1$ & 0.1 \\
\hline & $\mathrm{Sr}$ & 1.0 & 0.6 & 0.6 \\
\hline & Tc & 35 & 148 & 258 \\
\hline & $\mathrm{Y}$ & 0.1 & $<0.1$ & $<0.1$ \\
\hline & $\mathrm{Cr}$ & 0.4 & 0.6 & 1.2 \\
\hline & Co & 0.4 & 0.6 & 0.5 \\
\hline & $\mathrm{Fe}$ & $<0.1$ & 0.6 & 0.4 \\
\hline & $\mathrm{Mn}$ & 0.1 & 0.7 & 0.5 \\
\hline & $\mathrm{Zn}$ & 0.3 & 0.3 & 0.3 \\
\hline & $\mathrm{Zr}$ & $<0.1$ & 0.7 & 0.9 \\
\hline & $\mathrm{U}$ & 1.4 & 2.2 & 2.9 \\
\hline & $\mathrm{Pu}$ & 0.1 & 0.4 & 0.6 \\
\hline & $\mathrm{Am}$ & $<0.1$ & $<0.1$ & $<0.1$ \\
\hline
\end{tabular}


e. Niobium Pentoxide. A 50-ml portion of niobium ethoxide was diluted with $100 \mathrm{ml}$ of cyclohexane. Then $113.9 \mathrm{~g}$ of Ambersorb ${ }^{\mathrm{TM}} 56.3$ porous carbon beads, obtained from Rohm \& Hats, Philadelphia, PA, was added to absorb the liquid. After the volatile solvent evaporated, the beads were heated in $0.1 \mathrm{M}$ nitric acid for $2 \mathrm{~h}$. The loaded beads were washed with an excess of water and air-dried to yield $140.30 \mathrm{~g}\left(123.2 \%\right.$ of the added Ambersorb ${ }^{1 \mathrm{~N}}$ beads). Assuming the $\mathrm{Nb}_{2} \mathrm{O}_{5}$ was anhydrous, which it is not, this would represent 1.7 mmoles of $\mathrm{Nb}$ per initial g of Ambersorb ${ }^{\mathrm{TM}}$ beads.

Technetium and zirconium sorb moderately from acidic solution. Technetium is more strongly sorbed from basic solution, although with slow sorption kinetics. Several other elements sorb moderately or weakly from basic solution.

Table III.B.5.e. Distribution of 14 Elements onto Niobium Pentoxide Composite from Two Simulant Solutions for Hanford HLW Tank 102-SY

\begin{tabular}{|c|c|c|c|c|}
\hline Medium & Absorber & 30-min Kd & 2-h Kd & 6-h Kd \\
\hline \multirow[t]{14}{*}{ Acid-Dissolved Sludge } & $\mathrm{Ce}$ & 0.3 & 0.5 & 0.5 \\
\hline & $\mathrm{Cs}$ & 0.5 & 0.9 & 0.7 \\
\hline & $\mathrm{Sr}$ & 0.1 & 0.3 & 0.1 \\
\hline & $\mathrm{Tc}$ & 6.9 & 17 & 31 \\
\hline & $\mathrm{Y}$ & 0.8 & 0.3 & 0.5 \\
\hline & $\mathrm{Cr}$ & 0.6 & 0.5 & 0.6 \\
\hline & $\mathrm{Co}$ & 0.3 & 0.4 & 0.6 \\
\hline & $\mathrm{Fe}$ & 0.5 & 0.3 & 0.4 \\
\hline & $\mathrm{Mn}$ & 0.1 & 0.2 & 0.1 \\
\hline & $\mathrm{Zn}$ & 0.3 & 0.3 & 0.3 \\
\hline & $\mathrm{Zr}$ & 2.2 & 6.3 & 14 \\
\hline & $\mathrm{U}$ & $<0.1$ & 0.6 & 0.5 \\
\hline & $\mathrm{Pu}$ & 1.5 & 2.4 & 3.4 \\
\hline & $\mathrm{Am}$ & 0.2 & 0.1 & 0.1 \\
\hline \multirow[t]{14}{*}{ Alkaline Supernate } & $\mathrm{Ce}$ & 1.7 & 2.4 & 2.8 \\
\hline & $\mathrm{Cs}$ & 4.5 & 5.6 & 6.8 \\
\hline & $\mathrm{Sr}$ & 0.5 & 0.1 & 0.2 \\
\hline & $\mathrm{Tc}$ & 28 & 1.34 & 271 \\
\hline & $\mathrm{Y}$ & 0.9 & 0.8 & 0.8 \\
\hline & $\mathrm{Cr}$ & 0.9 & 1.6 & 2.9 \\
\hline & Co & 0.4 & 0.6 & 0.6 \\
\hline & $\mathrm{Fe}$ & $<0.1$ & $<0.1$ & 0.2 \\
\hline & $\mathrm{Mn}$ & 0.8 & 0.7 & 0.3 \\
\hline & $\mathrm{Zn}$ & 1.3 & 0.8 & 0.7 \\
\hline & $\mathrm{Zr}$ & $<0.1$ & 0.2 & 0.6 \\
\hline & $\mathrm{U}$ & 2.3 & 4.1 & 7.3 \\
\hline & $\mathrm{Pu}$ & 0.5 & 0.2 & 0.2 \\
\hline & $\mathrm{Am}$ & 1.1 & 1.7 & 2.9 \\
\hline
\end{tabular}


6. Liquid Extractants. We prepared and evaluated porous beads impregnilted with 12 different liquid extractants.

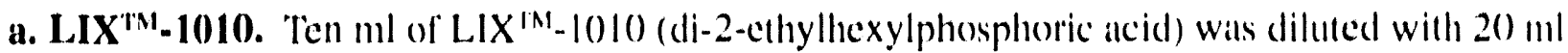
of cyclohexane, after which $25.76 \mathrm{~g}$ of Ambersorb ${ }^{\mathrm{N}} 56.3$ porous carbon beads, obtained from Rohm \& Haas Co.. Philadelphia, PA, was added to absorb the liquid. The cyclohexance evalporalled to yield $34.7 \mathrm{~g}$ of the loaded Ambersorb ${ }^{\text {IMI }}$ beads.

Zirconium is moderately sorbed and technetium is weakly sorbed firom acidic solution, both with slow sorption kinetics. From basic solution, americium sorption is significantly greater than lanthanides sorption, which demonstrates a potential for an efficient trivalent actinide/lanthanide group separation. Uranium, zinc, and manganese are moderately so: ned from basic solution.

Table III.B.6.a. Distribution of 14 Elements into LIX ${ }^{T M}-1010$ trom Two Simulant Solutions for Hanford HLW Tank 102-SY

\begin{tabular}{|c|c|c|c|c|}
\hline Medium & Absorber & 30-min Kd & 2-h Kd & 6-h Kd \\
\hline \multirow[t]{14}{*}{ Acid-Dissolved Sludge } & $\mathrm{Ce}$ & 0.8 & 0.9 & 0.6 \\
\hline & Cs & $<0.1$ & 0.3 & 0.1 \\
\hline & $\mathrm{Sr}$ & $<0.1$ & 0.2 & 0.1 \\
\hline & $\mathrm{Tc}$ & 1.8 & 2.7 & 4.0 \\
\hline & $\mathrm{Y}$ & 1.1 & 0.5 & 0.4 \\
\hline & $\mathrm{Cr}$ & 0.8 & 0.8 & 0.7 \\
\hline & Co & 0.4 & 0.1 & 0.2 \\
\hline & $\mathrm{Fe}$ & 0.4 & 0.3 & 0.5 \\
\hline & $\mathrm{Mn}$ & $<0.1$ & 0.2 & 0.1 \\
\hline & $\mathrm{Zn}$ & $<0.1$ & 0.4 & 0.2 \\
\hline & $\mathrm{Zr}$ & 3.6 & 10 & 25 \\
\hline & $\mathrm{U}$ & 0.1 & $<0.1$ & 0.5 \\
\hline & $\mathrm{Pu}$ & 0.8 & 0.7 & 0.9 \\
\hline & Am & 0.1 & 0.1 & 0.1 \\
\hline \multirow[t]{14}{*}{ Alkaline Supernate } & $\mathrm{Ce}$ & 0.5 & 1.5 & 1.7 \\
\hline & $\mathrm{Cs}$ & $<0.1$ & $<0.1$ & 0.2 \\
\hline & $\mathrm{Sr}$ & 0.7 & 0.7 & 0.4 \\
\hline & $\mathrm{Tc}$ & 1.0 & 1.7 & 3.0 \\
\hline & $\mathrm{Y}$ & 1.0 & 1.2 & 1.3 \\
\hline & $\mathrm{Cr}$ & 0.3 & 0.6 & 0.6 \\
\hline & $\mathrm{Co}$ & 1.0 & 0.9 & 0.6 \\
\hline & $\mathrm{Fe}$ & $<0.1$ & 0.1 & $<0.1$ \\
\hline & $\mathrm{Mn}$ & 4.9 & 5.7 & 5.3 \\
\hline & $\mathrm{Zn}$ & 3.6 & 4.0 & 4.0 \\
\hline & $\mathrm{Zr}$ & 0.1 & 0.8 & 0.5 \\
\hline & $\mathrm{U}$ & 4.3 & 7.1 & 1.5 \\
\hline & $\mathrm{Pu}$ & 0.1 & $<0.1$ & 0.1 \\
\hline & $\mathrm{Am}$ & 10 & 27 & 64 \\
\hline
\end{tabular}


b. LIX'N-54. Ten ml of LIX'N-54 (a beta diketone) wits diluted with 20 ) ml of cyclohexinte, after which 26.80 g of Ambersorb ${ }^{1 N} 56.3$ porous curbon beads, obtained from Rohmen \& Hatus Co., Philadelphia. PA, wass added to absorb the liguid. "The cyclohexime evaporated to yield $34.6 \mathrm{~g}$ of the loaded Ambersorb ${ }^{\text {IN }}$ beids.

Zirconium is weakly sorbed from acidic solution. From basic solution, the sorption of annericiun is significenntly greater than that of lanthanides, which demonstrates a potential for an eflicient trivalent actinide/lanthanide group separation. Uranium, iron, manganese, plutonium. and technetium are weakly to moderately sorbed from basic solution.

Tuble III.B.6.b. Distribution of 14 Elements into LIX ${ }^{1 N_{-}} .54$ from Two Simulant Solutions for Hanford HLW Tank 1()2-SY

\begin{tabular}{|c|c|c|c|c|}
\hline Medium & Absorber & 3()$-\mathrm{min} \mathrm{Kd}$ & $2 \cdot \mathrm{h} \mathrm{Kd}$ & 6-h Kd \\
\hline \multirow[t]{14}{*}{ Acid-Dissolved Sludge } & $\mathrm{Ce}$ & 0.7 & 0.8 & 0.8 \\
\hline & $\mathrm{Cs}$ & 0.5 & 0.5 & 0.3 \\
\hline & $\mathrm{Sr}$ & 0.5 & 0.3 & 0.2 \\
\hline & $\mathrm{Tc}$ & 0.5 & 0.5 & 0.5 \\
\hline & $Y$ & 0.7 & 0.6 & 0.6 \\
\hline & $\mathrm{Cr}$ & 0.8 & 1.0 & 0.8 \\
\hline & $\mathrm{Co}$ & 0.9 & 0.4 & 0.5 \\
\hline & $\mathrm{Fe}$ & 0.7 & 0.3 & 0.4 \\
\hline & $\mathrm{Mn}$ & 0.3 & 0.2 & 0.2 \\
\hline & $\mathrm{Zn}$ & 0.5 & 0.3 & 0.2 \\
\hline & $\mathrm{Zr}$ & 1.4 & 1.7 & 3.4 \\
\hline & $\mathrm{U}$ & $<0.1$ & 0.1 & 0.1 \\
\hline & $\mathrm{Pu}$ & 0.5 & 0.1 & 0.1 \\
\hline & $\mathrm{Am}$ & 0.3 & 0.2 & 0.1 \\
\hline \multirow[t]{14}{*}{ Alkaline Supernate } & $\mathrm{Ce}$ & $<0.1$ & 0.4 & 0.4 \\
\hline & $\mathrm{Cs}$ & $<0.1$ & 0.3 & 0.1 \\
\hline & $\mathrm{Sr}$ & 0.8 & 0.3 & 0.6 \\
\hline & $\mathrm{Tc}$ & 1.4 & 2.8 & 4.1 \\
\hline & $\mathrm{Y}$ & 0.4 & 0.1 & 0.3 \\
\hline & $\mathrm{Cr}$ & 0.5 & 0.3 & 0.7 \\
\hline & Co & 0.5 & 1.0 & 0.9 \\
\hline & $\mathrm{Fe}$ & 2.9 & 3.4 & 4.7 \\
\hline & $\mathrm{Mn}$ & 2.4 & 2.1 & 2.6 \\
\hline & $\mathrm{Zn}$ & 0.4 & 0.3 & 0.6 \\
\hline & $\mathrm{Zr}$ & 0.1 & 0.3 & 0.4 \\
\hline & $\mathrm{U}$ & 11 & 12 & 12 \\
\hline & $\mathrm{Pu}$ & 2.2 & 2.8 & 3.0 \\
\hline & $\mathrm{Am}$ & 34 & 32 & 30 \\
\hline
\end{tabular}




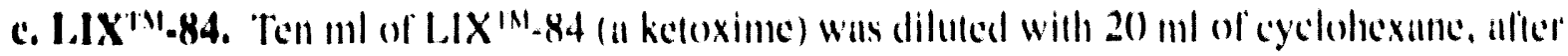
which $20.1 .3 \mathrm{~g}$ of Ambersorb ${ }^{1 \mathrm{~N}} 50.3$ porous carbon beads, obtained from Rohm \& Haus ('o.. Philadelphia, PA, was added to absorb the licpuid. "The cyclohexane evaporated a yicld 34.8 g of the louded Ambersorb ${ }^{\text {IN }}$ beids.

None of the 14 elements was significantly sorbed from acidic or basic solution.

Table III.B.6.c. Distribution of 14 Elements into LIX TM. 84 from Two Simulant Solulions for Hunford HLW Tank 102-SY

\begin{tabular}{|c|c|c|c|c|}
\hline Medium & Alssorber & $3(0-\mathrm{min} \mathrm{Kd}$ & $2 \cdot h \mathrm{Kd}$ & 6.h Kd \\
\hline \multirow[t]{14}{*}{ Acid-Dissolved Sludge } & $\mathrm{Ce}$ & 0.7 & 0.7 & 0.9 \\
\hline & $\mathrm{Cs}$ & 0.4 & 0.1 & 0.4 \\
\hline & $\mathrm{Sr}$ & $<0.1$ & 0.3 & 0.1 \\
\hline & $\mathrm{Tc}$ & 0.7 & 1.2 & 1.7 \\
\hline & $Y$ & 0.7 & 0.6 & 0.8 \\
\hline & $\mathrm{Cr}$ & 0.4 & 0.7 & 0.8 \\
\hline & $\mathrm{Co}$ & 0.6 & 1.0 & 0.7 \\
\hline & $\mathrm{Fe}$ & 1.0 & 0.5 & 0.8 \\
\hline & $\mathrm{Mn}$ & 0.9 & 0.3 & 0.5 \\
\hline & $\mathrm{Zn}$ & 0.6 & 0.2 & 0.3 \\
\hline & $\mathrm{Zr}$ & 0.8 & 1.4 & 1.8 \\
\hline & $U$ & $<0.1$ & 0.3 & 0.5 \\
\hline & $\mathrm{Pu}$ & 0.6 & 0.4 & 0.3 \\
\hline & Am & 0.7 & 0.2 & 0.2 \\
\hline \multirow[t]{14}{*}{ Alkaline Supernate } & $\mathrm{Ce}$ & 0.9 & 0.4 & 0.5 \\
\hline & $\mathrm{Cs}$ & $<0.1$ & 0.4 & 0.2 \\
\hline & $\mathrm{Sr}$ & 0.5 & 0.4 & 0.6 \\
\hline & $\mathrm{Tc}$ & 0.2 & 0.7 & 0.7 \\
\hline & $\mathrm{Y}$ & 0.2 & 0.4 & 0.4 \\
\hline & $\mathrm{Cr}$ & 1.0 & 1.3 & $1 . .3$ \\
\hline & Co & 0.8 & 0.8 & 1.1 \\
\hline & $\mathrm{Fe}$ & 0.3 & 0.6 & 0.5 \\
\hline & $\mathrm{Mn}$ & 0.9 & 0.9 & 0.6 \\
\hline & $\mathrm{Zn}$ & 0.6 & 0.4 & 0.2 \\
\hline & $\mathrm{Zr}$ & $<0.1$ & $<0.1$ & 0.4 \\
\hline & $\mathrm{U}$ & 0.8 & 0.8 & 0.7 \\
\hline & $\mathrm{Pu}$ & 0.7 & 0.5 & 0.4 \\
\hline & $\mathrm{Am}$ & $<0.1$ & $<0.1$ & $<0.1$ \\
\hline
\end{tabular}




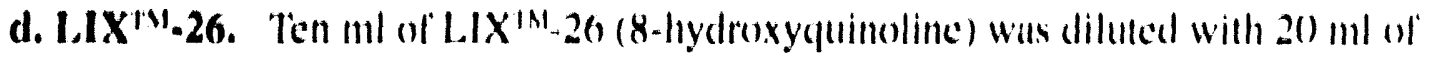

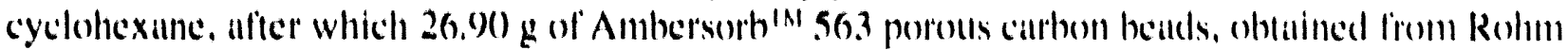

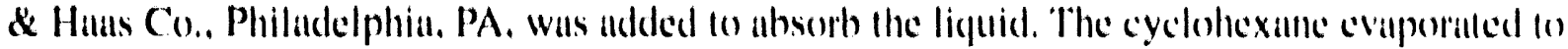
yidd .37 .1 gof the louded Ambersorb ${ }^{\text {IN }}$ heads.

Plutonium is moderately sorbed, whereats uranium and ledinetium ane weikly sorbed from acidic solution. Technetium, cerium, americium, and uranium sorb at useful levels from basic solution.

Table III.B.6.d. Distribution of 14 Elements into LIX ${ }^{1 N}-26$ from Two Simulant Sollttions for Hanford HLW Tank 102-SY

\begin{tabular}{|c|c|c|c|c|}
\hline Medlum & Absorber & 30-min Kd & $2 \cdot h \mathrm{Kd}$ & 6-h Kd \\
\hline \multirow[t]{14}{*}{ Acid-Dissolved Sludge } & $\mathrm{Ce}$ & 0.4 & 0.5 & 0.4 \\
\hline & $\mathrm{Cs}$ & 0.4 & 0.5 & 0.2 \\
\hline & $\mathrm{Sr}$ & 0.7 & 0.4 & 0.5 \\
\hline & $\mathrm{Tc}$ & 1.1 & 1.4 & 1.4 \\
\hline & $Y$ & 0.5 & 0.6 & 0.4 \\
\hline & $\mathrm{Cr}$ & 0.5 & 0.6 & 0.5 \\
\hline & $\mathrm{Co}$ & 0.4 & 0.4 & 0.5 \\
\hline & $\mathrm{Fe}$ & 0.3 & 0.6 & 0.5 \\
\hline & $\mathrm{Mn}$ & $<0.1$ & $<() .1$ & $<0.1$ \\
\hline & $\mathrm{Zn}$ & 0.5 & 0.4 & 0.3 \\
\hline & $\mathrm{Zr}$ & 0.6 & 0.6 & 0.7 \\
\hline & $U$ & 2.0 & 2.7 & 3.4 \\
\hline & $\mathrm{Pu}$ & 3.9 & 7.9 & 15 \\
\hline & $\mathrm{Am}$ & 0.4 & 0.5 & 0.4 \\
\hline \multirow[t]{14}{*}{ Alkaline Supernate } & $\mathrm{Ce}$ & 32 & 40 & 42 \\
\hline & $\mathrm{Cs}$ & $<0.1$ & $<0.1$ & $<() .1$ \\
\hline & $\mathrm{Sr}$ & 0.6 & 0.7 & 0.6 \\
\hline & $T c$ & 54 & 112 & 190 \\
\hline & $Y$ & 4.1 & 4.7 & 4.9 \\
\hline & $\mathrm{Cr}$ & $<0.1$ & 0.2 & 0.3 \\
\hline & Co & 1.3 & 1.1 & 1.1 \\
\hline & $\mathrm{Fe}$ & 0.3 & 0.3 & (). .3 \\
\hline & $\mathrm{Mn}$ & $<0.1$ & $<0.1$ & 0.1 \\
\hline & $\mathrm{Zn}$ & 0.3 & 0.2 & 0.1 \\
\hline & $\mathrm{Zr}$ & 0.8 & 0.9 & 0.7 \\
\hline & $\mathrm{U}$ & 12 & 21 & 24 \\
\hline & $\mathrm{Pu}$ & 0.2 & 0.1 & 0.2 \\
\hline & $\mathrm{Am}$ & 24 & 33 & .30 \\
\hline
\end{tabular}




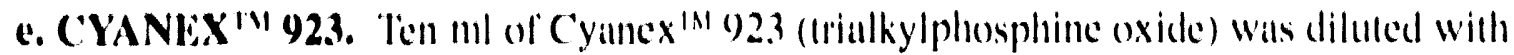

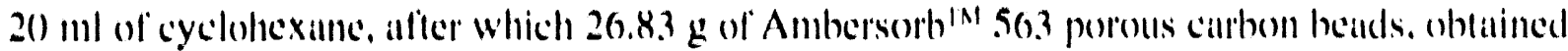
from Rohm \& Hais (o., Philadelphia, PA, was added lo absoth the liguid. The cyclohexane evaporated to yicld $36.8 \mathrm{~g}$ of the londed Ambersorb ${ }^{\text {int }}$ beads.

From acidic solution, plutonium, zirconium, uranium, and lechnedium sorb moderalely with slow sorption kinetics. Technetium, lanthanides, annericium, and uramium sorb strongly from basic solution. This extractant demonstraltes an outstanding ability to recover malny targeted elements from alkaline supernate solution.

Tuble 111.3.6.e. Distribution of 14 Elements into CYANEX ${ }^{\text {TN }} 92.3$ from Two Simulant Solutions for Hanford HLW Tank 102-SY

\begin{tabular}{|c|c|c|c|c|}
\hline Medlum & Alssorber & 3(1)-min Kd & $2 \cdot h \mathrm{Kd}$ & $6 . h \mathrm{Kd}$ \\
\hline \multirow[t]{17}{*}{ Acid-Dissulved Sludge } & $\mathrm{Cu}$ & 0.5 & 0.2 & 0.5 \\
\hline & Cs & 0.5 & 0.3 & 0.4 \\
\hline & $\mathrm{Sr}$ & 0.8 & $(0.5$ & $(0.5$ \\
\hline & $T i$ & 1.8 & 2.6 & 4.3 \\
\hline & $Y$ & 0.7 & 0.4 & $(0.7$ \\
\hline & $\mathrm{Cr}$ & $(0.8$ & 0.6 & 0.9 \\
\hline & Co & 0.5 & 0.2 & 0.3 \\
\hline & $\mathrm{Fe}$ & $<() .1$ & (1).4 & 0.6 \\
\hline & Mn & $<0.1$ & $<() .1$ & $<() .1$ \\
\hline & $\mathrm{Zn}$ & 0.2 & 0.1 & 0.2 \\
\hline & $\mathrm{Zr}$ & 2.0 & 3.1 & 7.6 \\
\hline & $U$ & $<() .1$ & 1.0 & 1.9 \\
\hline & $U^{a}$ & 2.7 & 6.5 & 14 \\
\hline & Pu & 3.0 & 7.6 & 21 \\
\hline & $P u^{4}$ & 21 & 100 & 199 \\
\hline & Am & 0.3 & $0 . .3$ & 0.3 \\
\hline & $A m^{\prime \prime}$ & 0.4 & 0.4 & 0.4 \\
\hline \multirow[t]{14}{*}{ Alkaline Supernate } & $\mathrm{Ce}$ & 744 & 987 & 1596 \\
\hline & Cs & 0.8 & 0.5 & 0.4 \\
\hline & $\mathrm{Sr}$ & ().8 & 0.5 & 0.6 \\
\hline & $\mathrm{TC}$ & $3(0)$ & 5.51 & 582 \\
\hline & $Y$ & 1078 & 2014 & 2981 \\
\hline & $\mathrm{Cr}$ & $<() .1$ & $<() .1$ & 0.3 \\
\hline & $\left.C_{0}\right)$ & 0.6 & 0.7 & 0.7 \\
\hline & $\mathrm{Fc}$ & $<() .1$ & $<(0.1$ & $<() .1$ \\
\hline & $M_{n}$ & 0.4 & 0.9 & 0.7 \\
\hline & $7 n$ & 2.9 & 4.4 & 4.3 \\
\hline & $\mathrm{Zr}$ & $0 . \%$ & 0.4 & 0.8 \\
\hline & U & 122 & 2.3 .3 & .356 \\
\hline & $\mathrm{Pu}$ & 1.4 & 1.9 & 2.0 \\
\hline & Am & 538 & 7.51 & 8() 2 \\
\hline
\end{tabular}

"These solutions contained $60 \mathrm{mg} \mathrm{Pu} / \mathrm{L}$; all others contained $3 \mathrm{~g} \mathrm{Pu} / \mathrm{L}$. 
f. CYANEX'M 272. Ten ml of Cyanex ${ }^{1 N} 272$ (trimethylpentylphosphoric acid) was diluted with $20 \mathrm{ml}$ of cyclohexane, aller which $26.49 \mathrm{~g}$ of Ambersorb $1 \mathrm{M} 56.3$ porous ciarbon beads. obtained from Rohm \& Hatas Co., Philadelphia, PA, wats added to absorth the liculid. The cyclohexane evaporated to yield $37.1 \mathrm{~g}$ of the loaded Ambersorb ${ }^{\text {IN }}$ beids.

Zirconium is strongly and selectively sorbed from acidic solution, although the sorption kinetics are slow. Uranium is selectively sorbed at a useful level from basic solution.

Table III.B.6.f. Distribution of 14 Elements into CYANE: ${ }^{\text {IN }} 272$ from Two Simulatut Solutions for Hanford HLW Tank 1(22-SY

\begin{tabular}{|c|c|c|c|c|}
\hline Medium & Absorber & 3(1)-min Kd & $2 \cdot h \mathrm{Kd}$ & 6.h Kd \\
\hline \multirow[t]{14}{*}{ Acid-Dissolved Sludge } & $\mathrm{Cc}$ & 0.4 & 0.2 & 0.5 \\
\hline & Cs & 0.3 & 0.2 & 0.3 \\
\hline & $\mathrm{Sr}$ & 0.5 & 0.2 & 0.4 \\
\hline & $\mathrm{Tc}$ & 0.4 & 0.6 & 0.7 \\
\hline & $Y$ & 0.8 & 0.7 & 0.7 \\
\hline & $\mathrm{Cr}$ & 0.5 & 0.6 & 0.8 \\
\hline & Co & 0.5 & 0.4 & 0.4 \\
\hline & $\mathrm{Fc}$ & $<0.1$ & 0.3 & 0.3 \\
\hline & $\mathrm{Mn}$ & $<0.1$ & $<0.1$ & $<() .1$ \\
\hline & $\mathrm{Zn}$ & 0.1 & 0.3 & 0.2 \\
\hline & $\mathrm{Zr}$ & 8.0 & 32 & 199 \\
\hline & $U$ & 0.3 & 1.4 & 1.4 \\
\hline & $\mathrm{Pu}$ & 0.9 & 1.3 & 2.8 \\
\hline & $\mathrm{Am}$ & $<0.1$ & 0.2 & 0.1 \\
\hline \multirow[t]{14}{*}{ Alkaline Supernate } & $\mathrm{Ce}$ & 0.1 & 0.5 & 0.2 \\
\hline & Cs & 0.4 & 0.1 & 0.1 \\
\hline & $\mathrm{Sr}$ & 0.9 & 0.6 & 0.9 \\
\hline & $\mathrm{Tc}$ & 0.5 & 0.3 & 0.5 \\
\hline & $Y$ & 0.8 & 1.0 & 1.1 \\
\hline & $\mathrm{Cr}$ & $<(0.1$ & 0.7 & 0.7 \\
\hline & Co & 0.8 & 0.7 & 0.7 \\
\hline & $\mathrm{Fe}$ & 0.5 & 0.6 & 0.6 \\
\hline & $\mathrm{Mn}$ & $<0.1$ & $<(0.1$ & $<0.1$ \\
\hline & $\mathrm{Zn}$ & 1.1 & 1.3 & 1.6 \\
\hline & $\mathrm{Zr}$ & 0.5 & 0.7 & 0.6 \\
\hline & U & 23 & 41 & 58 \\
\hline & $\mathrm{Pu}$ & 0.2 & 0.1 & 0.3 \\
\hline & $\mathrm{Am}$ & 1.2 & 1.1 & 1.3 \\
\hline
\end{tabular}


g. DHDECMP. Ten ml of DHDECMP (dihexyl-N,N-diethylcarbamoylmethylphosphonate), obtained from Occidental Chemical Corporation, Specialty Products Division, Niagara Falls, NY, was diluted with $20 \mathrm{ml}$ of cyclohexane, after which $27.99 \mathrm{~g}$ of Ambersorb ${ }^{\mathrm{TM}}$ 563 porous carbon beads, obtained from Rohm \& Haas Co., Philadelphia, PA, was add to absorb the liquid. The cyclohexane evaporated to yield $39.5 \mathrm{~g}$ of the loaded Ambersorb ${ }^{\mathrm{TM}}$ beads.

Plutonium is moderately sorbed, whereas uranium and technetium are weakly sorbed from acidic solution. From basic solution, technetium, cerium, americium, and uranium are well sorbed, whereas yttrium is weakly sorbed.

Table III.B.6.g. Distribution of 14 Elements into DHDECMP from Two Simulant Solutions for Hanford HLW Tank 102-SY

\begin{tabular}{|c|c|c|c|c|}
\hline Medium & Absorber & $30-\min \mathrm{Kd}$ & 2-h Kd & 6-h Kd \\
\hline \multirow[t]{14}{*}{ Acid-Dissolved Sludge } & $\mathrm{Ce}$ & 0.2 & 0.3 & 0.4 \\
\hline & Cs & 0.4 & 0.3 & 0.4 \\
\hline & $\mathrm{Sr}$ & 0.3 & 0.4 & 0.4 \\
\hline & $\mathrm{Tc}$ & 0.7 & 1.0 & 1.6 \\
\hline & $\mathrm{Y}$ & 0.5 & 1.0 & 0.6 \\
\hline & $\mathrm{Cr}$ & 0.8 & 0.8 & 0.7 \\
\hline & $\mathrm{Co}$ & 0.5 & 0.4 & 0.2 \\
\hline & $\mathrm{Fe}$ & 0.2 & 0.3 & 0.3 \\
\hline & $\mathrm{Mn}$ & $<0.1$ & $<0.1$ & $<0.1$ \\
\hline & $\mathrm{Zn}$ & 0.2 & 0.3 & 0.2 \\
\hline & $\mathrm{Zr}$ & 0.9 & 0.6 & 0.9 \\
\hline & $\mathrm{U}$ & 1.3 & 2.2 & 3.2 \\
\hline & $\mathrm{Pu}$ & 4.4 & 8.2 & 15 \\
\hline & $\mathrm{Am}$ & 0.4 & 0.6 & 0.4 \\
\hline \multirow[t]{14}{*}{ Alkaline Supernate } & $\mathrm{Ce}$ & 26 & 45 & 51 \\
\hline & $\mathrm{Cs}$ & $<0.1$ & $<0.1$ & $<0.1$ \\
\hline & $\mathrm{Sr}$ & 0.5 & 0.6 & 0.7 \\
\hline & $\mathrm{Tc}$ & 43 & 112 & 222 \\
\hline & $\mathrm{Y}$ & 3.4 & 5.1 & 5.8 \\
\hline & $\mathrm{Cr}$ & 0.3 & 0.2 & 0.6 \\
\hline & Co & 0.8 & 0.9 & 1.1 \\
\hline & $\mathrm{Fe}$ & $<0.1$ & $<0.1$ & $<0.1$ \\
\hline & $\mathrm{Mn}$ & 0.6 & 0.5 & 0.4 \\
\hline & $\mathrm{Zn}$ & 0.7 & 0.4 & 0.5 \\
\hline & $\mathrm{Zr}$ & $<0.1$ & 0.5 & 0.8 \\
\hline & $\mathrm{U}$ & 22 & 31 & 33 \\
\hline & $\mathrm{Pu}$ & 0.4 & 0.3 & 0.4 \\
\hline & Am & 25 & 34 & 35 \\
\hline
\end{tabular}


h. DHDECMP-DIPB. Ten $\mathrm{ml}$ of DHDECMP was diluted with $10 \mathrm{ml}$ of DIPB

(diisopropylbenzene) and $30 \mathrm{ml}$ of cyclohexane, after which $48.45 \mathrm{~g}$ of Ambersorb ${ }^{\mathrm{TM}} 563$ porous carbon beads, obtained from Rohm \& Haas Co., Philadelphia, PA, was added to absorb the liquid. The cyclohexane evaporated to yield $65.3 \mathrm{~g}$ of the loaded Ambersorb ${ }^{\mathrm{TM}}$ beads.

Plutonium is moderately sorbed from acidic solution. From basic solution, technetium, cerium, americium, and uranium are sorbed at useful levels, whereas yttrium is weakly sorbed.

Table III.B.6.h. Distribution of 14 Elements into DHDECMP-DIPB from Two Simulant Solutions for Hanford HLW Tank 102-SY

\begin{tabular}{|c|c|c|c|c|}
\hline Medium & Absorber & 30-min Kd & 2-h Kd & 6-h Kc \\
\hline \multirow[t]{14}{*}{ Acid-Dissolved Sludge } & $\mathrm{Ce}$ & $<0.1$ & 0.4 & 0.6 \\
\hline & $\mathrm{Cs}$ & 0.1 & $<0.1$ & 0.2 \\
\hline & $\mathrm{Sr}$ & 0.2 & 0.1 & 0.4 \\
\hline & $\mathrm{Tc}$ & 0.6 & 1.1 & 1.5 \\
\hline & $\mathrm{Y}$ & 0.3 & 0.4 & 0.6 \\
\hline & $\mathrm{Cr}$ & 0.5 & 0.7 & 0.9 \\
\hline & Co & 0.1 & 0.4 & 0.5 \\
\hline & $\mathrm{Fe}$ & 0.3 & 0.5 & 0.3 \\
\hline & $\mathrm{Mn}$ & $<0.1$ & $<0.1$ & $<0.1$ \\
\hline & $\mathrm{Zn}$ & 0.3 & 0.5 & 0.2 \\
\hline & $\mathrm{Zr}$ & 0.4 & 0.8 & 1.0 \\
\hline & $\mathrm{U}$ & 1.2 & 2.3 & 2.7 \\
\hline & $\mathrm{Pu}$ & 2.9 & 5.5 & 10 \\
\hline & $\mathrm{Am}$ & 0.4 & 0.6 & 0.3 \\
\hline \multirow[t]{14}{*}{ Alkaline Supernate } & $\mathrm{Ce}$ & 18 & 28 & 28 \\
\hline & Cs & 0.1 & $<0.1$ & 0.2 \\
\hline & $\mathrm{Sr}$ & 0.5 & 0.7 & 0.4 \\
\hline & $\mathrm{Tc}$ & 27 & 49 & 62 \\
\hline & $\mathrm{Y}$ & 2.9 & 3.3 & 3.1 \\
\hline & $\mathrm{Cr}$ & 0.6 & 0.5 & 0.5 \\
\hline & Co & 0.6 & 0.9 & 0.6 \\
\hline & $\mathrm{Fe}$ & 0.1 & 0.3 & 0.4 \\
\hline & $\mathrm{Mn}$ & $<0.1$ & 0.3 & 0.2 \\
\hline & $\mathrm{Zn}$ & 0.2 & 0.3 & 0.1 \\
\hline & $\mathrm{Zr}$ & 0.4 & 0.5 & 0.5 \\
\hline & $\mathrm{U}$ & 9.3 & 16 & 18 \\
\hline & $\mathrm{Pu}$ & 0.7 & 0.6 & 0.4 \\
\hline & $\mathrm{Am}$ & 14 & 21 & 19 \\
\hline
\end{tabular}


i. CMPO-DIPB. Ten ml of CMPO (octylphenyl-N,N-diisobutylcarbamoyl-methylphosphine oxide), obtained from M\&T Chemicals, Inc., Rahway, NJ, was diluted with $10 \mathrm{ml}$ of DIPB (diisopropylbenzene) and $30 \mathrm{ml}$ of cyclohexane, after which $46.83 \mathrm{~g}$ of Ambersorb ${ }^{\mathrm{TM}} 563$ porous carbon beads, obtained from Rohm \& Haas Co., Philadelphia, PA, was added to absorb the liquid. The cyclohexane evaporated to yield $64.1 \mathrm{~g}$ of the loaded Ambersor ${ }^{\mathrm{TM}}$ beads.

None of the 14 elements sorbed at useful levels from acidic solution. Technetium, cerium, americium, uranium, and yttrium are well sorbed from basic solution, although the sorption kinetics are slow.

Table III.B.6.i. Distribution of 14 Elements into CMPO-DIPB from Two Simulant Solutions for Hanford HLW Tank 102-SY

\begin{tabular}{|c|c|c|c|c|}
\hline Medium & Absorber & $30-\operatorname{min~Kd}$ & 2-h Kd & 6-h Kd \\
\hline \multirow[t]{14}{*}{ Acid-Dissolved Sludge } & $\mathrm{Ce}$ & 0.3 & 0.3 & 0.6 \\
\hline & $\mathrm{Cs}$ & 0.5 & 0.2 & 0.3 \\
\hline & $\mathrm{Sr}$ & 0.5 & 0.4 & 0.3 \\
\hline & $\mathrm{Tc}$ & 0.6 & 1.1 & 2.2 \\
\hline & $\mathrm{Y}$ & 0.5 & 0.6 & 0.5 \\
\hline & $\mathrm{Cr}$ & 0.7 & 0.8 & 0.9 \\
\hline & Co & 0.2 & 0.1 & 0.1 \\
\hline & $\mathrm{Fe}$ & 0.6 & 0.6 & 0.5 \\
\hline & $\mathrm{Mn}$ & $<0.1$ & $<0.1$ & $<0.1$ \\
\hline & $\mathrm{Zn}$ & 0.4 & 0.3 & 0.3 \\
\hline & $\mathrm{Zr}$ & 0.8 & 0.8 & 1.4 \\
\hline & $\mathrm{U}$ & 0.4 & 0.8 & 1.4 \\
\hline & $\mathrm{Pu}$ & 0.4 & 0.4 & 0.7 \\
\hline & Am & 0.4 & 0.5 & 0.5 \\
\hline \multirow[t]{14}{*}{ Alkaline Supernate } & $\mathrm{Ce}$ & 78 & 370 & 499 \\
\hline & $\mathrm{Cs}$ & 0.8 & 0.3 & 0.3 \\
\hline & $\mathrm{Sr}$ & $<0.1$ & 0.2 & 0.3 \\
\hline & $\mathrm{Tc}$ & 41 & 111 & 196 \\
\hline & $\mathrm{Y}$ & 15 & 45 & 89 \\
\hline & $\mathrm{Cr}$ & 0.8 & 0.7 & 0.5 \\
\hline & Co & 0.1 & 1.0 & 0.8 \\
\hline & $\mathrm{Fe}$ & $<0.1$ & $<0.1$ & 0.2 \\
\hline & $\mathrm{Mn}$ & 1.6 & 1.6 & 2.1 \\
\hline & $\mathrm{Zn}$ & 1.0 & 0.4 & 0.5 \\
\hline & $\mathrm{Zr}$ & 1.1 & 0.8 & 0.8 \\
\hline & $\mathrm{U}$ & 49 & 183 & 291 \\
\hline & $\mathrm{Pu}$ & 0.8 & 0.5 & 1.0 \\
\hline & $\mathrm{Am}$ & 49 & 355 & 472 \\
\hline
\end{tabular}


j. Aliquat ${ }^{\mathrm{TM}}$ 336. Five grams of Aliquat ${ }^{\mathrm{TM}} 336$, (tricaprylmethylammonium chloride) obtained from Aldrich Chemical Co., was diluted with $10 \mathrm{ml}$ of hexane, after which $17.32 \mathrm{~g}$ of Ambersorb ${ }^{\text {TM }} 563$ porous carbon beads, obtained from Rohm \& Haas Co., Philadelphia, PA, was added to absorb the liquid. The cyclohexane evaporated to yield $26.2 \mathrm{~g}$ of the loaded Ambersorb $^{\mathrm{TM}}$ beads.

Plutonium is moderately sorbed from acidic solution. In basic solution, technetium is strongly sorbed with high selectivity and only uranium is cosorbed to a significant extent. This extractant demonstrates an outstanding ability to selectively recover technetium from alkaline supernate solution.

Table III.B.6.j. Distribution of 14 Elements into Aliquat ${ }^{\mathrm{TM}} 336$ from Two Simulant Solutions for Hanford HLW Tank 102-SY

\begin{tabular}{|c|c|c|c|c|}
\hline Medium & Absorber & 30-min Kd & 2-h Kd & 6-h Kd \\
\hline \multirow[t]{14}{*}{ Acid-Dissolved Sludge } & $\mathrm{Ce}$ & 0.4 & 0.6 & 0.6 \\
\hline & Cs & 0.3 & 0.3 & 0.2 \\
\hline & $\mathrm{Sr}$ & 0.4 & 0.3 & 0.3 \\
\hline & $\mathrm{Tc}$ & 0.7 & 0.8 & 1.0 \\
\hline & $\mathrm{Y}$ & 0.3 & 0.3 & 0.2 \\
\hline & $\mathrm{Cr}$ & $<0.1$ & $<0.1$ & 0.1 \\
\hline & Co & 0.6 & 0.3 & $<0.1$ \\
\hline & $\mathrm{Fe}$ & 0.2 & 0.3 & 0.5 \\
\hline & $\mathrm{Mn}$ & $<0.1$ & $<0.1$ & $<0.1$ \\
\hline & $\mathrm{Zn}$ & 0.3 & 0.1 & 0.1 \\
\hline & $\mathrm{Zr}$ & 0.8 & 0.4 & 0.6 \\
\hline & $\mathrm{U}$ & 0.5 & 1.1 & 1.7 \\
\hline & $\mathrm{Pu}$ & 2.3 & 5.7 & 14 \\
\hline & $\mathrm{Am}$ & 0.2 & 0.2 & 0.2 \\
\hline \multirow[t]{14}{*}{ Alkaline Supernate } & $\mathrm{Ce}$ & 1.0 & 1.6 & 2.0 \\
\hline & Cs & 1.8 & 2.2 & 2.1 \\
\hline & $\mathrm{Sr}$ & 0.3 & 0.2 & 0.3 \\
\hline & $\mathrm{Tc}$ & 295 & 444 & 572 \\
\hline & $Y$ & 0.3 & 0.4 & 0.2 \\
\hline & $\mathrm{Cr}$ & 0.1 & 0.1 & 0.1 \\
\hline & Co & 0.2 & 0.1 & $<0.1$ \\
\hline & $\mathrm{Fe}$ & 0.2 & 0.3 & 0.3 \\
\hline & $\mathrm{Mn}$ & 0.3 & 0.3 & 0.3 \\
\hline & $\mathrm{Zn}$ & $<0.1$ & $<0.1$ & $<0.1$ \\
\hline & $\mathrm{Zr}$ & $<0.1$ & 0.1 & 0.1 \\
\hline & U & 3.2 & 5.5 & 7.9 \\
\hline & $\mathrm{Pu}$ & 0.1 & 0.2 & 0.4 \\
\hline & $\mathrm{Am}$ & 0.4 & 0.6 & 0.6 \\
\hline
\end{tabular}


k. Czech Cobalt Dicarbollide. A 93.6-mg portion of cobalt dicarbollide prepared in the Czech Republic was dissolved in $2.323 \mathrm{~g}$ of nitrobenzene and $1 \mathrm{ml}$ of ethanol, alter which $2.628 \mathrm{~g}$ of Ambersorb 563 porous carbon beads, obtained from Rohm \& Haas Co.,

Philadelphia, PA, was added to absorb the liquid. The ethanol evaporated to yield $4.48 \mathrm{~g}$ of the loaded, dry-appearing Ambersorb ${ }^{T M}$ beads.

Technetium sorbs moderately, with slow sorption kinetics, from acidic and basic solution.

Table III.B.6.k. Distribution of 14 Elements into Czech Cobalt Dicarbollide from Two Simulant Solutions for Hanford HLW Tank 102-SY

\begin{tabular}{|c|c|c|c|c|}
\hline Medium & Absorber & 30-min Kd & 2-h Kd & 6-h Kd \\
\hline \multirow[t]{14}{*}{ Acid-Dissolved Sludge } & $\mathrm{Ce}$ & 0.5 & 0.1 & 0.1 \\
\hline & Cs & $<0.1$ & $<0.1$ & 0.3 \\
\hline & $\mathrm{Sr}$ & 0.6 & 0.5 & 0.9 \\
\hline & $\mathrm{Tc}$ & 0.9 & 2.9 & 7.0 \\
\hline & $\mathrm{Y}$ & 0.4 & 0.4 & 0.5 \\
\hline & $\mathrm{Cr}$ & 0.2 & 0.4 & 0.4 \\
\hline & Co & 0.2 & 0.3 & 0.3 \\
\hline & $\mathrm{Fe}$ & $<0.1$ & $<0.1$ & $<0.1$ \\
\hline & $\mathrm{Mn}$ & 0.2 & 0.7 & 0.6 \\
\hline & $\mathrm{Zn}$ & 0.3 & 0.3 & 0.4 \\
\hline & $\mathrm{Zr}$ & 0.5 & 0.7 & 0.8 \\
\hline & $\mathrm{U}$ & 1.2 & 0.9 & 1.1 \\
\hline & $\mathrm{Pu}$ & $<0.1$ & 0.3 & 0.4 \\
\hline & $\mathrm{Am}$ & 0.2 & 0.3 & 0.3 \\
\hline \multirow[t]{14}{*}{ Alkaline Supernate } & $\mathrm{Ce}$ & 0.7 & 1.4 & 1.2 \\
\hline & $\mathrm{Cs}$ & 0.8 & 1.0 & 0.8 \\
\hline & $\mathrm{Sr}$ & $<0.1$ & 0.5 & 0.7 \\
\hline & $\mathrm{Tc}$ & 0.6 & 3.8 & 13 \\
\hline & $\mathrm{Y}$ & $<0.1$ & $<0.1$ & $<0.1$ \\
\hline & $\mathrm{Cr}$ & 0.5 & 0.9 & 1.1 \\
\hline & $\mathrm{Co}$ & 0.4 & 0.8 & 0.7 \\
\hline & $\mathrm{Fe}$ & 0.2 & $<() .1$ & 0.2 \\
\hline & $\mathrm{Mn}$ & 0.2 & 0.3 & 0.2 \\
\hline & $\mathrm{Zn}$ & 0.5 & 0.3 & 0.2 \\
\hline & $\mathrm{Zr}$ & 0.2 & 0.7 & 1.0 \\
\hline & U & 0.9 & 0.9 & 0.8 \\
\hline & $\mathrm{Pu}$ & 0.1 & $<() .1$ & $<0.1$ \\
\hline & Am & 0.2 & 0.1 & 0.1 \\
\hline
\end{tabular}


I. INC-Synthesized Cobalt Dicarbollide. A $98.1-\mathrm{mg}$ portion of cobalt dicarbollide prepared by the LANL Isotope and Nuclear Chemistry (INC) Division was dissolved in $1.816 \mathrm{~g}$ of nitrobenzene and $1 \mathrm{ml}$ of ethanol. Then $2.270 \mathrm{~g}$ of Ambersorb ${ }^{\mathrm{TM}} 563$ porous carbon beads, obtained from Rohm \& Haas Co., Philadelphia, PA, was added to absorb the liquid. The ethanol evaporated to yield $3.64 \mathrm{~g}$ of the loaded, dry-appearing Ambersorb ${ }^{\mathrm{TM}}$ beads.

Moderate sorption of technetium and low sorption of cesium occur from acidic and basic solution, also with slow sorption kinetics.

Table III.B.6.I. Distribution of 14 Elements into INC-Synthesized Cobalt Dicarbollide from Two Simulant Solutions for Hanford HLW Tank 102-SY

\begin{tabular}{|c|c|c|c|c|}
\hline Medium & Absorber & 30-min Kd & 2-h Kd & 6-h Kd \\
\hline \multirow[t]{14}{*}{ Acid-Dissolved Sludge } & $\mathrm{Ce}$ & $<0.1$ & $<0.1$ & $<0.1$ \\
\hline & $\mathrm{Cs}$ & 1.5 & 1.8 & 1.2 \\
\hline & $\mathrm{Sr}$ & 0.6 & 0.5 & 0.6 \\
\hline & $\mathrm{Tc}$ & 1.6 & 3.4 & 7.7 \\
\hline & $\mathrm{Y}$ & 0.6 & 0.2 & 0.2 \\
\hline & $\mathrm{Cr}$ & 0.1 & $<0.1$ & 0.1 \\
\hline & Co & 0.2 & 0.5 & 0.2 \\
\hline & $\mathrm{Fe}$ & 0.1 & $<0.1$ & $<0.1$ \\
\hline & $\mathrm{Mn}$ & $<0.1$ & 0.2 & 0.3 \\
\hline & $\mathrm{Zn}$ & 0.2 & 0.3 & 0.3 \\
\hline & $\mathrm{Zr}$ & 0.4 & 0.4 & 0.7 \\
\hline & $U$ & 0.7 & 0.7 & 0.5 \\
\hline & $\mathrm{Pu}$ & $<0.1$ & $<0.1$ & 0.2 \\
\hline & Am & 0.1 & 0.1 & 0.2 \\
\hline \multirow[t]{14}{*}{ Alkaline Supernate } & $\mathrm{Cc}$ & 0.9 & 0.8 & 0.6 \\
\hline & $\mathrm{Cs}$ & 3.1 & 2.0 & 1.2 \\
\hline & $\mathrm{Sr}$ & 0.6 & 0.5 & 0.2 \\
\hline & $\mathrm{Tc}$ & 1.9 & 7.2 & 44 \\
\hline & Y & $<0.1$ & $<0.1$ & $<0.1$ \\
\hline & $\mathrm{Cl}$ & 0.8 & 1.2 & 0.6 \\
\hline & $\mathrm{Co}$ & 0.6 & 1.0 & 0.5 \\
\hline & $\mathrm{Fc}$ & 0.2 & 0.5 & 0.4 \\
\hline & $\mathrm{Mn}$ & 0.2 & 0.1 & 0.1 \\
\hline & $\mathrm{Zn}$ & $<() .1$ & 0.1 & 0.1 \\
\hline & $\mathrm{Zr}$ & 1.1 & 0.9 & 0.8 \\
\hline & $\mathrm{U}$ & 0.5 & 0.9 & 0.9 \\
\hline & $\mathrm{Pu}$ & 0.3 & 0.1 & ().2 \\
\hline & $\mathrm{Am}$ & $<0.1$ & 0.1 & 0.1 \\
\hline
\end{tabular}


7. SNL Absorbers. The materials designated as SNL/CST are a new class of inorganic ionexchangers called crystalline silico-titanates (CSTs). These materials, jointly invented by SNL and Texas A\&M University (a patent application has been filed), show significant potential for removal of cesium from defense wastes that are $>5 \mathrm{M} \mathrm{Na}^{+}$and $>1 \mathrm{M} \mathrm{OH}$. The numerical values in the absorber designations represent different synthesis procedures. CST materials are very fine powders composed of cuboidal crystals with particle sizes of several hundred Angstroms.

Screening studies on these materials at SNL have indicated the selectivity for cesium removal is SNL/CST35 < SNL/CST48 < SNL/CST68 < SNL/CST69 < SNL/CST84 < SNL/CST1 11. SNL/CST84 and SNL/CST111 are markedly different from the other absorbers in terms of their synthesis; they contain a dopant, with the dopant/CST ratio highest for SNL/CST111. SNL investigations have demonstrated that the presence of the dopant significantly increases the value of the distribution coefficient, as well as the structural stability of the CST material in highly alkaline solutions.

As discussed in Section II.D., the unusually large variation in $\mathrm{Kd}$ values obtained with CST absorbers indicates that submicron CST particles sometimes contaminated the solution taken for gamma spectrometric assay. The inadvertent introduction of particles into the assaly solution would, however, always cause us to understate the actual $\mathrm{Kds}$ for removing cesium from solution. Our reported Kd values for cesium sorption from the CST materials may therefore be considered as minimum values.

In a few other cases, the $\mathrm{Kd}$ values for non-cesium elements decrease with increasing contact time. SNL personnel suggested that this could be caused by a small amount of an amorphous phase in the crystalline material, which appears to preferentially sorb certain non-cesium elements. However, because the amorphous phase slowly dissolves, some of the initially sorbed element is released, causing the apparent $\mathrm{Kd}$ value to decrease with increased contact time.

a. SNL/CST35 Crystalline Silico-Titanate. Cesium is very strongly and quite selectively sorbed from acidic solution; only zirconium cosorbs to a significant extent. Cesium is sorbed even more strongly from basic solution, and uranium also is sorbed at useful levels from this medium. 
Table III.B.7.a. Distribution of 14 Elements onto SNL/CST3.5 from Two Simulant Solutions for Hanford HLW Tank 102-SY

\begin{tabular}{|c|c|c|c|c|}
\hline Medium & Absorber & $30-\min \mathrm{Kd}$ & 2-h Kd & 6-h Kd \\
\hline \multirow[t]{14}{*}{ Acid-Dissolved Sludge } & $\mathrm{Ce}$ & 0.6 & 0.7 & 0.7 \\
\hline & Cs & 4485 & 1011 & 3416 \\
\hline & $\mathrm{Sr}$ & $<0.1$ & $<0.1$ & $<0.1$ \\
\hline & $\mathrm{Tc}$ & 0.9 & 0.5 & 0.5 \\
\hline & $\mathrm{Y}$ & 0.3 & 0.4 & 0.4 \\
\hline & $\mathrm{Cr}$ & 0.4 & 0.4 & 0.4 \\
\hline & $\mathrm{Co}$ & 0.6 & 0.7 & 0.5 \\
\hline & $\mathrm{Fe}$ & 0.8 & 0.5 & 0.8 \\
\hline & $\mathrm{Mn}$ & $<0.1$ & $<0.1$ & 0.1 \\
\hline & $\mathrm{Zn}$ & 0.5 & 0.3 & 0.3 \\
\hline & $\mathrm{Zr}$ & 8.3 & 26 & 76 \\
\hline & U & 0.9 & 0.5 & 0.5 \\
\hline & $\mathrm{Pu}$ & 2.4 & 2.6 & 2.4 \\
\hline & Am & $<0.1$ & 0.1 & $<0.1$ \\
\hline \multirow[t]{14}{*}{ Alkaline Supernate } & $\mathrm{Ce}$ & 2.9 & 2.1 & 6.7 \\
\hline & Cs & 13158 & 12421 & 4195 \\
\hline & $\mathrm{Sr}$ & 1.6 & 1.3 & 3.3 \\
\hline & Tc & 1.2 & 1.7 & 5.6 \\
\hline & $\mathrm{Y}$ & 2.5 & 1.7 & 0.9 \\
\hline & $\mathrm{Cr}$ & 1.1 & 1.9 & 9.6 \\
\hline & $\mathrm{Co}$ & 0.7 & 0.2 & 0.2 \\
\hline & $\mathrm{Fe}$ & 0.9 & 2.2 & 2.5 \\
\hline & $\mathrm{Mn}$ & 0.5 & 0.1 & 0.1 \\
\hline & $\mathrm{Zn}$ & 1.5 & 1.1 & 0.9 \\
\hline & $\mathrm{Zr}$ & 0.5 & 1.1 & 3.6 \\
\hline & U & 25 & 4.5 & 54 \\
\hline & $\mathrm{Pu}$ & 0.8 & 1.5 & 1.5 \\
\hline & Am & 1.3 & 1.1 & 0.7 \\
\hline
\end{tabular}


b. SNL/CST48 Crystalline Silico-'Titanate. Cesium is very strongly and selectively sorbed from acidic solution; only zirconium cosorbs to a significant extent. Cesium sorbs even more strongly from basic solution, although the selectivity is not as high.

Table III.B.7.b. Distribution of 14 Elements onto SNL/CST48 from Two Simulant Solutions for Hanford HLW Tank 102-SY

\begin{tabular}{|c|c|c|c|c|}
\hline Medium & Absorber & 30-min Kd & 2-h Kd & 6-h Kd \\
\hline \multirow[t]{14}{*}{ Acid-Dissolved Sludge } & $\mathrm{Ce}$ & 0.6 & 0.4 & 0.6 \\
\hline & Cs & 794 & 758 & 1180 \\
\hline & $\mathrm{Sr}$ & $<0.1$ & $<0.1$ & $<0.1$ \\
\hline & $\mathrm{Tc}$ & 0.8 & 0.5 & 0.6 \\
\hline & Y & 0.7 & 0.4 & 0.5 \\
\hline & $\mathrm{Cr}$ & 0.3 & 0.3 & 0.3 \\
\hline & $\mathrm{Co}$ & 0.9 & 0.4 & 0.6 \\
\hline & $\mathrm{Fe}$ & 0.7 & 1.0 & 1.0 \\
\hline & $\mathrm{Mn}$ & $<0.1$ & $<0.1$ & $<0.1$ \\
\hline & $\mathrm{Zn}$ & 0.3 & 0.2 & 0.2 \\
\hline & $\mathrm{Zr}$ & 15 & 49 & 51 \\
\hline & $\mathrm{U}$ & 1.5 & 1.1 & 1.2 \\
\hline & $\mathrm{Pu}$ & 2.6 & 3.2 & 4.4 \\
\hline & $\mathrm{Am}$ & $<0.1$ & 0.2 & 0.1 \\
\hline \multirow[t]{14}{*}{ Alkaline Supernate } & $\mathrm{Ce}$ & 6.4 & 8.6 & 17 \\
\hline & $\mathrm{Cs}$ & 3951 & 2820 & 1030 \\
\hline & $\mathrm{Sr}$ & 1.8 & 1.2 & 1.2 \\
\hline & $\mathrm{Tc}$ & 1.6 & 1.5 & 2.0 \\
\hline & Y & 6.0 & 7.0 & 5.3 \\
\hline & $\mathrm{Cr}$ & 1.3 & 1.7 & 6.0 \\
\hline & $\mathrm{Co}$ & 4.5 & 4.6 & 2.0 \\
\hline & $\mathrm{Fe}$ & 1.2 & 1.5 & 1.8 \\
\hline & $\mathrm{Mn}$ & 2.0 & 1.7 & 1.2 \\
\hline & $\mathrm{Zn}$ & 23 & 32 & 34 \\
\hline & $\mathrm{Zr}$ & 0.7 & 0.8 & 0.8 \\
\hline & $\mathrm{U}$ & 16 & 30 & 37 \\
\hline & $\mathrm{Pu}$ & $<0.1$ & $<0.1$ & 0.1 \\
\hline & $\mathrm{Am}$ & 9.4 & 13 & 15 \\
\hline
\end{tabular}


c. SNL/CST68 Crystalline Silico-Titanate. Cesium is very strongly and selectively sorbed from acidic solution; only zirconium is cosorbed to a significant extent. Cesium is sorbed even more strongly from basic solution, although the selectivity is not as high. The decreasing $\mathrm{Kd}$ values for cesium may be real, or they may reflect incomplete filtration of the submicron absorber particles from the assiay solutions.

Table III.B.7.c. Distribution of 14 Elements onto SNL/CST68 from Two Simulant Solutions for Hanford HLW Tank 102-SY

\begin{tabular}{|c|c|c|c|c|}
\hline Medium & Absorber & 30-min Kd & 2-h Kd & 6-h Kd \\
\hline \multirow[t]{14}{*}{ Acid-Dissolved Sludge } & $\mathrm{Ce}$ & 0.1 & 0.4 & 0.7 \\
\hline & $\mathrm{Cs}$ & 688 & 2308 & 1049 \\
\hline & $\mathrm{Sr}$ & $<0.1$ & $<0.1$ & $<0.1$ \\
\hline & $\mathrm{Tc}$ & 0.6 & 0.4 & 0.8 \\
\hline & $Y$ & 0.5 & 0.2 & 0.3 \\
\hline & $\mathrm{Cr}$ & 0.1 & 0.4 & 0.4 \\
\hline & $\mathrm{Co}$ & 0.5 & 0.3 & 0.5 \\
\hline & $\mathrm{Fe}$ & 0.7 & 0.7 & 0.9 \\
\hline & $\mathrm{Mn}$ & $<0.1$ & 0.3 & 0.1 \\
\hline & $\mathrm{Zn}$ & 0.2 & 0.4 & 0.3 \\
\hline & $\mathrm{Zr}$ & 31 & 119 & 103 \\
\hline & $\mathrm{U}$ & 1.1 & 1.2 & 1.0 \\
\hline & $\mathrm{Pu}$ & 3.9 & 4.4 & 5.3 \\
\hline & $\mathrm{Am}$ & 0.4 & 0.1 & 0.1 \\
\hline \multirow[t]{14}{*}{ Alkaline Supernate } & $\mathrm{Ce}$ & 8.6 & 16 & 38 \\
\hline & $\mathrm{Cs}$ & 4072 & 2453 & 722 \\
\hline & $\mathrm{Sr}$ & 1.7 & 1.6 & 1.3 \\
\hline & $\mathrm{Tc}$ & 1.5 & 1.8 & 1.7 \\
\hline & $Y$ & 7.4 & 9.7 & 7.5 \\
\hline & $\mathrm{Cr}$ & 1.7 & 2.4 & 6.6 \\
\hline & Co & 5.3 & 5.4 & 2.3 \\
\hline & $\mathrm{Fe}$ & 0.9 & 1.7 & 2.2 \\
\hline & $\mathrm{Mn}$ & 1.9 & 1.9 & 1.6 \\
\hline & $\mathrm{Zn}$ & 33 & 44 & 43 \\
\hline & $\mathrm{Zr}$ & 1.0 & 1.2 & $(1.9$ \\
\hline & $\mathrm{U}$ & 23 & 38 & 45 \\
\hline & $\mathrm{Pu}$ & 0.6 & 0.5 & 0.3 \\
\hline & $\mathrm{Am}$ & 15 & 20 & 20 \\
\hline
\end{tabular}


d. SNL/CST69 Crystalline Silleo-'Titunate. Cesium is very strongly and selectively sorbed from acidic solution; only zirconium cosorbs to a significant extent. Cesium is sorbed even more strongly from basic solution, aithough the selectivity is not as high.

Table III.B.7.d. Distribution of 14 Elements onto SNL/CST69 from Two Simulant Solutions for Hanford HLW Tank 102-SY

\begin{tabular}{|c|c|c|c|c|}
\hline Medium & Absorber & 30-min Kd & 2-h Kd & 6.h Kd \\
\hline \multirow[t]{14}{*}{ Acid-Dissolved Sludge } & $\mathrm{Ce}$ & 0.5 & 0.6 & 0.7 \\
\hline & $\mathrm{Cs}$ & 2884 & 2279 & 3252 \\
\hline & $\mathrm{Sr}$ & $<0.1$ & $<0.1$ & $<0.1$ \\
\hline & $\mathrm{Tc}$ & 1.1 & 0.8 & 1.1 \\
\hline & $\mathrm{Y}$ & 0.4 & (0.4 & 0.2 \\
\hline & $\mathrm{Cr}$ & 0.6 & 0.7 & 0.6 \\
\hline & $\mathrm{Co}$ & 0.4 & 0.4 & 0.4 \\
\hline & $\mathrm{Fe}$ & 0.4 & 0.6 & 0.7 \\
\hline & $\mathrm{Mn}$ & $<0.1$ & $<0.1$ & $<0.1$ \\
\hline & $\mathrm{Zn}$ & 0.4 & 0.4 & 0.4 \\
\hline & $\mathrm{Zr}$ & 91 & $14^{i 1}$ & 115 \\
\hline & $\mathrm{U}$ & 0.7 & 1.1 & 1.2 \\
\hline & $\mathrm{Pu}$ & 3.4 & 3.8 & 4.5 \\
\hline & Am & 0.6 & 0.3 & 0.3 \\
\hline \multirow[t]{14}{*}{ Alkaline Supernate } & $\mathrm{Ce}$ & 4.0 & 4.2 & 14 \\
\hline & $\mathrm{Cs}$ & 8879 & 10844 & 20880 \\
\hline & $\mathrm{Sr}$ & 3.4 & 2.9 & 6.6 \\
\hline & $\mathrm{Tc}$ & 4.9 & 6.7 & 12 \\
\hline & $Y$ & 1.8 & 1.0 & 1.6 \\
\hline & $\mathrm{Cr}$ & 2.8 & 3.8 & 14 \\
\hline & Co & $<0.1$ & $<0.1$ & $<0.1$ \\
\hline & $\mathrm{Fe}$ & 2.2 & 2.0 & 2.2 \\
\hline & $\mathrm{Mn}$ & 0.4 & 0.4 & 0.4 \\
\hline & $\mathrm{Zn}$ & 2.0 & 2.9 & 3.7 \\
\hline & $\mathrm{Zr}$ & 1.7 & 2.5 & 5.5 \\
\hline & $\mathrm{U}$ & 21 & 33 & 43 \\
\hline & $\mathrm{Pu}$ & 0.7 & 0.7 & 1.0 \\
\hline & Am & 2.5 & 3.0 & 3.3 \\
\hline
\end{tabular}


e. SNI /CST84 Crystalline Silico-'Titunate. Cesium is very strongly and selectively sorbed from acidic solution; only zirconium coserbs to a significant extent. The decreasing Kd values for zirconium from acidic solution (while cesium $\mathrm{Kd}$ values increase) may reflect the slow dissolution of an amorphous phase that preferentially sorbs zirconium. Cesium is sorbed well from basic solution, although the selectivity is not as high.

Table III.B.7.e. Distribution of 14 Elements onto SNL/CST84 liom Two Simulant Solutions for Hanford HLW Tank 102-SY

\begin{tabular}{|c|c|c|c|c|}
\hline Medium & Absorber & 30-min Kd & 2.h Kd & 6-h Kd \\
\hline \multirow[t]{14}{*}{ Acid-Dissolved Sludge } & $\mathrm{Ce}$ & 0.4 & 0.1 & 0.5 \\
\hline & $\mathrm{Cs}$ & 1849 & 8096 & 11840 \\
\hline & $\mathrm{Sr}$ & $<0.1$ & $<0.1$ & $<0.1$ \\
\hline & $T c$ & 0.8 & 0.4 & 0.7 \\
\hline & $Y$ & $<0.1$ & 0.1 & 0.2 \\
\hline & $\mathrm{Cr}$ & 0.4 & 0.6 & 0.6 \\
\hline & $\mathrm{Co}$ & 0.6 & 0.3 & 0.4 \\
\hline & $\mathrm{Fe}$ & 0.4 & 0.2 & 0.4 \\
\hline & $\mathrm{Mn}$ & $<0.1$ & 0.1 & $<0.1$ \\
\hline & $\mathrm{Zn}$ & 0.4 & 0.3 & 0.2 \\
\hline & $\mathrm{Zr}$ & 70 & 87 & 19 \\
\hline & U & 0.6 & 0.9 & 0.5 \\
\hline & $\mathrm{Pu}$ & 2.0 & 1.8 & 1.9 \\
\hline & $\mathrm{Am}$ & 0.1 & $<0.1$ & $<0.1$ \\
\hline \multirow[t]{14}{*}{ Alkaline Supernate } & $\mathrm{Ce}$ & 3.5 & 4.8 & 14 \\
\hline & Cs & 5484 & 3953 & 3098 \\
\hline & $\mathrm{Sr}^{r}$ & 2.2 & 2.0 & 4.4 \\
\hline & $\mathrm{Tc}$ & 1.5 & 1.9 & 4.6 \\
\hline & $Y$ & 1.3 & 1.1 & 1.1 \\
\hline & $\mathrm{Cr}$ & 1.4 & 1.7 & 8.8 \\
\hline & $\mathrm{Co}$ & 0.3 & 0.2 & 0.1 \\
\hline & $\mathrm{Fe}$ & 0.6 & 1.2 & 1.4 \\
\hline & $\mathrm{Mn}$ & 0.9 & 0.6 & 0.5 \\
\hline & $\mathrm{Zn}$ & 1.0 & 1.4 & 2.1 \\
\hline & $\mathrm{Zr}$ & 0.9 & 1.1 & 3.0 \\
\hline & $\mathrm{U}$ & 13 & 19 & 22 \\
\hline & $\mathrm{Pu}$ & 0.1 & 0.3 & 0.2 \\
\hline & Am & 2.9 & 4.1 & 5.8 \\
\hline
\end{tabular}


f. SNL/CST111 Crystulline Sillico-Titanate. Cesium is very strongly and sclectively sorbed from acidic solution; only zirconium cosorbs to a significant extent. Cesium is sorbed well from basic solution, although the selectivity is not as high. The decrealsing Kol vallues for cesium from basic solution may be real, or they may reflect incomplete filtration of the submicron absorber particles from the assaly solutions.

Table III.B.7.f. Distribution of 14 Elements onto SNL/CST11/ from Two Simulant Solutions for Hanford HLW Tank 102-SY

\begin{tabular}{|c|c|c|c|c|}
\hline Medium & Absorber & 30-min Kd & 2-h Kd & 6-h Kd \\
\hline \multirow[t]{14}{*}{ Acid-Dissolved Sludge } & $\mathrm{Ce}$ & 1.0 & 0.9 & 1.0 \\
\hline & Cs & 3420 & 5021 & 5305 \\
\hline & $\mathrm{Sr}$ & 0.6 & 0.4 & 0.5 \\
\hline & $\mathrm{Tc}$ & 0.6 & 0.7 & 0.7 \\
\hline & $Y$ & 0.3 & 0.3 & 0.3 \\
\hline & $\mathrm{Cr}$ & 1.0 & 1.1 & 1.2 \\
\hline & Co & $<0.1$ & $<0.1$ & $<0.1$ \\
\hline & $\mathrm{Fe}$ & 0.6 & 0.6 & 0.7 \\
\hline & $\mathrm{Mn}$ & 0.1 & 0.3 & 0.1 \\
\hline & $\mathrm{Zn}$ & 0.8 & 0.6 & 0.6 \\
\hline & $\mathrm{Zr}$ & 11 & 14 & 18 \\
\hline & U & 0.7 & 1.3 & 1.5 \\
\hline & $\mathrm{Pu}$ & 3.1 & 3.5 & 3.8 \\
\hline & Am & 0.2 & 0.3 & 0.3 \\
\hline \multirow[t]{14}{*}{ Alkaline Supernate } & $\mathrm{Ce}$ & 17 & 32 & 57 \\
\hline & Cs & 4939 & 2713 & 1864 \\
\hline & $\mathrm{Sr}$ & 4.2 & 4.2 & 3.5 \\
\hline & Tc & 1.5 & 1.8 & 1.2 \\
\hline & $Y$ & 4.4 & 4.7 & 4.8 \\
\hline & $\mathrm{Cr}$ & 2.1 & 1.9 & 1.7 \\
\hline & Co & 1.0 & 0.8 & 0.4 \\
\hline & $\mathrm{Fe}$ & 0.6 & 1.6 & 1.9 \\
\hline & $\mathrm{Mn}$ & 1.2 & 1.6 & 1.5 \\
\hline & $\mathrm{Zn}$ & 2.3 & 3.2 & 5.1 \\
\hline & $\mathrm{Zr}$ & 0.9 & 1.0 & 0.6 \\
\hline & U & 15 & 21 & 2.5 \\
\hline & $\mathrm{Pu}$ & 0.2 & 0.8 & 0.4 \\
\hline & $\mathrm{Am}$ & 25 & 52 & 107 \\
\hline
\end{tabular}


g. SNL/H'TO Amorphous Hydrous 'Ti(), As expected, the behavior of this hydrous titunium oxide is very different from that of the erystalline silico-titanates. Cerium, annericium, uranium and yttrium sorb well, whereas chromium, zinc, cesium, and zirconium sorb moderately from basic solution.

Table III.B.7.g. Distribution of 14 Elements onto SNL/HTO from Simulated Alkalline Supernate Solution for Hanford HLW Tank 102-SY

\begin{tabular}{lllll}
\hline Medium & Absorber & $30-\mathrm{min} \mathrm{Kd}$ & $2-\mathrm{h} \mathrm{Kd}$ & $6-\mathrm{h}$ Kd
\end{tabular}

Acid-Dissolved Sludge

At the recommendation of SNL. personnel, SNL/HTO was not tested in acid.

\begin{tabular}{ccccc} 
Alkiline Supernate & Ce & 265 & 320 & 511 \\
& Cs & 10 & 8.9 & 8.5 \\
Sr & 1.5 & 1.4 & 3.6 \\
Tc & 1.3 & 1.7 & 4.3 \\
Y & 53 & 59 & 52 \\
Cr & 5.9 & 7.8 & 21 \\
Co & 0.9 & 0.7 & 0.4 \\
& $\mathrm{Fc}$ & 2.2 & 3.1 & 3.9 \\
$\mathrm{Mn}$ & 1.2 & 0.3 & 0.4 \\
$\mathrm{Zn}$ & 18 & 18 & 19 \\
$\mathrm{Zr}$ & 1.7 & 2.7 & 6.3 \\
$\mathrm{U}$ & 52 & 69 & 90 \\
$\mathrm{Pu}$ & 1.5 & 1.8 & 2.4 \\
$\mathrm{Am}$ & 229 & 264 & 168 \\
\hline
\end{tabular}




\section{ELUTION CONSIDERATIONS}

The purpose of this study was to screen a large number of candidate absorbers and identify those that show promise for specific elements. Although elution was not a part of our study, we recognize that elution of the sorbed species from the selected absorbers is essential if the absorbers are to be reused in a process. We therefore plan to investigate the elution characteristics of selected a sorbers in the next phase of this study.

We can, however, offer some general comments about elution. Elution often can be achieved by (1) acidity changes, (2) oxidation state changes, (3) complexation, or (4) displacement. Because our present study reports distribution data from both acidic and basic simulant solutions, it often indicates whether acidity changes might elute a given species. In cases in which a specific oxidation state is strongly sorbed on an absorber, another oxidation state of that same element may not be sorbed, and conversion to this other oxidation state may achieve elution. In still other cases, the sorbed species may be capable of forming a strong complex with certain complexants, which can be used as eluting agents. Finally, sorbed species can sometimes be displaced by loading the absorber to capacity with another ion that is more strongly sorbed.

Because elution operations usually generate additional waste, we plan to emphasize waste minimization in the elution options we evaluate in the next phase of this study. One option worth considering is that of not eluting the sorbed species from the absorber. For example, this option is attractive in the case of the absorbers developed by SNL and Texas A\&M because cesium appears to be irreversibly sorbed with very high $\mathrm{Kd}$ values on silico-titanates. Moreover, the silicotitanates provide an inorganic matrix that can be pressed into dense pellets suitable for long-term storage. In this case, elution may be unwise and unnecessary.

Ammonium molybdophosphate (AMP) offers cesium $\mathrm{Kd}$ values from acidic and basic solution nearly as high as those on silico-titanates. Moreover, cesium can be eluted from AMP with $5 \mathrm{M}$ ammonium chloride. ${ }^{15} \mathrm{~A}$ very attractive possibility is eluting cesium with ammonium chloride, distilling and colleciing the water, followed by sublimation of ammonium chloride at $355^{\prime \prime} \mathrm{C}$, but not cesium chloride, whose sublimation temperature is $1290^{\circ} \mathrm{C}$. The water and ammonium chloride could then be combined and recycled to elute the next batch of cesium, which could provide highly concentrated cesium, while generating zero secondary waste in the elution step. This is an example of innovative chemistry that can minimize secondary-waste generation.

\section{FUTURE STUDIES}

Our screening study has met its objective of identifying specific absorbers that seem capable of partitioning targeted elements from Hanford HLW Tank 102-SY. The best absorbers from this study and perhaps a few promising new materials should be evaluated with simulant compositions that represent other Hanford tanks, especially those on the safety watch-list. The 14 elements whose distributions have been measured should be reviewed to determine if any should he deleted or if additional elements should be incorporated in future measurements. Finally, radioactive tracers, which provide a rapid, reliable, and inexpensive way to obtain large amounts of distribution data, should be used in future studies. 
The effect of ionizing radiation is an important factor to consider when selecting materials to be used in processing radioactive waste solutions. The effect of radiation on the most promising absorbers from our present and future studies should be determined, if such information is not already available, ${ }^{16}$ before any large-scale recovery flowsheet is selected.

\section{CONCLUSIONS}

1. Our screening study of many absorbers from realistic simulants has met our initial objective of identifying numerous promising absorbers. Many of the separations required for the remediation of Hanford HLW Tank 102-SY can be achieved using available absorbers and existing, proven technology.

2. Radioactive tracers, as used in our study, provide a reliable and cost-effective way to rapidly obtain large amounts of distribution data. Follow-on studies should measure the distribution of key elements from other Hanford waste tank compositions onto the most promising absorbers identified by this study.

3. The performance of the 60 absorbers tested $v$ ith these complex, high-salt media is often much different from published results for similar absorbers tested in relatively clean systems.

4. Elements for which good-to-excellent sorption has been demonstrated from simulated aciddissolved sludge are cerium, cesium, technetium, zirconium, uranium, and plutonium. Elements that show low sorption from acidic solution are strontium, yttrium, chromium, cobalt, iron, manganese, zinc, and americium.

5. Elements for which good-to-excellent sorption has been demonstrated from alkaline supernate simulant solution are cerium, cesium, technetium, yttrium, zinc, uranium, and americium. Elements that show lower-than-desirable sorption from alkaline solution are strontium, chromium, cobalt, iron, manganese, zirconium, and plutonium.

6. Low-cost partitioning agents, such as Aliquat ${ }^{\mathrm{TM}} 336$, Cyanex ${ }^{\mathrm{TM}} 923$, and Reillex ${ }^{\mathrm{TM}} \mathrm{HPQ}$, often outperform other materials whose cost is hundreds of times higher.

7. Certain extractants, such as LIX-1010 and LIX-54, exhibit a large preference for americium over cerium and yttrium from basic solution, which indicates that a lanthanide/trivalent actinide group separation should be readily attainable. Elimination of lanthanides from the trivalent actinide fraction has been estimated to reduce the required number of glass logs by $5000,{ }^{5}$ which could save approximately $\$ 5$ billion.

8. Experimental anion exchange resins under development by LANL, referred to as JSK resins in this report, offer major advantages over the best previously known resins for removing plutonium from HLW media. An increased priority should be assigned to the development and commercial production of these resins.

9. Even the best partitioning agents for strontium provide only low-to-moderate Kd values, which may be inadequate for reliable separations under process conditions. Near-term efforts should focus on identifying or developing improved absorbers for strontium.

10. Iron, manganese, and chromium show low Kd values on most of the 60 absorbers evaluated. Because these elements are present at significant concentrations in many Hanford waste tanks, however, even low Kds could result in sufficiently high loadings of some absorbers to 
decrease their capacity for other elements. All Kds that we measured and report reflect realistic competition from these major matrix elements and therefore reflect what might be obtained with actual waste solution.

11. Measured Kd values for cobalt are low on all 60 absorbers from acidic solution and are only marginal from alkaline solution. If cobalt is to be removed from HLW solutions, other absorbers or extractants must be identified or developed.

12. Although elution was not a part of our study, the fact that we measured distributions from both acidic and basic solutions often indicates whether a change in acidity might elute a given element from a specific absorber.

13. SNL silico-titanate materials show outstanding sorption of cesium from acidic and basic simulant solutions. Because the sorption of cesium appears to be irreversible on silico-titanates, this matrix should be considered as a final waste form. Although there is concern that the high titanium content of these absorbers can easily exceed the assigned $1 \%$ limit for titanium in glass logs, the actual tolerance for titanium in glass (currently being measured) may be higher. Other waste forms, such as mineral analogues or different glass compositions, also may be compatible with silico-titanate absorbers. The excellent resistance of silico-titanates to loss of cesium by leaching may even allow this absorber to be safely incorporated in grout.

\section{ACKNOWLEDGMENTS}

This study was funded by the Hanford Program Office, EM-36, of the Office of Environmental Restoration and Waste Management, U S Department of Energy.

Clifford Mills of Group NMT-2, LANL, designed the tip-filter system that allowed disposable hypodermic syringes to be successfully used as container-dispensers during our many hundreds of dynamic contact experiments.

Frank McGarvey of Sybron Chemical Co. provided samples of macroporous anion exchange resins with trimethyl, triethyl, tripropyl, and tributyl amine functional groups.

Jane Bibler of SRS provided the sample of the SRS resorcinol/formaldehyde resin.

Dennis Fennelly of UOP Molecular Sieves provided samples of Ionsiv ${ }^{\text {TM }}$ TIE-96 for evaluation.

Elmer Klavetter and Larry Bustard of SNL provided samples of six silico-titanate absorbers and one hydrous titanium oxide absorber.

Ferdinand Sebesta, Department of Nuclear Chemistry, Czech Technical University, Prague, Czech Republic, prepared and provided samples of nine polyacrylonitrile-matrix composite absorbers for our evaluation.

Jerzy Narbutt, Department of Radiochemistry, Institute of Nuclear Chemistry and Technology, Warsaw, Poland, prepared and provided samples of two phenolsulfonic-formaldehyde-matrix composite absorbers for our evaluation. 
Stephen Yarbro and Gordon Jarvinen, Group NMT-2, LANL, provided valuable advice and suggestions during numerous technical discussions.

Stephen Agnew, Group INC-14, LANL, provided much detailed tank composition data, based on reconstruction from historical records, and many useful technical suggestions.

We especially appreciate the assistance and cooperation of many members of the Isotope and Nuclear Chemistry Division at Los Alamos National Laboratory, who are too numerous to mention individually.

\section{REFERENCES}

1. J. L. Ryan and E. J Wheelwright, "Recovery and Purification of Plutonium by Anion Exchange," Ind. Eng. Chem. 51(1), 60-65 (1959).

2. W. L. Poe, A. W. Joyce, and R. I. Martens, "Np-237 and Pu-238 Separation at the Savannah River Plant," I\&EC Proc. Design Devel. 3(4), 314-322 (1964).

3. J. A. Kelly, "Ion Exchange Process for Separating Americium and Curium from Irradiated Plutonium," Savannah River Laboratory report DP-1308 (1972).

4. S. L. Yarbro, W. A. Punjak, S. B. Schreiber, S. L. Dunn, G. D. Jarvinen, S. F. Marsh, S. F. Agnew, E. Birnbaum, and K. W. Thomas, "Status of Tank 102-SY Remediation Flowsheet Development at LANL," Los Alamos National Laboratory report LA-UR-93-1725 (April 1993).

5. J. L. Straalsund, J. L. Swanson, E. G. Baker, J. J. Holmes, E. O. Jones, and W. L. Kuhn, "Clean Option: An Alternative Strategy for Hanford Tank Waste Remediation: Volume 1. Overview," Pacific Northwest Laboratory report PNL-8388, Vol. 1 (December 1992).

6. R. K. Scheele and M. K. Peterson, "Results of the Characterization of Samples of Waste from Double-Shell Tank 102-SY," Westinghouse Hanford Company internal letter report (January 1990).

7. S. F. Agnew, "Preliminary Estimation of SY-102 Based on Tank History," Los Alamos National Laboratory internal letter report INC-14:93-sfal 645 (May 18, 1993).

8. G. J. Lumetta and J. L. Swanson, "Pretreatment of Plutonium Plant (PFP) Sludge: Report for the Period October 1990-March 1992," Pacific Northwest Laboratory report TWRSPP-92011 (June 9, 1992 draft).

9. R. Gunnink and J. B. Niday, "Computerized Quantitative Analysis by Gamma-Ray Spectrometry. Vol. 1. Description of the Gamanal Program," Lawrence Livermore Laboratory report UCRL-51061, Vol. 1 (March 1972). 
10. F. X. McGarvey and R. Gonzales, "Ion Exchange Studies on Strongly Basic Anion Exchange Resins Prepared with Tertiary Amines of Varying Molecular $\mathrm{W}^{\prime}$ ight," in Ion Exchange Advances, Proceedings of IEX '92, M. J. Slater, Ed. (Elsevier Science Publishing Ltd., Essex, England, 1992), pp. 97-103.

11. S. F. Marsh, "The Effect of Triethyl, Trimethyl, Tripropyl, and Tributyl Amine Functional Groups in Strong-Base Anion Exchange Resin on the Sorption of Pu(IV) from Nitric Acid, in Ion Exchange Processes: Advances and Applications, A. Dyer, M. J. Hudson, and P. A. Williams, Eds. (Royal Society of Chemistry, Cambridge, England, 1993), pp. 81-89.

12. F. Sebesta, A. Motl, and J. John, "Composite Ion-Exchangers, Their Development and Use," in Workshop of the Czech Technical University in Prague, INIS-mf-13201 or CONF-9201108 (January 1992), pp. 25-26.

13. J. Narbutt, A. Bilewicz, and B. Bartos, "Composite Ion Exchangers for Radiocaesium Removal from Nuclear Reactor Wastes," in Proc. Int. Symp. on Management of Low and Intermediate Level Radioactive Wastes, CONF-880510-, v-2 (Stockholm, May 16-20, 1988).

14. T. M. Suzuki, "Porous Spherical Resins Loaded with Hydrous Metal Oxides -Preparation and the Ion Exchange Properties," in New Developments in Ion Exchange; Materials, Fundamentals, and Applications, Proc. Int. Conf. on Ion Exchange, ICIE '91. M. Abe, Ed., (Elsevier, Amsterdam-Oxford-New York-Tokyo, 1992), pp. 181-186.

15. F. Sebesta and V. Stefula, "Composite Ion Exchanger with Ammonium Molybdophosphate and its Properties," J. Radioanalyt. Nucl. Chem., Articles, 140(1), 15-21 (1990).

16. S. F. Marsh and K. K. S. Pillay, "Effects of Ionizing Radiation on Modern Ion Exchange Materials," Los Alamos National Laboratory report LA-12655 (October 1993). 


\section{APPENDIX A}

\section{Preparation of Acid-Dissolved Sludge Simulant}

The composition for Hanford Tank 102-SY sludge was provided by Westinghouse Hanford Company (WHC) personnel in letter 7K321-92-RAK-02. However, aside from the $0.6 \mathrm{M}$ phosphate value in the single tank sample cited by WHC, no other 102-SY sample contained more than $0.11 \mathrm{M}$ phosphate, and the phosphate level of most samples was near $0.05 \mathrm{M}$. For these reasons, we adjusted the initial phosphate level from the WHC-recommended value of $0.6 \mathrm{M}$ to $0.1 \mathrm{M}$.

We further adjusted the sludge composition to reflect partial dissolution during the planned mixing of HLW Tank 102-SY supernate and solids during retrieval operations.

Lumetta and Swanson ${ }^{8}$ estimated that dilute caustic washing would remove $69 \%$ of the initial $\mathrm{Al}, 17 \%$ of the initial $\mathrm{Cr}$, and $10 \%$ of the initial $\mathrm{Pb}$. Lumetta also agreed with our decision to change the initial phosphate level to $0.1 \mathrm{M}$ and estimated that washing the sludge with dilute caustic solution would leach out $90 \%$ of the phosphate, $90 \%$ of the chloride, $90 \%$ of the sulfate, and $75 \%$ of the fluoride.

Using these figures as guidelines, we recalculated the composition of Tank 102-SY sludge, as it would exist after washing with the alkaline supernate solution. We then prepared the recalculated simulant composition in a minimum concentration of nitric acid to represent caustic-washed, acid-dissolved sludge. Lumetta agreed that this would represent the most realistic simulant of the feed for a partitioning process.

Table A. Composition of Acid-Dissolved Sludge Simulant

\begin{tabular}{|c|c|c|c|}
\hline Component & Initial Conc., $M$ & Factor & Adjusted Conc., M \\
\hline $\mathrm{Al}$ & 1.65 & 0.31 & 0.51 \\
\hline $\mathrm{Ca}$ & 0.16 & 1.0 & 0.16 \\
\hline $\mathrm{Cr}$ & ().48 & 0.83 & 0.40 \\
\hline $\mathrm{Cu}$ & 0.02 & 1.0 & $0 .(1) 2$ \\
\hline $\mathrm{Fe}$ & 0.45 & 1.0 & 0.45 \\
\hline $\mathrm{K}$ & 0.08 & 0.10 & 0.008 \\
\hline $\mathrm{Mg}$ & 0.07 & 1.0 & 0.07 \\
\hline $\mathrm{Mn}$ & 0.165 & 1.0 & 0.165 \\
\hline $\mathrm{Na}$ & 6.0 & (). 10 & ().60) \\
\hline $\mathrm{Ni}$ & 0.01 & 1.0 & $(0.01$ \\
\hline $\mathrm{Pb}$ & 0.016 & 0.9() & $(0.014$ \\
\hline $\mathrm{Se}$ & 0.03 & 1.0 & 0.0 .3 \\
\hline $\mathrm{Si}$ & 0.08 & 1.0 & 0.08 \\
\hline $\mathrm{Sr}$ & 0.001 & 1.0 & $0.0(0) 1$ \\
\hline Th & 0.0 .3 & 1.0 & 0.03 \\
\hline $\mathrm{U}$ & 0.03 & 1.0 & 0.0 .3 \\
\hline $\mathrm{Cl}$ & 0.15 & 0.10 & 0.015 \\
\hline $\mathrm{F}$ & 0.30 & 0.25 & 0.07 .5 \\
\hline $\mathrm{PO}_{4}$ & 0.10 & 0.10 & 0.01 \\
\hline $\mathrm{SO}_{4}^{4}$ & (0.10 & (). 10 & $0 .(0) 1$ \\
\hline
\end{tabular}


To the composition shown in Table A, plutonium was added as ${ }^{231} \mathrm{Pu}$ to obtain $3 \mathrm{~g} \mathrm{Pu/L}$; in a few cases ${ }^{238} \mathrm{Pu}$ was added, instead, to obtain $60 \mathrm{mg} \mathrm{Pu/L}$.

We assumed that all hydroxide would be neutralized and that all carbonate would decompose when the sludge is dissolved in acid.

\section{PROCEDURE}

The following components were combined:

$\begin{array}{cllc}\text { Component } & \text { Compound taken } & \text { Moles } & \text { Grams } \\ & & & \\ \mathrm{Ca} & \mathrm{Ca}\left(\mathrm{NO}_{3}\right)_{2} \cdot 4 \mathrm{H}_{2} \mathrm{O} & 0.16 & 37.8 \\ \mathrm{Cr} & \mathrm{Cr}\left(\mathrm{NO}_{3}\right)_{3} \cdot 9 \mathrm{H}_{2} \mathrm{O} & 0.40 & 160 \\ \mathrm{Cu} & \mathrm{Cu}\left(\mathrm{NO}_{3}\right)_{2} \cdot 2.5 \mathrm{H}_{2} \mathrm{O} & 0.02 & 4.65 \\ \mathrm{Fe} & \mathrm{Fe}\left(\mathrm{NO}_{3}\right)_{3} \cdot 9 \mathrm{H}_{2} \mathrm{O} & 0.45 & 181.8 \\ \mathrm{~K} & \mathrm{KNO}_{3} & 0.008 & 0.81 \\ \mathrm{Mg} & \mathrm{Mg}\left(\mathrm{NO}_{3}\right)_{2} \cdot 6 \mathrm{H}_{2} \mathrm{O} & 0.07 & 17.9 \\ \mathrm{Mn} & \mathrm{Mn}\left(\mathrm{NO}_{3}\right)_{2} & 0.165 & 57 \mathrm{ml} 51 \% \text { scilution } \\ \mathrm{Na} & \mathrm{NaNO}_{3} & 0.44 & 37.4 \\ \mathrm{Ni} & \mathrm{Ni}\left(\mathrm{NO}_{3}\right)_{2} \cdot 6 \mathrm{H}_{2} \mathrm{O} & 0.01 & 2.91 \\ \mathrm{~Pb} & \mathrm{~Pb}\left(\mathrm{NO}_{3}\right)_{2} & 0.014 & 4.63 \\ \mathrm{Sr} & \mathrm{Sr}\left(\mathrm{NO}_{3}\right)_{2} & 0.001 & 0.21\end{array}$

Then 0.51 mole of aluminum was added as $230 \mathrm{ml}$ of $2.2 \mathrm{M} \mathrm{Al}\left(\mathrm{NO}_{3}\right)_{3}$ solution, swirled to mix, and allowed to sit overnight. The liquid portion, about $1 / 3$ of the total, was decanted into a separate container. Then $100 \mathrm{ml}$ of $0.5 \mathrm{M}$ nitric acid was added to the solids and the mixture was warmed in a hot-water bath. Most of the solids dissolved. The remaining solids dissolved in another $100-\mathrm{ml}$ portion of $0.5 \mathrm{M}$ nitric acid.

Thorium was added to the solution:

Th

$\mathrm{Th}\left(\mathrm{NO}_{3}\right)_{4} \cdot 5 \mathrm{H}_{2} \mathrm{O}$

0.03

17.1

An appropriate portion of $\mathrm{UO}_{2}$ was dissolved in $40 \mathrm{ml}$ of water and $4 \mathrm{ml}$ of concentrated nitric acid, and was added to the solution.
$\mathrm{U}$
$\mathrm{UO}_{2}$
0.03
8.1

The other anions were added as portions of the appropriate acid to $40 \mathrm{ml}$ of water, which then was added to the stirred solution.

$\begin{array}{llll}\mathrm{Cl} & \mathrm{HCl} & 0.015 & 1.3 \mathrm{ml} \text { conc. acid } \\ \mathrm{PO}_{4} & \mathrm{H}_{3} \mathrm{PO}_{4} & 0.01 & 0.7 \mathrm{ml} \text { conc. acid } \\ \mathrm{SO}_{4} & \mathrm{H}_{2} \mathrm{SO}_{4} & 0.01 & 0.6 \mathrm{ml} \text { conc. acid }\end{array}$


Sodium silicate was dissolved in $50 \mathrm{ml}$ of warm water.

$\mathrm{Si}$

$\mathrm{Na}_{2} \mathrm{SiO}_{3} \cdot 9 \mathrm{H}_{2} \mathrm{O}$

22.7

To this silicate solution, $2.6 \mathrm{ml}(0.075$ mole $)$ of concentrated HF was added.

The silicate-fluoride solution was mixed well and added, with stirring, to the total solution. A precipitate formed immediately, but seemed to disperse as the solution was stirred and warmed in a hot-water bath.

Another $50 \mathrm{ml}$ of $0.5 \mathrm{M}$ nitric acid was added and stirred while the solution was being warmed.

Finally, 0.03 mole of selenium was added as 3.87 grams of $\mathrm{H}_{2} \mathrm{SeO}_{3}$, which was dissolved directly in the simulant solution. After cooling to room temperature, sufficient $0.5 \mathrm{M}$ nitric acid was added to bring the solution volume to 1.00 liter.

Silica, which precipitated as expected, was removed by filtration after the simulant preparation was complete. We therefore recommend that future efforts to prepare acid-dissolved sludge simulants omit the silicate component, as it only creates a messy precipitate that must be removed. 


\section{APPENDIX B \\ Preparation of Simulated Alkaline Supernate Solution}

The composition data for the supernate solution of Hanford Tank 102-SY was provided by Stephen Agnew of Group INC-14 at LANL, who hased the composition on tank records and analytical results. ${ }^{7}$ The supernate composition was adjusted to what would be expected after the tank contents were thoroughly mixed, that is, after thorough washing of the sludge with the alkaline supernate solution. This is appropriate because we took credit for what would be dissolved during sludge washing when we estimated the composition of the caustic-washed, aciddissolved sludge simulant.

The highest concentration was used whenever there was a range of uncertainty for a given component, which resulted in an insoluble fraction that was removed by filtration. The fact that some portion of the metal ions remained undissolved even after we stirred the mixture continuously for $40 \mathrm{~h}$ assured us that the solution was saturated, as the actual supernate solution would be after sludge washing.

All components calculated to be present at a concentration of $0.01 \mathrm{M}$ or higher are included in this simulated supernate solution.

We recognize that our best composition estimate does not provide an ionic balance. Our supernate solution therefore has significantly less sodium, more nitrate, and somewhat more chloride than the targeted amounts.

Table B. Composition of Simulated Alkaline Supernate Solution

\begin{tabular}{|c|c|c|}
\hline Component & Target Conc., $\mathbf{M}$ & Actual Conc., $\mathbf{M}$ \\
\hline $\mathrm{Al}$ & 0.42 & 0.42 \\
\hline $\mathrm{Ca}$ & ().2.3 & (1).012.3 \\
\hline $\operatorname{Cr}($ III $)$ & 0.074 & $(0.074$ \\
\hline $\mathrm{Cr}(\mathrm{VI})$ & $0.0) 72$ & $0 .(1) 72$ \\
\hline $\mathrm{Fe}$ & 0.097 & 0.097 \\
\hline $\mathrm{K}$ & 0.078 & 0.078 \\
\hline $\mathrm{Mg}$ & 0.011 & 0.011 \\
\hline $\mathrm{Mn}$ & 0.038 & 0.0 .38 \\
\hline $\mathrm{Na}$ & 2.34 & 1.39 \\
\hline $\mathrm{Si}$ & $(0.019$ & $(0.019$ \\
\hline $\mathrm{Cl}$ & 0.024 & 0.046 \\
\hline $\mathrm{F}$ & 0.107 & 0.107 \\
\hline $\mathrm{NO}_{3}$ & 0.867 & 1.87 \\
\hline $\mathrm{NO}_{2}^{-}$ & 0.193 & 0.193 \\
\hline $\mathrm{PO}_{4}^{3}$ & 0.12 & 0.12 \\
\hline $\mathrm{SO}_{4}^{2-2}$ & 0.023 & 0.023 \\
\hline $\mathrm{CO}_{3}^{2-}$ & 0.11 & 0.11 \\
\hline $\mathrm{OH}^{\prime}$ & 0.54 & 0.54 \\
\hline Density & 1.27 & 1.21 \\
\hline
\end{tabular}


To the composition shown in Table $\mathrm{B},{ }^{238} \mathrm{Pu}\left(\mathrm{CO}_{3}\right)_{2}$ wals added to obtain $60 \mathrm{mg} \mathrm{Pu} / \mathrm{L}$.

\section{PROCEDURE}

The following components were combined:

\begin{tabular}{|c|c|c|c|}
\hline Component & Compound taken & Moles & Grams \\
\hline $\mathrm{Ca}$ & $\mathrm{CaCl}_{2}$ & 0.023 & 2.55 \\
\hline $\mathrm{Cr}$ & $\mathrm{Cr}\left(\mathrm{NO}_{3}\right)_{3} \cdot 9 \mathrm{H}_{2} \mathrm{O}$ & 0.074 & 29.6 \\
\hline $\mathrm{Cr}(\mathrm{VI})$ & $\mathrm{K}, \mathrm{Cr}, \mathrm{O}$ & 0.0072 & 2.12 \\
\hline $\mathrm{Fe}$ & $\mathrm{Fe}\left(\mathrm{NO}_{3}\right)_{3} \cdot 9 \mathrm{H}_{2} \mathrm{O}$ & 0.097 & 39.2 \\
\hline K & $\mathrm{KOH}$ & 0.078 & 4.37 \\
\hline $\mathrm{Mg}$ & $\mathrm{Mg}\left(\mathrm{NO}_{3}\right)_{2} \cdot 6 \mathrm{H}_{2} \mathrm{O}$ & 0.011 & 2.81 \\
\hline $\mathrm{Mn}$ & $\mathrm{Mn}\left(\mathrm{NO}_{3}\right)_{2}$ & 0.038 & $13.3 \mathrm{~g} 51 \%$ solution \\
\hline
\end{tabular}

$\mathrm{Al}\left(\mathrm{NO}_{3}\right)_{3}$ was added as $190 \mathrm{ml}$ of $2.2 \mathrm{M}$ solution and mixed by swirling.
$\mathrm{Al}$
$\mathrm{Al}\left(\mathrm{NO}_{3}\right)_{3}$ solution
0.42
$190 \mathrm{ml}$

$\mathrm{NaOH}$ was added as $50 \%$ solution.

$\mathrm{Na}$

$\mathrm{NaOH}$ soln.

0.538

$43 \mathrm{~g}$ of $50 \%$ solution.

Miost other anions then were added as the appropriate sodium salts.

$\begin{array}{llcc}\mathrm{Cl} & \text { (added above as } \mathrm{CaCl}_{2} \text { ) } & & \\ \mathrm{F} & \mathrm{NaHF}_{2} & 0.107 & 3.32 \\ \mathrm{PO}_{4} & \mathrm{Na}_{2} \mathrm{HPO}_{4} \cdot 7 \mathrm{H}_{2} \mathrm{O} & 0.061 & 16.4 \\ \mathrm{NO}_{3} & \text { (added above as many salts) } & & \\ \mathrm{NO}_{2} & \mathrm{NaNO}_{2} & 0.193 & 16.5 \\ \mathrm{SO}_{4} & \mathrm{Na}_{2} \mathrm{SO}_{4} & 0.023 & 3.27 \\ \mathrm{CO}_{3} & \mathrm{Na}_{2} \mathrm{CO}_{3} \cdot \mathrm{H}_{2} \mathrm{O} & 0.111 & 13.8\end{array}$

Finally, sodium silicate was added.
$\mathrm{Si}$
$\mathrm{Na}_{2} \mathrm{SiO}_{3} \cdot 9 \mathrm{H}_{2} \mathrm{O}$
0.08
22.7

The solution was diluted with water to nearly I liter and stirred continuously on a magnetic mixer for $40 \mathrm{~h}$. Because a large amount of solids remained undissolved, the solution was centrifuged to separate most of the solids and then filtered. The settled undissolved solids occupied approximately $25 \mathrm{cc}$. After filtration, the solution was diluted to 1.00 liter with water.

The density of 1.21 for the filtered solution was somewhat lower than the density of 1.27 calculated by Stephen Agnew, which we attribute to the undissolved (and removed) solids. 


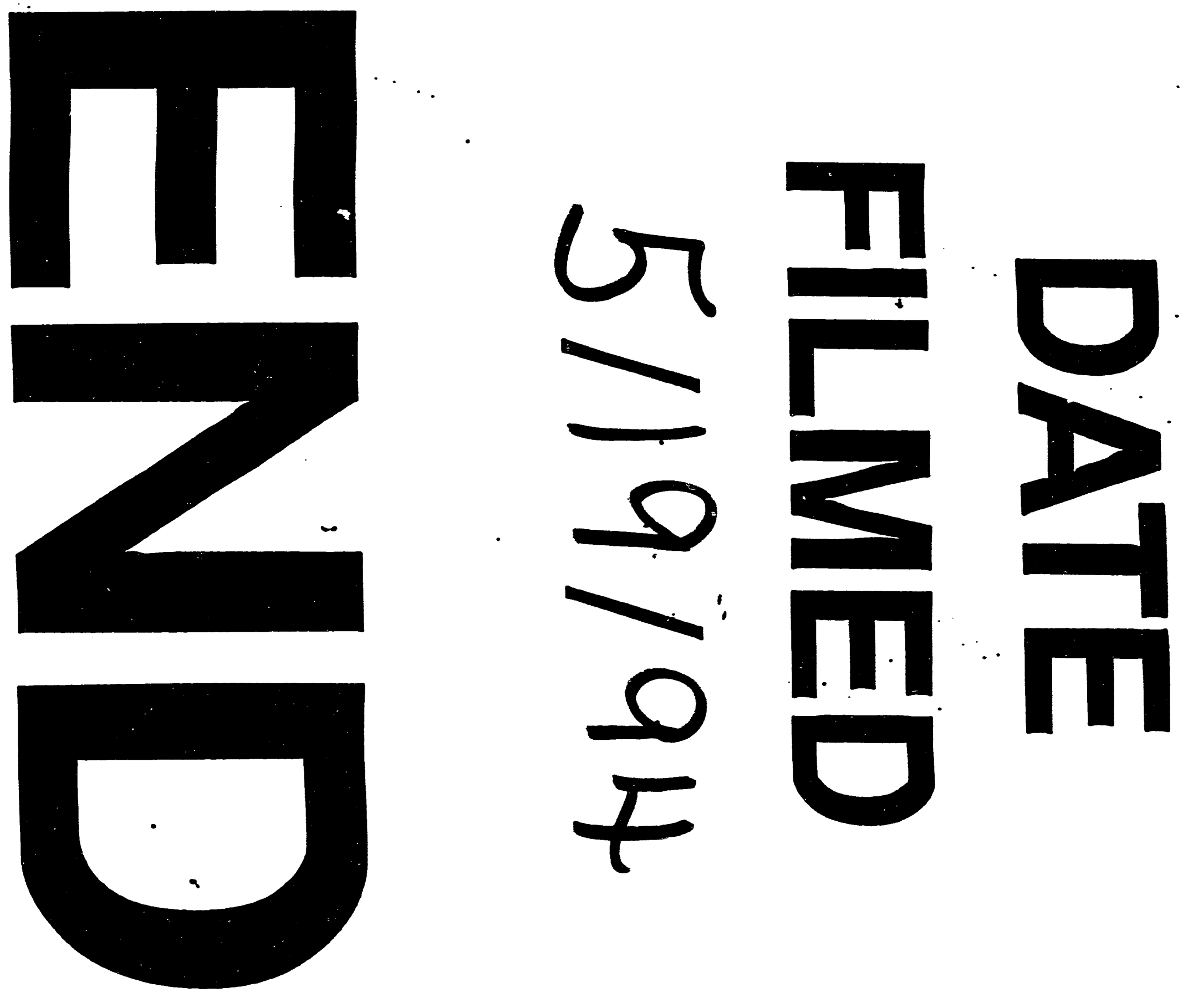


